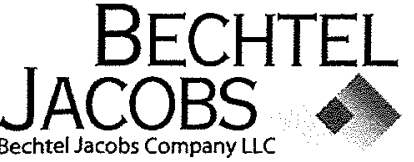

DOE Contract No. DE-AC05-98OR22700

Job No. 23900

TD-2009-0049

November 5, 2009

Mr. Jason Darby

EMWMF Program Manager

U. S. Department of Energy

Oak Ridge Operations Office

P. O. Box 2001

Oak Ridge, Tennessee 37830

Dear Mr. Darby:

Transmittal of the Calculation Package that supports the Analysis of Performance of the Environmental Management Waste Management Facility Oak Ridge, Tennessee (Based 5-cell design issued 8/14/09)

Enclosed is the calculation package that supports the Analysis of Performance of the Environmental Management Waste Management Facility (EMWMF), Oak Ridge, Tennessee (Based 5-cell design issued 8/14/09) for transmission to the members of the EMWMF Core Team.

If you have any questions regarding this transmittal, please contact me at 241-1268.

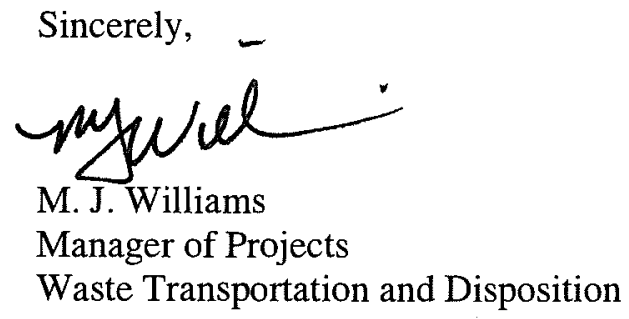

MJW:mll

Enclosure: As stated

c/enc: ORO EM Mailroom

File-DMC-RC 


\section{Calculation Package \\ for the \\ Analysis of Performance of the \\ Environmental Management Waste Management Facility \\ Oak Ridge, Tennessee \\ (Based 5-cell design issued 8/14/09)}

Date Issued: September 14, 2009

This document is approved for public

release per reviaw by:

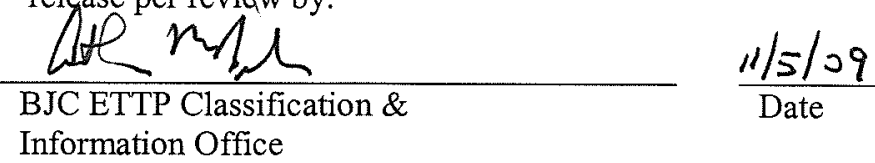




\title{
Calculation Package for the Analysis of Performance of the Environmental Management Waste Management Facility Oak Ridge, Tennessee (Based 5-cell design issued 8/14/09)
}

Date Issued: September 14, 2009

\author{
Prepared by \\ Jacobs Engineering Group Inc. \\ Oak Ridge, Tennessee \\ and \\ Portage Environmental Inc. \\ Salt Lake City, Utah \\ Prepared for \\ the \\ U.S. Department of Energy \\ Office of Environmental Management \\ BECHTEL JACOBS COMPANY LLC \\ managing the \\ Environmental Management Activities at the \\ East Tennessee Technology Park \\ Y-12 National Security Complex Oak Ridge National Laboratory \\ under contract DE-AC05-98OR22700 \\ for the
}

\section{U.S. DEPARTMENT OF ENERGY}




\section{INTRODUCTION}

This document presents the results of an assessment of the performance of a build-out of the Environmental Management Waste Management Facility (EMWMF). The EMWMF configuration that was assessed includes the as-constructed Cells 1 through 4 , with a groundwater underdrain that was installed beneath Cell 3 during the winter of 2003-2004, and Cell 5, whose proposed design is an Addendum to Remedial Design Report for the Disposal of Oak Ridge Reservation Comprehensive Environmental Response, Compensation, and Liability Act of 1980 Waste, Oak Ridge, Tennessee, DOE/OR/01-1873\&D2/A5/R1.

The total capacity of the EMWMF with 5 cells is about 1.7 million cubic yards. This assessment was conducted to determine the conditions under which the approved Waste Acceptance Criteria (WAC) for the EMWMF found in the Attainment Plan for Risk/Toxicity-Based Waste Acceptance Criteria at the Oak Ridge Reservation, Oak Ridge, Tennessee [U.S. Department of Energy (DOE) 2001a], as revised for constituents added up to October 2008, would remain protective of public health and safety for a five-cell disposal facility. For consistency, the methods of analyses and the exposure scenario used to predict the performance of a five-cell disposal facility were identical to those used in the Remedial Investigation and Feasibility Study (RI/FS) and its addendum (DOE 1998a, DOE 1998b) to develop the approved WAC. To take advantage of new information and design changes departing from the conceptual design, the modeling domain and model calibration were updated from those used in the RI/FS and its addendum. It should be noted that this analysis is not intended to justify or propose a change in the approved WAC.

\section{EXPOSURE SCENARIO}

The exposure scenario used in the assessment consists of a resident farmer located near the confluence of Bear Creek and Northern Tributary-5 (NT-5). Bear Creek surface water is used for watering livestock and irrigating crops, and drinking water is obtained from a well located near NT-5, between the EMWMF and Bear Creek. This exposure scenario is identical to the exposure scenario used to develop the approved WAC. Figure 1 shows the relative locations of the well, five-cell EMWMF, and Bear Creek and its tributaries used in the RL/FS and addendum analyses.

\section{REVISION OF THE MODEL DOMAIN}

The RI/FS performance modeling domain and the larger modeling domain used in this analysis are also shown in Figure 1. Because the EMWMF is being constructed further to the north and east of the conceptual location of the EMWMF, the modeling domain was revised to analyze the EMWMF in its present location. Detailed descriptions of the groundwater modeling domain and associated specific features are given in Appendix B of the Engineering Feasibility Plan for Groundwater Suppression at the Environmental Management Waste Management Facility, Oak Ridge, Tennessee [Bechtel Jacobs Company LLC] (BJC 2003). Essential features of the revised modeling domain are: 
- The refined model grid is extended north to the top of Pine Ridge (a zero flow groundwater modeling boundary);

- The western groundwater hydraulic head boundary is moved from its previous location in proximity to NT-5 to the west, past NT-6.

Additional changes in the modeling that could influence the projected performance of the EMWMF with an underdrain include:

- A change in the cover design that reduced the rate of infiltration through the waste (DOE 2001b);

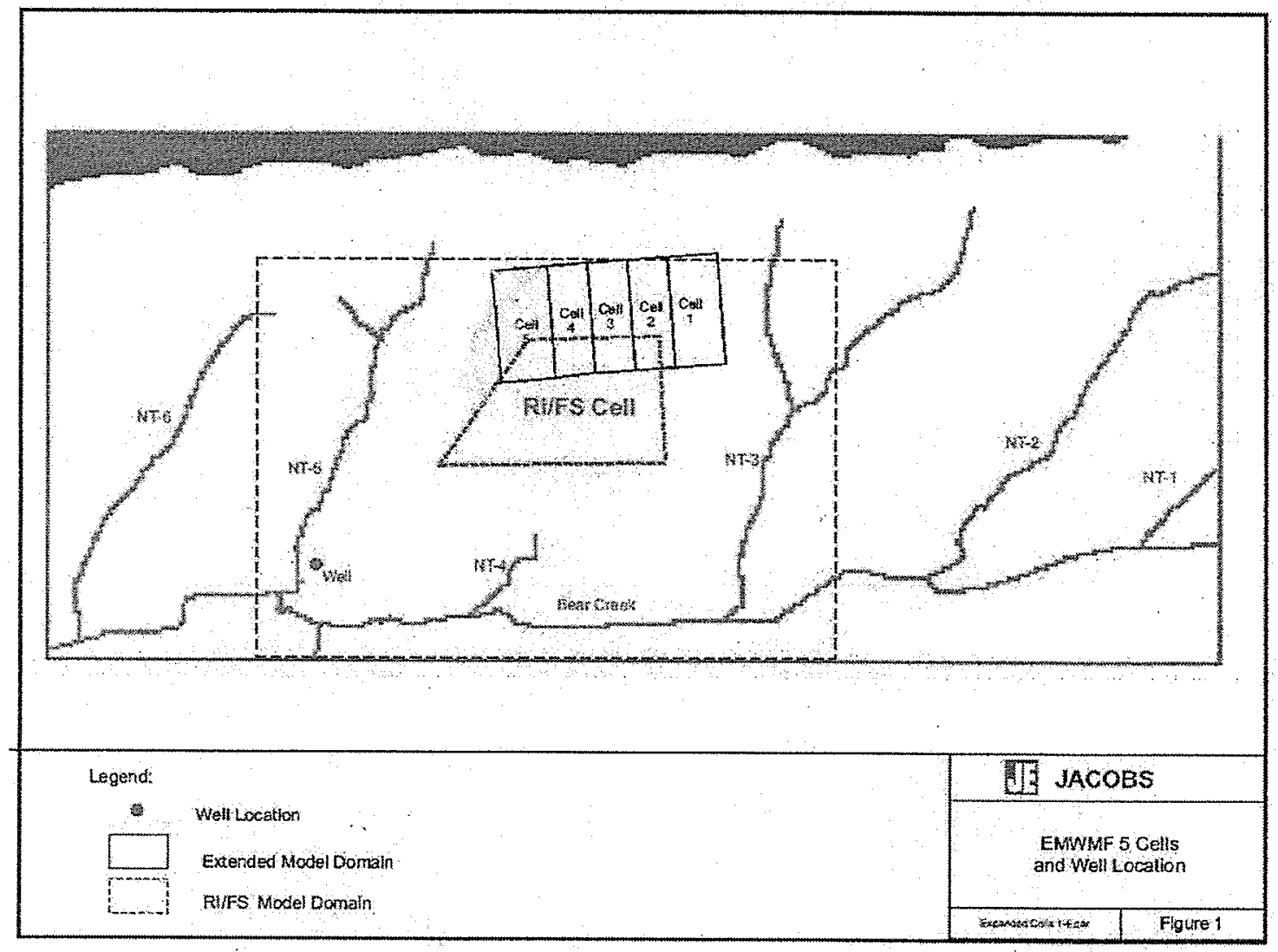

Figure 1. Original modeling domain and the modeling domain used in this analysis, and locations of RI/FS and Addendum disposal facility, EMWMF Cells 1 through 5, and receptor well.

- The addition of an underdrain beneath Cell 3 that was installed in late 2003 and early 2004 (DOE 2003);

- An increase in the total plan area of the EMWMF; and 
- Calibration of the groundwater model to newly acquired groundwater and geological data (BJC 2003).

\section{MODELING CONTAMINANT MIGRATION}

The following analyses were performed under the assumption that Cells 1 through 5 have been closed and capped:

- The groundwater flow fields were assessed using the MODFLOW groundwater model and the revised modeling domain, adjusted for the proposed build-out of the EMWMF (Cells 1 through 5) and location, including the underdrain. Details of these revisions, the resulting groundwater characteristics, and flow calibration are available in BJC 2003.

- Solute transport calculations were made for movement of contaminants from the disposal facility to the residential well using MT3D.

- Dilution factors (DFs) for Bear Creek and the residential well were calculated using the revised modeling domain for the EMWMF (Cells 1 through 5) with an underdrain beneath Cell 3. The DFs are defined as the ratios of the peak steady-state contaminant concentrations in the Bear Creek water or water continuously pumped ( 250 gallons per day) from the well to unit contaminant concentrations entering the groundwater beneath the disposal facility.

\section{METHOD OF RISK AND DOSE ASSESSMENT}

The projected peak risks and doses in the first 100,000 years after closure from radioactive or hazardous constituents were calculated for consumption of drinking water from a well and using Bear Creek surface water for agricultural purposes with a concentration of contaminants in the waste (source term) of $1 \mathrm{Ci} / \mathrm{m}^{3}$ (curie per cubic meter) for radioactive constituents and $1 \mathrm{~kg} / \mathrm{m}^{3}$ (kilogram per cubic meter) for toxicological constituents, respectively. Those risks and doses were scaled according to the approved WAC concentrations to determine the risks and doses that could occur if each constituent was present alone in the EMWMF at the WAC concentration. Those risks and doses were then compared to the risk and dose criteria upon which the WAC are based.

The calculations were performed in the following steps:

1. PATHRAE [Rogers and Associates Engineering (RAE) 1995a and RAE 1995b] calculations were performed to determine the equivalent annual water consumption per year for Bear Creek [defined as the Equivalent Uptake (EU)]. This equivalent water consumption corresponds to scaling the use of Bear Creek water for drinking and agricultural purposes to an equivalent annual drinking water ingestion that would give the same annual constituent uptake as calculated to come from all pathways. Since drinking water in the resident farmer exposure scenario will be supplied by a well rather than Bear Creek, the annual drinking water volume of $7301 / \mathrm{yr}$ to be supplied by the well is subtracted from the Bear Creek EU to estimate the effective drinking water ingestion that can be associated with agricultural uses for Bear Creek surface water. The PATHRAE calculations also provide peak concentrations 
of contaminants in Bear Creek water corresponding to a unit source term, the corresponding peak doses or risks associated with those concentrations, and the times of occurrence of the peak concentrations.

2. The calculated DFs for Bear Creek and the residential well were used for scaling the constituent concentrations in Bear Creek to corresponding well concentrations. Using the build-out of the EMWMF disposal cell configuration and design, the ratio of the constituent concentrations in the well to those in Bear Creek is $\mathrm{DF}_{\mathrm{Well}_{1}} / \mathrm{DF}_{\text {Creek }}=0.00060 / 0.0035=0.17$.

3. The peak effective risk or dose was calculated as the risk or dose due to ingestion of $730 \mathrm{l} / \mathrm{yr}$ per year of water drawn from the well, plus the consumption of agricultural products and livestock irrigated or watered with Bear Creek surface water. The latter is calculated by subtracting $7301 / \mathrm{yr}$ of water from the EU for Bear Creek water that is calculated by the PATHRAE computer code. Thus:

Peak Effective Risk or Dose $=$ Peak Bear Creek Risk or Dose $\mathrm{x}$

$$
\left[\mathrm{EU}-730+\left(\mathrm{DF}_{\mathrm{Well}} / \mathrm{DF}_{\text {Creek }}\right) \times 730\right] / \mathrm{EU}
$$

where $\mathrm{DF}_{\mathrm{Well}}$ and $\mathrm{DF}_{\text {Creek }}$ are the dilution factors calculated for the well and the creek, respectively, and the peak Bear Creek risk or dose correspond to ingestion of Bear Creek water at the annual EU rate.

\section{$\underline{\text { Radioactive Constituents - Risk }}$}

The Peak Bear Creek Risk for radioactive constituents is,

Peak Bear Creek Risk $=$ Peak Bear Creek concentration x EU x

Slope Factor $\mathrm{x} 30$ years exposure duration,

where the peak Bear Creek risk is calculated directly by the PATHRAE-RAD computer code using slope factors [Incremental Lifetime Cancer Risk (ILCR)/pCi] obtained from the EPA's Health Effects Assessment Summary Tables.

Hazardous Constituents - Risk and Dose

Peak Bear Creek Risk $=$ Peak Bear Creek Lifetime Intake of Carcinogens $\mathrm{x}$ Slope Factor,

Peak Bear Creek Lifetime Intake for Carcinogens $=$

Peak Bear Creek Concentration x

EU x 30 years exposure duration / 
and,

[70 kg body weight x 365 days per year x 70 year life]

Peak Bear Creek Daily Intake (Dose) for Non-Carcinogens =

Peak Bear Creek Concentration x EU /

[70 kg body weight $\mathrm{x} 365$ days per year],

where the peak Bear Creek daily intake for carcinogens is calculated using PATHRAE-HAZ generated data and the formula immediately above.

The peak risks and doses resulting from constituent concentrations in the waste equal to the WAC concentrations were then calculated as follows:

For radioactive constituents:

Peak Effective Risk for constituents in the waste at the WAC level =

[Peak Effective Risk from a $1 \mathrm{Ci} / \mathrm{m}^{3}$ source $\mathrm{x}$ WAC] $/ 6.25 \times 10^{5}$

where the WAC are expressed in picuries per gram $(\mathrm{pCi} / \mathrm{g})$ and the factor $6.25 \times 10^{5}$ results from unit conversions.

For hazardous constituents:

Peak Effective Risk or Dose for constituents in the waste at the WAC level =

[Peak Effective Risk or Dose from a $1 \mathrm{~kg} / \mathrm{m}^{3}$ source $\mathrm{x}$ WAC] $/ 625$

where the WAC are expressed in milligrams per kilogram $(\mathrm{mg} / \mathrm{kg})$ and the factor of 625 comes from unit conversions.

\section{MODELING RESULTS FOR THE EMWMF CELLS 1 THROUGH 5 WITH UNDERDRAIN}

Peak risk and dose calculations for the EMWMF Cells 1 through 5 with an underdrain using the PATHRAE-RAD and PATHRAE-HAZ environmental pathway analysis computer codes are based on the following input parameters and data, some of which were generated using MODFLOW and MT3D for the extended modeling domain.

Volume-weighted average waste height $50.2 \mathrm{ft}=15.3 \mathrm{~m}$ 
Effective Cells $1-5$ horizontal dimensions $=1,722 \mathrm{ft} \times 545 \mathrm{ft}=525 \mathrm{~m} \times 166 \mathrm{~m}$

Waste footprint area $=938,490 \mathrm{ft}^{2}=87,150 \mathrm{~m}^{2}$

Waste volume $=47.1 \times 10^{6} \mathrm{ft}^{3}=1.74 \times 10^{6} \mathrm{cy}=1.3 \times 10^{6} \mathrm{~m}^{3}$

Infiltration rate $=0.36$ inches $/$ year $=0.91 \mathrm{~cm} /$ year

Volume-weighted average distance to Bear Creek or to a drainage feature that gives quick access to Bear Creek $=242 \mathrm{ft}=73.9 \mathrm{~m}$

Creek dilution factor $\left(\mathrm{DF}_{\text {Creek }}\right)=3.5 \times 10^{-3}$

Well dilution factor $\left(\mathrm{DF}_{\text {Well }}\right)=6.0 \times 10^{-4}$

The peak risks and doses calculated using the PATHRAE code for Cells 1 through 5 with an underdrain beneath Cell 3, based on unit source terms, are given in Tables 1 and 2 for the radioactive and hazardous constituents, respectively. The PATHRAE input parameters used in these calculations and summaries of output results are given in Attachment 1. Note that the peak doses in Bear Creek in Table 2, as given by PATHRAE, are always higher than their corresponding peak effective doses because the former are calculated based on the assumption that Beak Creek water is used for all purposes. The constituent concentrations in the well are lower than in Bear Creek and, since most of the effective uptake for most constituents is from drinking water (i.e., water from the well), the effective dose is usually considerably lower.

The projected peak risks and doses from the proposed 5-cell EMWMF with the underdrain using the approved WAC as the source terms, and the corresponding criteria used in developing the approved WAC, are given in Tables 3 and 4.

\section{CONCLUSION}

If the approved list of WAC constituents that have finite WAC values (given in Revised Table A.1: Analytic WAC Limits, http://bechteljacobs.org/webindex.html\#3) are assumed to be in the EMWMF at the maximum allowable concentrations prescribed by their respective WAC, excluding cabazole (see below), the resultant risks and doses to the receptor are summarized in Tables 3 and 4. It can be seen that those risks and doses will not exceed the current WAC criteria for all the constituents.

The analyses that led to the development of the projected WAC in the Table A.1 showed that, for the original 4-cell configuration of the EMWMF, the concentrations of constituents in the creek water and the well water would be approximately the same. Those analyses also demonstrated that, for the vast majority of the constituents, the most of the risks and doses to the receptor comes from drinking well water. Any major reduction in constituent concentrations in the groundwater at the well will greatly reduce projected risks and doses. Groundwater modeling shows that the subsequent addition of an underdrain beneath Cell 3 that diverts much of the 
leachate directly to Bear Creek via the remnant of NT-4, as well as the extension of the EMWMF westward, significantly reduces constituent concentrations in the well water.

In doing the calculations for the revised 5-cell EMWMF, all of the WAC in Table A.1 were used except for the WAC for carbazole. It has been recognized that the WAC given for carbazole in Table A.1 is probably too high because the $\mathrm{K}_{d}$ used in its calculation was too high. Consequently, a preliminary revised WAC for carbazole of 5.40E+4 was calculated for the 4-cell EMWMF configuration without an underdrain, employing the data sources and protocols that are presently used to calculate WACs for newly found constituents. This scenario was analyzed to establish the" baseline" WAC for carbazole associated with the original 4-cell design so that it might be used for comparison purposes to current 5-cell design. That carbazole WAC was used in determining projected peak risk shown in Table 4 for that constituent for the current 5 -cell design which is below the risk criteria of 1.0E-04. 
Table 1. Peak effective risks for the EMWMF for radioactive constituents (risks based on a $1 \mathrm{Ci} / \mathrm{m}^{3}$ concentration in the waste)

\begin{tabular}{|c|c|c|c|c|c|}
\hline Nuclide & $\begin{array}{c}\text { Peak } \\
\text { Concentration in } \\
\text { Bear Creek } \\
(\mathrm{pCi} / \mathrm{L})\end{array}$ & $\begin{array}{c}\text { Ingestion } \\
\text { Slope Factor } \\
(1 / \mathrm{pCi})\end{array}$ & $\begin{array}{l}\text { Equivalent } \\
\text { Uptake } \\
\text { (L/yr) }\end{array}$ & $\begin{array}{c}\text { Peak } \\
\text { Effective } \\
\text { Risk } \\
\text { (ILCR) }\end{array}$ & $\begin{array}{l}\text { Time of } \\
\text { Projected } \\
\text { Peak } \\
\text { (yr) }\end{array}$ \\
\hline $\mathrm{H}-3$ & $1.54 \mathrm{E}+01$ & $7.15 \mathrm{E}-14$ & $1.17 \mathrm{E}+03$ & $2.18 \mathrm{E}-08$ & 220 \\
\hline $\mathrm{C}-14$ & $1.71 E+06$ & $1.03 \mathrm{E}-12$ & $9.56 \mathrm{E}+02$ & $2.12 \mathrm{E}-02$ & 270 \\
\hline TC-99 & $1.52 \mathrm{E}+06$ & $1.40 \mathrm{E}-12$ & $7.40 \mathrm{E}+02$ & $1.16 \mathrm{E}-02$ & 300 \\
\hline I-129 & $7.94 \mathrm{E}+05$ & $1.84 \mathrm{E}-10$ & $8.33 \mathrm{E}+02$ & $1.19 \mathrm{E}+00$ & 580 \\
\hline U-233 & $4.83 E+04$ & $4.48 \mathrm{E}-11$ & $7.38 \mathrm{E}+02$ & $1.06 \mathrm{E}-02$ & 30,000 \\
\hline U-234 & $5.05 \mathrm{E}+04$ & $4.44 \mathrm{E}-11$ & $7.38 \mathrm{E}+02$ & $1.13 E-02$ & 30,000 \\
\hline U-235 & $5.50 \mathrm{E}+04$ & $4.70 \mathrm{E}-11$ & $7.38 \mathrm{E}+02$ & $1.38 \mathrm{E}-02$ & 34,000 \\
\hline U-236 & $5.49 \mathrm{E}+04$ & $4.21 \mathrm{E}-11$ & $7.38 \mathrm{E}+02$ & $1.24 \mathrm{E}-02$ & 31,000 \\
\hline $\mathrm{U}-238$ & $5.50 \mathrm{E}+04$ & $6.20 \mathrm{E}-11$ & $7.38 \mathrm{E}+02$ & $1.83 \mathrm{E}-02$ & 34,000 \\
\hline $\mathrm{Np}-237$ & $3.87 \mathrm{E}+04$ & $2.95 \mathrm{E}-10$ & $7.34 \mathrm{E}+02$ & $1.83 \mathrm{E}-02$ & 70,000 \\
\hline Pu-239 & $5.79 E+03$ & $3.16 \mathrm{E}-10$ & $7.33 \mathrm{E}+02$ & $1.12 \mathrm{E}-03$ & 64,000 \\
\hline $\mathrm{Pu}-240$ & $4.54 \mathrm{E}+01$ & $3.15 \mathrm{E}-10$ & $7.33 \mathrm{E}+02$ & $5.01 \mathrm{E}-07$ & 62,000 \\
\hline Am-241 & $2.80 \mathrm{E}-36$ & $3.28 \mathrm{E}-10$ & $7.34 \mathrm{E}+02$ & $4.74 \mathrm{E}-42$ & 55,000 \\
\hline
\end{tabular}

Note: The ratio of the dilution factors $\mathrm{DF}_{\text {well }}$ to the $\mathrm{DF}_{\text {creek }}$ is 0.17 . 
Table 2. Peak effective risks and doses for the EMWMF for hazardous constituents (based on a $1 \mathrm{~kg} / \mathrm{m}^{3}$ concentration in the waste)

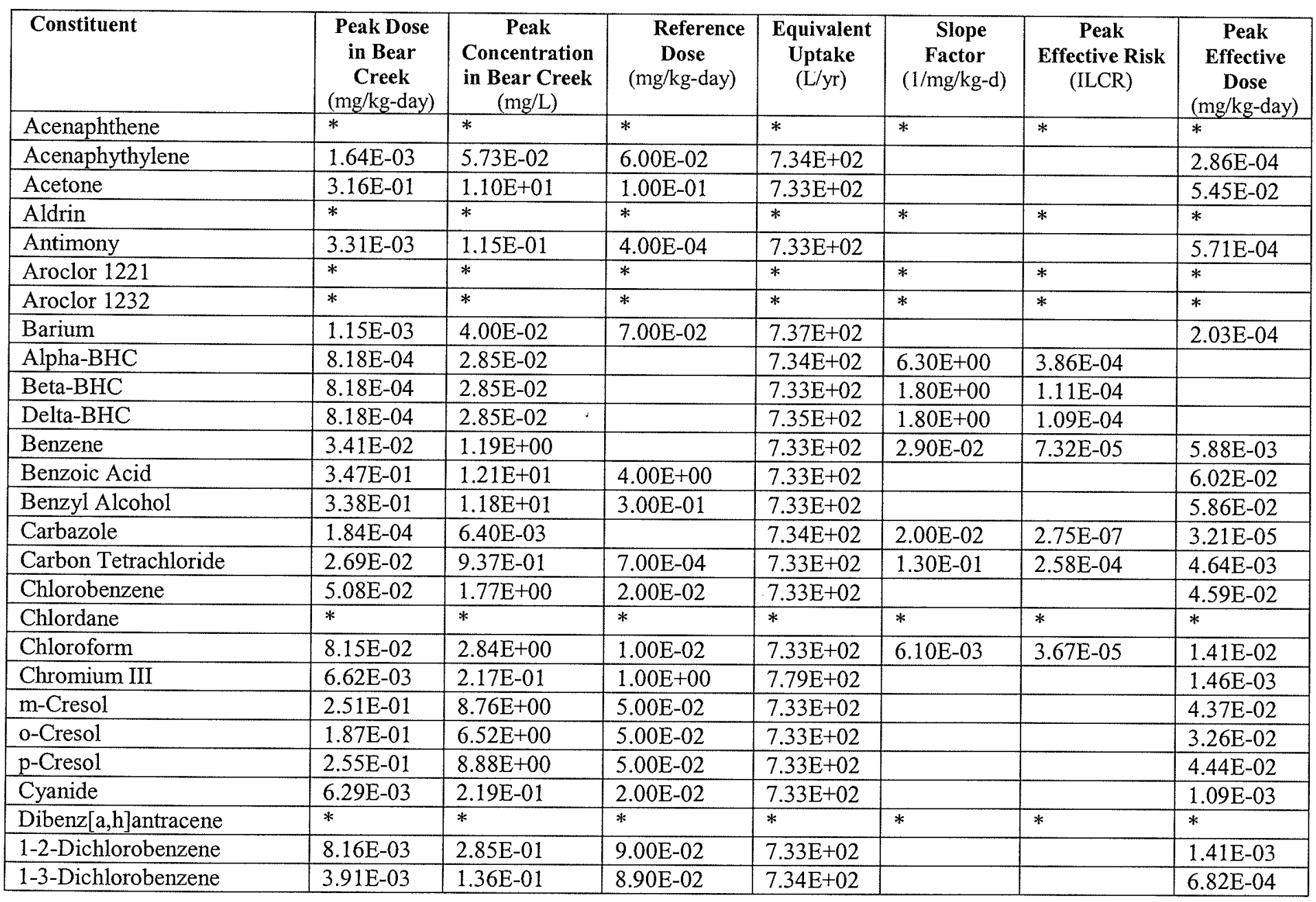


Table 2. Peak effective risks and doses for the EMWMF for hazardous constituents (based on a $1 \mathrm{~kg} / \mathrm{m}^{3}$ concentration in the waste)

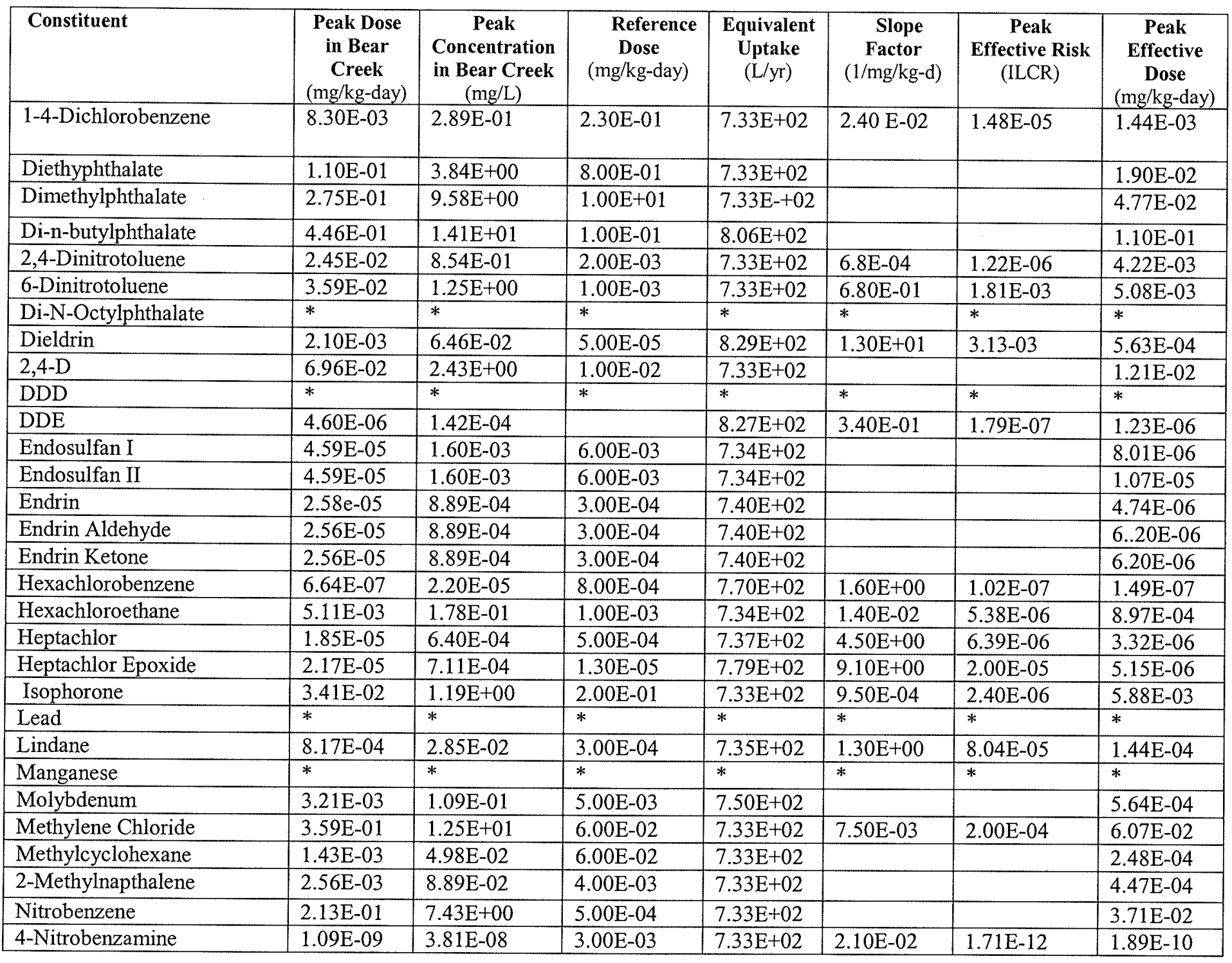


Table 2. Peak effective risks and doses for the EMWMF for hazardous constituents (based on a $1 \mathrm{~kg} / \mathrm{m}^{3}$ concentration in the waste)

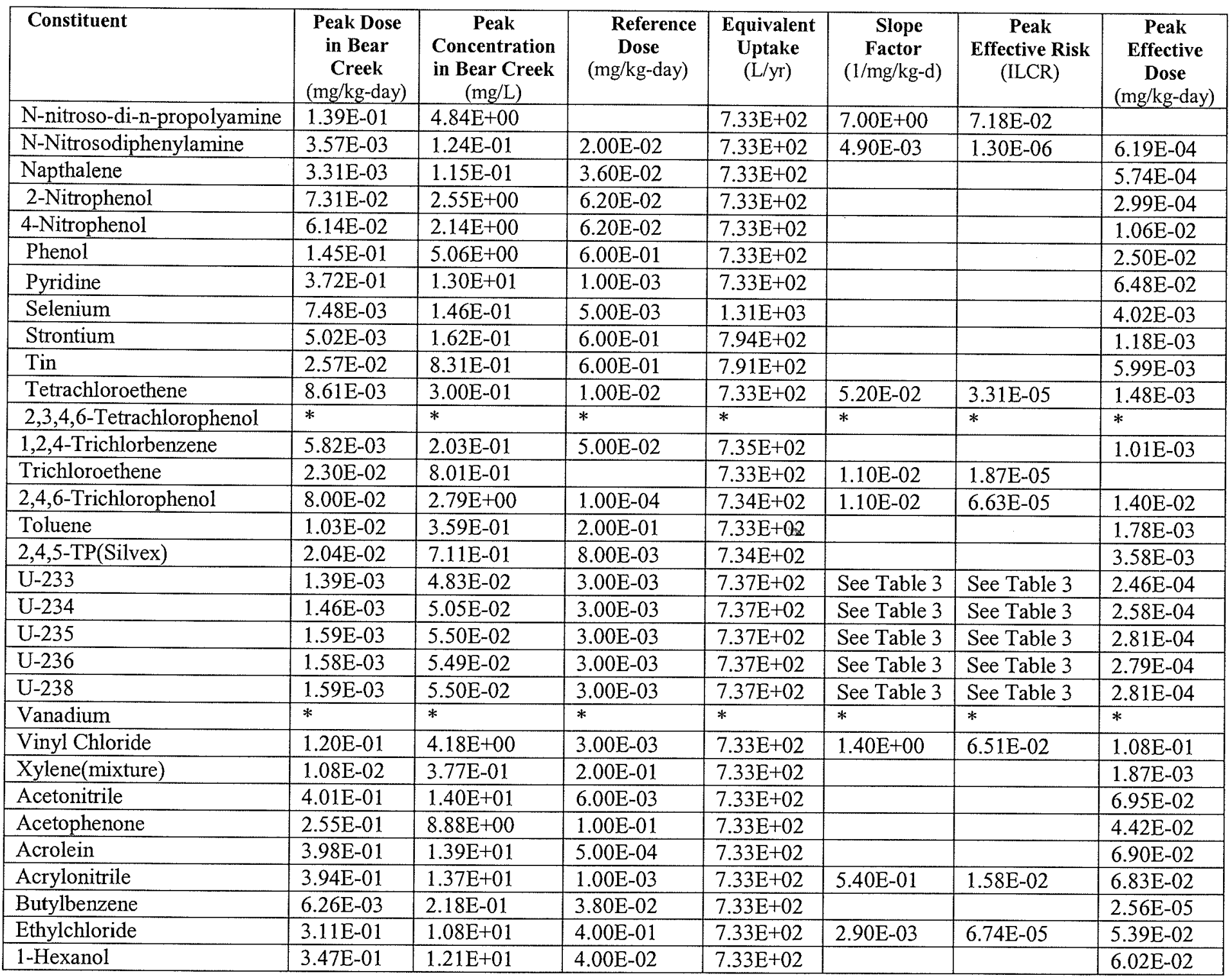


Table 2. Peak effective risks and doses for the EMWMF for hazardous constituents (based on a $1 \mathrm{~kg} / \mathrm{m}^{3}$ concentration in the waste)

\begin{tabular}{|c|c|c|c|c|c|c|c|}
\hline Constituent & $\begin{array}{c}\text { Peak Dose } \\
\text { in Bear } \\
\text { Creek } \\
\text { (mg/kg-day) } \\
\end{array}$ & $\begin{array}{c}\text { Peak } \\
\text { Concentration } \\
\text { in Bear Creek } \\
(\mathrm{mg} / \mathrm{L})\end{array}$ & $\begin{array}{c}\text { Reference } \\
\text { Dose } \\
\text { (mg/kg-day) }\end{array}$ & $\begin{array}{l}\text { Equivalent } \\
\text { Uptake } \\
\text { (L/yr) }\end{array}$ & $\begin{array}{c}\text { Slope } \\
\text { Factor } \\
(1 / \mathrm{mg} / \mathrm{kg}-\mathrm{d})\end{array}$ & $\begin{array}{c}\text { Peak } \\
\text { Effective Risk } \\
\text { (ILCR) }\end{array}$ & $\begin{array}{c}\text { Peak } \\
\text { Effective } \\
\text { Dose } \\
\text { (mg/kg-day) } \\
\end{array}$ \\
\hline 2-Hexanone & $3.47 \mathrm{E}-01$ & $1.21 \mathrm{E}+01$ & $4.00 \mathrm{E}-02$ & $7.33 \mathrm{E}+02$ & & & $6.02 \mathrm{E}-02$ \\
\hline (1-Methylpropl)benzene & $3.51 \mathrm{E}-05$ & $1.22 \mathrm{E}+01$ & $3.70 \mathrm{E}-02$ & $7.33 \mathrm{E}+02$ & & & $6.12 \mathrm{E}-06$ \\
\hline 2,4,6-Trichlorophenol & $2.37 \mathrm{E}-04$ & $8.00 \mathrm{E}-02$ & $0.00 \mathrm{E}+00$ & $7.4 \mathrm{E}+02$ & $1.10 \mathrm{E}-02$ & $6.63 \mathrm{E}-05$ & $1.40 \mathrm{E}-02$ \\
\hline $\begin{array}{l}\text { Trimethylbenzene } \\
\text { (mixed isomers) }\end{array}$ & $5.82 \mathrm{E}-03$ & $2.03 \mathrm{E}-01$ & $5.00 \mathrm{E}-02$ & $7.34 \mathrm{E}+02$ & & & $1.02 \mathrm{E}-03$ \\
\hline Dibenzofuran & $1.70 \mathrm{E}-01$ & $5.94 \mathrm{E}+00$ & $4.00 \mathrm{E}-03$ & $7.33 \mathrm{E}+02$ & & & $2.93 \mathrm{E}-02$ \\
\hline 2,4-Dimethylphenol & $2.37 \mathrm{E}-02$ & $8.25 \mathrm{E}-01$ & $2.00 \mathrm{E}-02$ & $7.33 \mathrm{E}+02$ & & & $4.77 \mathrm{E}-02$ \\
\hline Benzidine & $1.12 \mathrm{E}-02$ & $3.92 \mathrm{E}-01$ & $3.00 \mathrm{E}-03$ & $7.33 \mathrm{E}+02$ & $2.30 \mathrm{E}+02$ & $1.92 \mathrm{E}-01$ & $1.94 \mathrm{E}-03$ \\
\hline Methanol & $4.00 \mathrm{E}-01$ & $1.39 \mathrm{E}+01$ & $5.00 \mathrm{E}-01$ & $7.33 \mathrm{E}+02$ & & & $6.94 \mathrm{E}-02$ \\
\hline Methyl Metacrylate & $3.59 \mathrm{E}-01$ & $1.25 \mathrm{E}+01$ & $1.4 \mathrm{E}+00$ & $7.33 \mathrm{E}+02$ & & & $6.23 \mathrm{E}-02$ \\
\hline $\begin{array}{l}\text { Cumene } \\
\text { (Isopropylbenzene) }\end{array}$ & $3.51 \mathrm{E}-05$ & $1.22 \mathrm{E}-01$ & $1.00 \mathrm{E}-01$ & $7.33 \mathrm{E}+02$ & & & $6.12 \mathrm{E}-02$ \\
\hline $\begin{array}{l}\text { (1-Methyl- } \\
\text { propyl)benzene }\end{array}$ & $3.51 \mathrm{E}-05$ & $1.22 \mathrm{E}-01$ & $3.70 \mathrm{E}-02$ & $7.33 \mathrm{E}+02$ & & & $6.12 \mathrm{E}-02$ \\
\hline 1,2-Dimethyl-enzene & $9.95 \mathrm{E}-05$ & $3.47 \mathrm{E}-03$ & $2.00 \mathrm{E}+00$ & $7.33 \mathrm{E}+02$ & & & $1.74 \mathrm{E}-05$ \\
\hline $\begin{array}{l}\text { 1-Methyl-4-(1- } \\
\text { (methylethyl)benzene }\end{array}$ & $3.51 \mathrm{E}-05$ & $1.22 \mathrm{E}-03$ & $3.70 \mathrm{E}-02$ & $7.33 E+02$ & & & $6.12 \mathrm{E}-06$ \\
\hline Propylbenzene & $3.51 \mathrm{E}-05$ & $1.22 \mathrm{E}-03$ & $3.70 \mathrm{E}-02$ & $7.33 \mathrm{E}+02$ & & & $6.12 \mathrm{E}-06$ \\
\hline Bromodichloro-methane & $3.09 \mathrm{E}-01$ & $1.08 \mathrm{E}+01$ & $2.00 \mathrm{E}-02$ & $7.33 \mathrm{E}+02$ & $6.20 \mathrm{E}-02$ & $1.43 \mathrm{E}-03$ & $5.36 \mathrm{E}-02$ \\
\hline Bromoform & $1.55 \mathrm{E}-01$ & $5.41 \mathrm{E}-01$ & $2.00 \mathrm{E}-02$ & $7.33 \mathrm{E}+02$ & $7.90 \mathrm{E}-03$ & $9.11 \mathrm{E}-05$ & $2.69 \mathrm{E}-02$ \\
\hline Bromomethane & $3.43 \mathrm{E}-01$ & $1.20 \mathrm{E}+01$ & $1.40 \mathrm{E}-03$ & $7.33 \mathrm{E}+02$ & & & $5.95 \mathrm{E}-02$ \\
\hline Carbon Disulfide & $1.20 \mathrm{E}-01$ & $4.20 \mathrm{E}+00$ & $1.00 \mathrm{E}-01$ & $7.33 \mathrm{E}+02$ & & & $2.08 \mathrm{E}-02$ \\
\hline Chloromethane & $3.42 \mathrm{E}-01$ & $1.19 \mathrm{E}+01$ & & $7.33 \mathrm{E}+02$ & $1.30 \mathrm{E}-02$ & $3.30 \mathrm{E}-04$ & $5.93 \mathrm{E}-02$ \\
\hline o-Chlorotoluene & $3.82 \mathrm{E}-02$ & $1.33 \mathrm{E}+00$ & $2.00 \mathrm{E}-02$ & $7.33 \mathrm{E}+02$ & & & $6.64 \mathrm{E}-03$ \\
\hline Dibromochloromethane & $2.13 \mathrm{E}-01$ & $7.42 \mathrm{E}+00$ & $2.00 \mathrm{E}-02$ & $7.33 \mathrm{E}+02$ & $8.40 \mathrm{E}-02$ & $1.33 \mathrm{E}-03$ & $3.69 \mathrm{E}-02$ \\
\hline Dichlorodifluoromethane & $2.86 \mathrm{E}-02$ & $9.96 \mathrm{E}-01$ & $2.00 \mathrm{E}-01$ & $7.33 \mathrm{E}+02$ & & & $4.96 \mathrm{E}-03$ \\
\hline 1,2,-cis-Dichloroethylene & $5.49 \mathrm{E}-02$ & $1.92 \mathrm{E}+00$ & $1.00 \mathrm{E}-02$ & $7.33 \mathrm{E}+02$ & & & $9.52 \mathrm{E}-03$ \\
\hline 1,2-trans-Dichloroetylene & $2.73 \mathrm{E}-01$ & $9.50 \mathrm{E}+00$ & $2.00 \mathrm{E}-02$ & $7.33 \mathrm{E}+02$ & & & $4.73 \mathrm{E}-02$ \\
\hline 1,2-Dichloropropane & $2.53 \mathrm{E}-01$ & $8.82 \mathrm{E}+00$ & & $7.33 \mathrm{E}+02$ & $6.80 \mathrm{E}-02$ & $1.28 \mathrm{E}-03$ & 4.39E-02 \\
\hline
\end{tabular}


Table 2. Peak effective risks and doses for the EMWMF for hazardous constituents (based on a $1 \mathrm{~kg} / \mathrm{m}^{3}$ concentration in the waste)

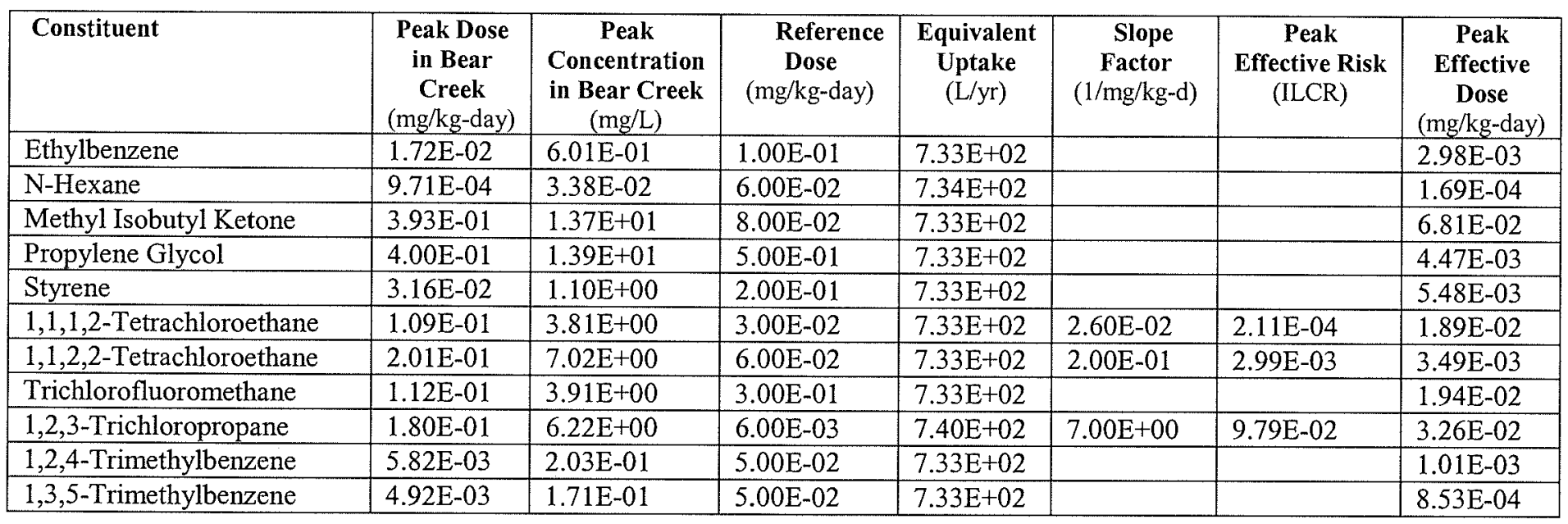

Note: The ratio of the well dilution factor to the creek dilution factor is 0.17 .

$*=$ Constituent not projected to appear within 100,000 years

$\mathrm{ILCR}=$ incremental lifetime cancer risk 
Table 3. Projected peak risks for EMWMF for radioactive constituents (risks based on contaminant concentrations in the waste equal to the current WAC)

\begin{tabular}{|c|c|c|c|}
\hline Nuclide & $\begin{array}{l}\text { Projected } \\
\text { Peak Risk } \\
\text { (ILCR) }\end{array}$ & $\begin{array}{l}\text { Risk } \\
\text { Criterion } \\
\text { (ILCR) }\end{array}$ & $\begin{array}{c}\text { Time of } \\
\text { Projected Peak Risk } \\
(\mathrm{yr})\end{array}$ \\
\hline $\mathrm{H}-3$ & $4.44 \mathrm{E}-09$ & $1.00 \mathrm{E}-05$ & 220 \\
\hline $\mathrm{C}-14$ & $4.86 \mathrm{E}-06$ & $1.00 \mathrm{E}-05$ & 270 \\
\hline Tc-99 & $2.34 \mathrm{E}-06$ & $1.00 \mathrm{E}-05$ & 300 \\
\hline I-129 & $4.60 \mathrm{E}-06^{*}$ & $1.00 \mathrm{E}-05$ & 580 \\
\hline $\mathrm{U}-233$ & $2.32 \mathrm{E}-05$ & $1.00 \mathrm{E}-04$ & 30,000 \\
\hline U-234 & $2.40 \mathrm{E}-05$ & $1.00 \mathrm{E}-04$ & 30,000 \\
\hline U-235 & $2.45 \mathrm{E}-05$ & $1.00 \mathrm{E}-04$ & 34,000 \\
\hline U-236 & $2.48 \mathrm{E}-05$ & $1.00 \mathrm{E}-04$ & 31,000 \\
\hline U-238 & $2.58 \mathrm{E}-05$ & $1.00 \mathrm{E}-04$ & 34,000 \\
\hline $\mathrm{Np}-237$ & $2.24 \mathrm{E}-05$ & $1.00 \mathrm{E}-04$ & 70,000 \\
\hline $\mathrm{Pu}-239$ & $7.99 \mathrm{E}-06$ & $1.00 \mathrm{E}-04$ & 64,000 \\
\hline $\mathrm{Pu}-240$ & $5.04 \mathrm{E}-07$ & $1.00 \mathrm{E}-04$ & 62,000 \\
\hline Am-241 & $1.12 \mathrm{E}-26$ & $1.00 \mathrm{E}-04$ & 55,000 \\
\hline
\end{tabular}

* = Based on an I-129 WAC of $2.9 \mathrm{pCi} / \mathrm{g}$

$\mathrm{ILCR}=$ incremental lifetime cancer risk 
Table 4. Projected peak risks and doses for EMWMF for hazardous constituents (risks and doses based on contaminant concentrations in the waste equal to the corresponding current WAC)

\begin{tabular}{|c|c|c|c|c|c|}
\hline Contaminant & $\begin{array}{c}\text { Time of } \\
\text { Projected } \\
\text { Peak (yr) }\end{array}$ & $\begin{array}{l}\text { Projected } \\
\text { Peak Risk } \\
\text { (ILCR) }\end{array}$ & $\begin{array}{l}\text { Risk Criterion } \\
\text { (ILCR) }\end{array}$ & $\begin{array}{c}\text { Projected } \\
\text { Peak Dose } \\
\text { (mg/kg-day) }\end{array}$ & $\begin{array}{c}\text { Dose } \\
\text { Criterion } \\
(\mathrm{mg} / \mathrm{kg} \text {-day) }\end{array}$ \\
\hline Acenaphthene & 180,000 & $*$ & $*$ & $*$ & $*$ \\
\hline Acenaphthylene & 26,000 & & & $4.2 \mathrm{E}-02$ & $1.8 \mathrm{E}-01$ \\
\hline Acetone & 450 & & & $2.4 \mathrm{E}-02$ & $1.0 \mathrm{E}-01$ \\
\hline Aldrin & 190,000 & $*$ & $*$ & * & $9.0 \mathrm{E}-05$ \\
\hline Antimony & 40.000 & & & $1.5 \mathrm{E}-04$ & $1.2 \mathrm{E}-03$ \\
\hline Aroclor 1221 & 530,000 & $*$ & $1.0 \mathrm{E}-04$ & $*$ & $*$ \\
\hline Aroclor 1232 & 100,000 & & $1.0 \mathrm{E}-04$ & & \\
\hline Barium & 100,000 & & & $4.9 \mathrm{E}-02$ & $7.0 \mathrm{E}-02$ \\
\hline Alpha $\mathrm{BHC}$ & 7,700 & $2.4 \mathrm{E}-05$ & $1.0 \mathrm{E}-04$ & & \\
\hline Beta-BHC & 9,300 & $2.5 \mathrm{E}-05$ & $1.0 \mathrm{E}-04$ & & \\
\hline Delta-BHC & 9,300 & $2.5 \mathrm{E}-05$ & $1.0 \mathrm{E}-04$ & & \\
\hline Benzene & 3,100 & $2.3 \mathrm{E}-05$ & $1.0 \mathrm{E}-04$ & & \\
\hline Benzoic Acid & 410 & & & $9.5 \mathrm{E}-01$ & $4.0+00$ \\
\hline Benzyl Alcohol & 180 & & & $1.1 \mathrm{E}-01$ & $3.0 \mathrm{E}-01$ \\
\hline Carbazole & 15,000 & $2.4 \mathrm{E}-05$ & $1.0 \mathrm{E}-04$ & & \\
\hline Carbon Tetrachloride & 3,900 & $2.3 \mathrm{E}-05$ & $1.0 \mathrm{E}-04$ & $4.9 \mathrm{E}-04$ & $2.1 \mathrm{E}-03$ \\
\hline Chlorobenzene & 1,200 & & & $2.4 \mathrm{E}-02$ & $6.0 \mathrm{E}-02$ \\
\hline Chlordane & 330,000 & * & $1.0 \mathrm{E}-04$ & $*$ & $1.5 \mathrm{E}-03$ \\
\hline Chloroform & 1,500 & $2.4 \mathrm{E}-06$ & $1.0 \mathrm{E}-04$ & $2.3 \mathrm{E}-03$ & $3.0 \mathrm{E}-02$ \\
\hline Chromium III & 21,000 & & & $3.3 \mathrm{E}-01$ & $3.0 \mathrm{E}+00$ \\
\hline m-Cresol & 670 & & & $1.2 \mathrm{E}-02$ & $5.0 \mathrm{E}-02$ \\
\hline 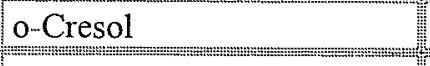 & 912 & & & $1.2 \mathrm{E}-02$ & $5.0 \mathrm{E}-02$ \\
\hline $\mathrm{p}$-Cresol & 660 & & & $1.2 \mathrm{E}-02$ & $5.0 \mathrm{E}-02$ \\
\hline Cyanide & 21,000 & & & $1.4 \mathrm{E}-02$ & $6.0 \mathrm{E}-02$ \\
\hline Dibenz $[\mathrm{a}, \mathrm{h}]$ anthracene & $>1,000,000$ & * & $*$ & $*$ & $*$ \\
\hline 1-2-Dichlorobenzene & 1,900 & & & $2.1 \mathrm{E}-02$ & $2.7 \mathrm{E}-01$ \\
\hline 1-3-Dichlorobenzene & 34,000 & & 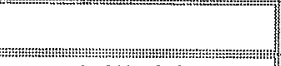 & $4.5 \mathrm{E}-02$ & $2.7 \mathrm{E}-01$ \\
\hline 1-4-Dichlorobenzene & 2,900 & $2.4 \mathrm{E}-06$ & $1.0 \mathrm{E}-04$ & $7.2 \mathrm{E}-02$ & $9.0 \mathrm{E}-01$ \\
\hline Diethylphthalate & 830 & & & $1.9 \mathrm{E}-01$ & $8.0 \mathrm{E}-01$ \\
\hline Dimethylphthalate & 610 & & & $2.3 \mathrm{E}+00$ & $1.0 \mathrm{E}+01$ \\
\hline Di-n-butyl phthalate & 400 & & & $3.3 \mathrm{E}-02$ & $1.0 \mathrm{E}-01$ \\
\hline 2,4-Dinitrotoluene & 2,200 & $2.0 \mathrm{E}-09$ & $1.0 \mathrm{E}-04$ & $4.2 \mathrm{E}-04$ & $6.0 \mathrm{E}-03$ \\
\hline 2,6-Dinitrotoluene & 640 & $2.4 \mathrm{E}-06$ & $1.0 \mathrm{E}-05$ & $2.0 \mathrm{E}-04$ & $1.0 \mathrm{E}-03$ \\
\hline Di-N-Octylphthalate & $>1,000,000$ & * & $*$ & $*$ & * \\
\hline Dieldrin & 57,000 & $3.6 \mathrm{E}-05$ & $1.0 \mathrm{E}-04$ & $5.3 \mathrm{E}-05$ & $1.5 \mathrm{E}-04$ \\
\hline $2,4 \mathrm{D}$ & 570 & & & $2.3 \mathrm{E}-03$ & $1.0 \mathrm{E}-02$ \\
\hline
\end{tabular}


Table 4. Projected peak risks and doses for EMWMF for hazardous constituents (risks and doses based on contaminant concentrations in the waste equal to the corresponding current WAC)

\begin{tabular}{|c|c|c|c|c|c|}
\hline Contaminant & $\begin{array}{l}\text { Time of } \\
\text { Projected } \\
\text { Peak (yr) }\end{array}$ & $\begin{array}{l}\text { Projected } \\
\text { Peak Risk } \\
\text { (ILCR) }\end{array}$ & $\begin{array}{l}\text { Risk Criterion } \\
\text { (ILCR) }\end{array}$ & $\begin{array}{l}\text { Projected } \\
\text { Peak Dose } \\
\text { (mg/kg-day) }\end{array}$ & $\begin{array}{c}\text { Dose } \\
\text { Criterion } \\
\text { (mg/kg-day) }\end{array}$ \\
\hline DDD & 180,000 & $*$ & $*$ & $*$ & $*$ \\
\hline DDE & 4,000 & $4.3 \mathrm{E}-05$ & $1.0 \mathrm{E}-04$ & & \\
\hline Endosulfan I & 8,900 & & & $4.2 \mathrm{E}-03$ & $1.8 \mathrm{E}-02$ \\
\hline Endosulfan II & 26,000 & & & $5.7 \mathrm{E}-03$ & $1.8 \mathrm{E}-02$ \\
\hline Endrin & 46,000 & & & $2.3 \mathrm{E}-04$ & $9.0 \mathrm{E}-04$ \\
\hline Endrin Aldehyde & 100,000 & & & $3.0 \mathrm{e}-04$ & $9.0 \mathrm{E}-04$ \\
\hline Endrin Ketone & 100,000 & & & $3.0 \mathrm{E}-04$ & $9.0 \mathrm{E}-04$ \\
\hline Hexachlorobenzene & 210,000 & $*$ & $*$ & * & $*$ \\
\hline 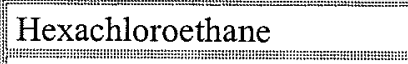 & 7,800 & $2.4 \mathrm{E}-05$ & $1.0 \mathrm{E}-04$ & $7.2 \mathrm{E}-04$ & $3.0 \mathrm{E}-03$ \\
\hline Heptachlor & 93,000 & $2.5 \mathrm{E}-05$ & $1.0 \mathrm{E}-04$ & $3.7 \mathrm{E}-04$ & $1.5 \mathrm{E}-03$ \\
\hline Heptachlor Epoxide & 37,000 & $3.2 \mathrm{E}-05$ & $1.0 \mathrm{E}-04$ & $1.5 \mathrm{E}-05$ & $3.9 \mathrm{E}-05$ \\
\hline Isophorone & 3,100 & $2.3 \mathrm{E}-05$ & $1.0 \mathrm{E}-04$ & $1.4 \mathrm{E}-01$ & $6.0 \mathrm{E}-01$ \\
\hline Lead & 190,000 & $*$ & $*$ & $*$ & $*$ \\
\hline Lindane & 15,000 & $2.3 \mathrm{E}-05$ & $1.0 \mathrm{E}-04$ & $2.2 \mathrm{E}-04$ & $9.0 \mathrm{E}-04$ \\
\hline Manganese & 390,000 & $*$ & $*$ & $*$ & $*$ \\
\hline Molybdenum & 42,000 & & & $3.58 \mathrm{E}-3$ & $1.5 \mathrm{E}-02$ \\
\hline Methylene Chloride & 460 & $2.3 \mathrm{E}-06$ & $1.0 \mathrm{E}-05$ & $1.4 \mathrm{E}-02$ & $6.0 \mathrm{E}-02$ \\
\hline Methylcyclohexane & 401 & & & $3.1 \mathrm{E}-03$ & $1.3 \mathrm{E}-02$ \\
\hline 2-Methyllapthalene & 13,000 & & & $2.3 \mathrm{E}-03$ & $1.2 \mathrm{E}-02$ \\
\hline Nitrobenzene & 576 & & & $1.2 \mathrm{E}-04$ & $5.0 \mathrm{E}-04$ \\
\hline 4-Nitrobenzamine & 1,000 & $2.4 \mathrm{E}-05$ & $1.0 \mathrm{E}-04$ & $2.1 \mathrm{E}-04$ & $9.0 \mathrm{E}-03$ \\
\hline $\begin{array}{l}\text { N-nitroso-di-n- } \\
\text { propolyamine }\end{array}$ & 930 & $2.2 \mathrm{E}-06$ & $1.0 \mathrm{E}-05$ & & \\
\hline N-Nitrosodiphenylamine & 1,700 & $2.2 \mathrm{E}-06$ & $1.0 \mathrm{E}-04$ & $4.8 \mathrm{E}-03$ & $6.0 \mathrm{E}-02$ \\
\hline Napthalene & 8,000 & & & $9.2 \mathrm{E}-03$ & $1.1 \mathrm{E}-01$ \\
\hline 2-Nitrophenol & 1,800 & & & $5.5 \mathrm{E}-07$ & $1.2 \mathrm{E}-01$ \\
\hline 4-Nitrophenol & 2,100 & & & $9.4 \mathrm{E}-03$ & $1.2 \mathrm{E}-01$ \\
\hline Phenol & 890 & & & $1.3 \mathrm{E}-01$ & $6.0 \mathrm{E}-01$ \\
\hline Pyridine & 440 & & & $2.3 \mathrm{E}-04$ & $1.0 \mathrm{E}-03$ \\
\hline Selenium & 32,000 & & & $1.0 \mathrm{E}-02$ & $1.5 \mathrm{E}-02$ \\
\hline Strontium & 23,000 & & & $1.5 \mathrm{E}-01$ & $1.8 \mathrm{E}+00$ \\
\hline $\operatorname{Tin}$ & 5,600 & & & $2.1 \mathrm{E}-02$ & $1.8 \mathrm{E}+00$ \\
\hline Tetrachloroethene & 12,000 & $2.3 \mathrm{E}-05$ & $1.0 \mathrm{E}-04$ & $6.9 \mathrm{E}-03$ & $3.0 \mathrm{E}-02$ \\
\hline $2,3,4,6$-Tetrachlorophenol & 480,000 & $*$ & $*$ & $*$ & $*$ \\
\hline 1,2,4-Trichlorbenzene & 3,300 & & & $8.2 \mathrm{E}-03$ & $3.0 \mathrm{E}-02$ \\
\hline Trichloroethene & 4,600 & $2.3 \mathrm{E}-05$ & $1.0 \mathrm{E}-04$ & & \\
\hline 2,4,6-Trichlorophenol & 1,600 & $2.3 \mathrm{E}-05$ & $1.0 \mathrm{E}-04$ & a & $3.0 \mathrm{E}-04$ \\
\hline
\end{tabular}


Table 4. Projected peak risks and doses for EMWMF for hazardous constituents (risks and doses based on contaminant concentrations in the waste equal to the corresponding current WAC)

\begin{tabular}{|c|c|c|c|c|c|}
\hline Contaminant & $\begin{array}{l}\text { Time of } \\
\text { Projected } \\
\text { Peak (yr) }\end{array}$ & $\begin{array}{l}\text { Projected } \\
\text { Peak Risk } \\
\text { (ILCR) }\end{array}$ & $\begin{array}{l}\text { Risk Criterion } \\
\text { (ILCR) }\end{array}$ & $\begin{array}{c}\text { Projected } \\
\text { Peak Dose } \\
\text { (mg/kg-day) }\end{array}$ & $\begin{array}{c}\text { Dose } \\
\text { Criterion } \\
\text { (mg/kg-day) }\end{array}$ \\
\hline Toluene & 10,000 & & & $1.4 \mathrm{E}-01$ & $6.0 \mathrm{E}-01$ \\
\hline 2,4,5-TP (Silvex) & 850 & & & $4.5 \mathrm{E}-04$ & $8.0 \mathrm{E}-03$ \\
\hline U-233 & 30,000 & See Table 2 & & $6.2 \mathrm{E}-04$ & $3.0 \mathrm{E}-03$ \\
\hline U-234 & 30,000 & See Table 2 & & $62 \mathrm{E}-04$ & $3.0 \mathrm{E}-03$ \\
\hline U-235 & 34,000 & See Table 2 & & $6.6 \mathrm{E}-04$ & $3.0 \mathrm{E}-03$ \\
\hline U-236 & 31,000 & See Table 2 & & $6.6 \mathrm{E}-04$ & $3.0 \mathrm{E}-03$ \\
\hline U-238 & 34,000 & See Table 2 & & $6.8 \mathrm{E}-04$ & $3.0 \mathrm{E}-03$ \\
\hline Vanadium & 190,000 & $*$ & $*$ & * & $*$ \\
\hline Vinyl Chloride & 1,100 & $3.0 \mathrm{E}-06$ & $1.0 \mathrm{E}-05$ & $2.6 \mathrm{E}-04$ & $3.0 \mathrm{E}-03$ \\
\hline Xylene (mixture) & 2,200 & & & $4.5 \mathrm{E}-02$ & $6.0 \mathrm{E}-01$ \\
\hline Acetonitrile & 410 & & & $1.4 \mathrm{E}-03$ & $6.0 \mathrm{E}-03$ \\
\hline Acetophenone & 660 & & & $2.3 \mathrm{E}-02$ & $1.0 \mathrm{E}-01$ \\
\hline Acrolein & 410 & & & $1.2 \mathrm{E}-04$ & $5.0 \mathrm{E}-04$ \\
\hline Acrylonitrile & 410 & $2.4 \mathrm{E}-06$ & $1.0 \mathrm{E}-05$ & $2.3 \mathrm{E}-04$ & $1.0 \mathrm{E}-03$ \\
\hline Butylbenzene & 3,700 & & & $6.0 \mathrm{E}-04$ & $1.1 \mathrm{E}-01$ \\
\hline Ethylchloride & 540 & $1.9 \mathrm{E}-06$ & $1.0 \mathrm{E}-05$ & $9.5 \mathrm{E}-02$ & $4.0 \mathrm{E}-01$ \\
\hline 1-Hexanol & 475 & & & $9.3 \mathrm{E}-03$ & $4.0 \mathrm{E}-02$ \\
\hline 2-Hexanone & 475 & & & $9.3 \mathrm{E}-03$ & $4.0 \mathrm{E}-02$ \\
\hline $\begin{array}{l}\text { Trimethylbenzene } \\
\text { (mixed isomers) }\end{array}$ & 3,300 & & & $3.6 \mathrm{E}-02$ & $1.5 \mathrm{E}-01$ \\
\hline Dibenzofuran & 440,000 & $*$ & $*$ & $*$ & $*$ \\
\hline 2,4-Dimethylphenol & 5,600 & & & $1.9 \mathrm{E}-02$ & $6.0 \mathrm{E}-02$ \\
\hline Benzidine & 12,000 & $5.0 \mathrm{E}-05$ & $1.0 \mathrm{E}-04$ & $3.7 \mathrm{E}-05$ & $6.0 \mathrm{E}-02$ \\
\hline Methanol & 410 & & & $1.2 \mathrm{E}-1$ & $5.0 \mathrm{E}-01$ \\
\hline Methyl Metacrylate & 170 & & & $3.3 \mathrm{E}-01$ & $1.4 \mathrm{E}+00$ \\
\hline $\begin{array}{l}\text { Cumene } \\
\text { (Isopropyl-benzene) }\end{array}$ & 3,800 & & & $4.0 \mathrm{E}-04$ & $3.00 \mathrm{E}-1$ \\
\hline $\begin{array}{l}\text { (1-Methyl- } \\
\text { propyl)-benzene }\end{array}$ & 3,800 & & & $1.5 \mathrm{E}-05$ & $1.1 \mathrm{E}-02$ \\
\hline 1,2-Dimethyl-benzene & 1,300 & & & $2.1 \mathrm{E}-03$ & $6.0 \mathrm{E}-02$ \\
\hline (1-Methyl-4- & 3,800 & & & $1.5 \mathrm{E}-04$ & $1.1 \mathrm{E}-01$ \\
\hline Propylbenzene & 3,800 & & & $1.5 \mathrm{E}-04$ & $1.1 \mathrm{E}-01$ \\
\hline Bromodichloro-methane & 400 & $2.3 \mathrm{E}-06$ & $1.0 \mathrm{E}-05$ & $4.7 \mathrm{E}-03$ & $2.0 \mathrm{E}-02$ \\
\hline Bromoform & 830 & $2.4 \mathrm{E}-06$ & $1.0 \mathrm{E}-05$ & $1.5 \mathrm{E}-02$ & $2.0 \mathrm{E}-02$ \\
\hline Bromomethane & 480 & & & $3.3 \mathrm{E}-04$ & $1.4 \mathrm{E}-03$ \\
\hline Carbon Disulfide & 700 & & & $2.3 \mathrm{E}-02$ & $1.00 \mathrm{E}-01$ \\
\hline Chloromethane & 480 & & & $2.5 \mathrm{E}-02$ & $1.0 \mathrm{E}-01$ \\
\hline
\end{tabular}


Table 4. Projected peak risks and doses for EMWMF for hazardous constituents (risks and doses based on contaminant concentrations in the waste equal to the corresponding current WAC)

\begin{tabular}{|c|c|c|c|c|c|}
\hline Contaminant & $\begin{array}{l}\text { Time of } \\
\text { Projected } \\
\text { Peak (yr) }\end{array}$ & $\begin{array}{l}\text { Projected } \\
\text { Peak Risk } \\
\text { (ILCR) }\end{array}$ & $\begin{array}{l}\text { Risk Criterion } \\
\text { (ILCR) }\end{array}$ & $\begin{array}{c}\text { Projected } \\
\text { Peak Dose } \\
\text { (mg/kg-day) }\end{array}$ & $\begin{array}{c}\text { Dose } \\
\text { Criterion } \\
\text { (mg/kg-day) }\end{array}$ \\
\hline o-Chlorotoluene & 2,200 & & & 4.7E-03 & $2.0 \mathrm{E}-02$ \\
\hline Dibromochloro-methane & 800 & $2.3 \mathrm{E}-06$ & $1.0 \mathrm{E}-05$ & $4.7 \mathrm{E}-03$ & $2.0 \mathrm{E}-02$ \\
\hline Dichlorodifluoro-methane & 440 & & & $4.8 \mathrm{E}-02$ & $2.0 \mathrm{E}-01$ \\
\hline 1,2,-cis-Dichloroethylene & 2,300 & & & $2.3 \mathrm{E}-02$ & $1.0 \mathrm{E}-01$ \\
\hline 1,2-trans-Dichloroetylene & 620 & & & $4.7 \mathrm{E}-03$ & $2.0 \mathrm{E}-02$ \\
\hline 1,2-Dichloropropane & 580 & $2.2 \mathrm{E}-06$ & $1.0 \mathrm{E}-05$ & & \\
\hline Ethylbenzene & 1,200 & & & $7.0 \mathrm{E}-02$ & $3.0 \mathrm{E}-01$ \\
\hline N-Hexane & 930 & & & $1.4 \mathrm{E}-02$ & $6.0 \mathrm{E}-03$ \\
\hline Methyl Isobutyl Ketone & 420 & & & $1.9 \mathrm{E}-01$ & $8.0 \mathrm{E}-01$ \\
\hline Propylene Glycol & 410 & & & $7.9 \mathrm{E}-3$ & $1.25 \mathrm{E}+01$ \\
\hline Styrene & 4,100 & & & $4.2 \mathrm{E}-01$ & $6.0 \mathrm{E}-01$ \\
\hline 1,1,1,2-Tetrachloroethane & 970 & $243 \mathrm{E}-06$ & $1.0 \mathrm{E}-05$ & $6.9 \mathrm{E}-03$ & $3.0 \mathrm{E}-02$ \\
\hline 1,1,2,2-Tetrachloroethane & 730 & $1.7 \mathrm{E}-07$ & $1.0 \mathrm{E}-05$ & $1.2 \mathrm{E}-05$ & $6.0 \mathrm{E}-03$ \\
\hline Trichlorofluoro-methane & 1,100 & & & $2.1 \mathrm{E}-01$ & $9.0 \mathrm{E}-01$ \\
\hline 1,2,3-Trichloropropane & 850 & $2.5 \mathrm{E}-06$ & $1.0 \mathrm{E}-05$ & $1.5 \mathrm{E}-03$ & $6.0 \mathrm{E}-03$ \\
\hline $\begin{array}{l}1,2,4- \\
\text { Trimethylbenzene }\end{array}$ & 3,800 & & & $3.5 \mathrm{E}-02$ & $1.5 \mathrm{E}-01$ \\
\hline 1,3,5-Trimethylbenzene &, 7300 & & & $3.5 \mathrm{E}-02$ & $1.5 \mathrm{E}-01$ \\
\hline
\end{tabular}

$\mathrm{ILCR}=$ incremental lifetime cancer risk

* = Constituent not projected to appear within 100,000 years.

$a=$ Both a slope factor and a reference dose exist for this constituent but there is no dose-based WAC for it given in Revised Table A.1: Analytic WAC Limits, http://bechteljacobs.org/webindex.html\#3.. 


\section{REFERENCES}

BJC 2003. Engineering Feasibility Plan for Groundwater Suppression at the Environmental Management Waste Management Facility, Oak Ridge, Tennessee, BJC10R-1478-R1, Oak Ridge, TN.

DOE 1998a. Remedial Investigation/Feasibility Study for the Disposal of Oak Ridge Reservation Comprehensive Environmental Response, Compensation, and Liability Act of 1980 Waste, DOE/OR02-1637 \& D2. Oak Ridge, TN.

DOE 1998b. Addendum to Remedial Investigation/Feasibility Study for the Disposal of Oak Ridge Reservation Comprehensive Environmental Response, Compensation, and Liability Act of 1980 Waste, DOE/OR/02-1637 \& D2/AL. Oak Ridge, TN.

DOE 2001a. Attainment Plan for Risk/Toxicity-Based Waste Acceptance Criteria at the Oak Ridge Reservation, Oak Ridge, Tennessee, DOE/OR/01-1909\&D3. Bechtel Jacobs Company LLC, Oak Ridge, TN.

DOE 2001b. Remedial Design Report for the Disposal of Oak Ridge Reservation Comprehensive Environmental Response, Compensation, and Liability Act of 1980 Waste, Oak Ridge, Tennessee, DOE/OR/01-1987\&D2, Oak Ridge, TN.

DOE 2003. Addendum to Remedial Design Report for the Disposal of Oak Ridge Reservation Comprehensive Environmental Response, Compensation, and Liability Act of 1980 Waste, DOE/OR/02-1873\&D2/A2/R1. Oak Ridge, TN.

RAE 1995a. The PATHRAE-RAD Performance Assessment Code for the Land Disposal of Radioactive Wastes, Rogers and Associates Engineering Corporation, RAE-9500/2-1, Salt Lake City, UT.

RAE 1995b. The PATHRAE-HAZ Performance Assessment Code for the Land Disposal of Hazardous Chemical Wastes, Rogers and Associates Engineering Corporation, RAE9500/2-2, Salt Lake City, UT. 
ATTACHMENT 1

PATHRAE ENVIRONMENTAL TRANSPORT ANALYSIS INPUT AND OUTPUT FOR THE EMWMF 


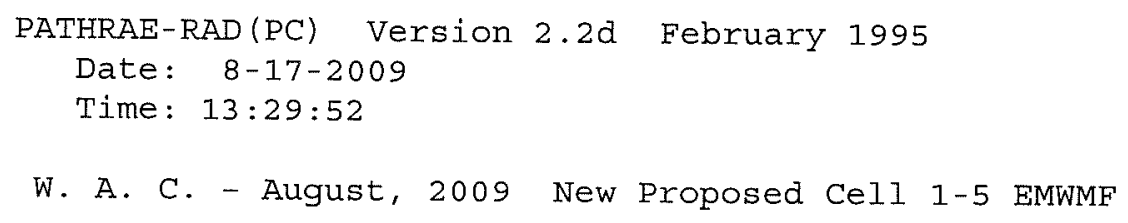




$\begin{array}{llll}\text { 111, Ba-133 } & 3.4 \mathrm{E}-06, & 6.9 \mathrm{E}-06, & 4.2 \mathrm{E}-05, \\ 112, \mathrm{Eu}-152 & 6.5 \mathrm{E}-06, & 2.2 \mathrm{E}-04, & 1.28 \mathrm{E}-04, \\ 113, \mathrm{Eu}-154 & 9.5 \mathrm{E}-06, & 2.9 \mathrm{E}-04, & 1.39 \mathrm{E}-04, \\ 114, \mathrm{Eu}-155 & 1.5 \mathrm{E}-06, & 4.1 \mathrm{E}-05, & 6.89 \mathrm{E}-06, \\ 055, \mathrm{Ra}-226 & 8.6 \mathrm{E}-03, & 7.9 \mathrm{E}-03, & 7.6 \mathrm{E}-07, \\ 116, \mathrm{Th}-228 & 3.8 \mathrm{E}-04, & 3.1 \mathrm{E}-01, & 2.8 \mathrm{E}-07, \\ 051, \mathrm{Th}-229 & 4.0 \mathrm{E}-03, & 2.0 \mathrm{E}+00, & 1.0 \mathrm{E}-05, \\ 036, \mathrm{Th}-230 & 5.5 \mathrm{E}-04, & 3.2 \mathrm{E}-01, & 9.1 \mathrm{E}-08, \\ 037, \mathrm{Th}-232 & 5.0 \mathrm{E}-03, & 1.6 \mathrm{E}+00, & 6.44 \mathrm{E}-08, \\ 053, \mathrm{~Pa}-231 & 2.5 \mathrm{E}-02, & 1.3 \mathrm{E}+00, & 3.6 \mathrm{E}-06, \\ 121, \mathrm{U}-232 & 1.31 \mathrm{E}-03, & 1.4 \mathrm{E}-01, & 8.36 \mathrm{E}-08, \\ 054, \mathrm{U}-233 & 2.89 \mathrm{E}-04, & 1.4 \mathrm{E}-01, & 8.36 \mathrm{E}-08, \\ 038, \mathrm{U}-234 & 2.83 \mathrm{E}-04, & 1.3 \mathrm{E}-01, & 8.74 \mathrm{E}-08, \\ 039, \mathrm{U}-235 & 2.67 \mathrm{E}-04, & 1.2 \mathrm{E}-01, & 1.73 \mathrm{E}-05, \\ 040, \mathrm{U}-236 & 2.69 \mathrm{E}-04, & 1.3 \mathrm{E}-01, & 7.59 \mathrm{E}-08, \\ 041, \mathrm{U}-238 & 2.7 \mathrm{E}-04, & 1.2 \mathrm{E}-01, & 2.82 \mathrm{E}-06, \\ 042, \mathrm{~Np}-237 & 4.4 \mathrm{E}-03, & 4.9 \mathrm{E}-01, & 3.2 \mathrm{E}-06, \\ 043, \mathrm{Pu}-238 & 3.2 \mathrm{E}-03, & 3.9 \mathrm{E}-01, & 9.79 \mathrm{E}-08, \\ 044, \mathrm{Pu}-239 & 3.5 \mathrm{E}-03, & 4.3 \mathrm{E}-01, & 4.29 \mathrm{E}-08, \\ 045, \mathrm{Pu}-240 & 3.5 \mathrm{E}-03, & 5.1 \mathrm{E}-01, & 8.2 \mathrm{E}-08, \\ 048, \mathrm{Am}-241 & 3.6 \mathrm{E}-03, & 4.4 \mathrm{E}-01, & 3.21 \mathrm{E}-06, \\ 132, \mathrm{Cm}-243 & 2.5 \mathrm{E}-03, & 3.1 \mathrm{E}-01, & 1.46 \mathrm{E}-05, \\ 050, \mathrm{Cm}-244 & 2.0 \mathrm{E}-03, & 2.5 \mathrm{E}-01, & 1.03 \mathrm{E}-07, \\ 020, \mathrm{I}-129 & 2.8 \mathrm{E}-04, & 1.8 \mathrm{E}-04, & 2.2 \mathrm{E}-06, \\ 025, \mathrm{Be}-10 & 4.2 \mathrm{E}-06, & 3.5 \mathrm{E}-04, & 0.0 \mathrm{E}-00\end{array}$

- Input File: INVNTRY.DAT

$101,1.23 \mathrm{E}+01,1.324 \mathrm{E}+06, .0, .000$,

$102,5.73 \mathrm{E}+03,1.324 \mathrm{E}+06, .0, .000$,

$103,1.28 \mathrm{E}+09,1.324 \mathrm{E}+06,10.3, .986$,

$104,7.42 \mathrm{E}-01,1.324 \mathrm{E}+06,24.7, .125$,

$105,5.27 \mathrm{E}+00,1.324 \mathrm{E}+06,9.2,1.253$,

$106,2.86 \mathrm{E}+01,1.324 \mathrm{E}+06, .0, .000$,

$107,9.60 \mathrm{E}-02,1.324 \mathrm{E}+06,11.6, .765$,

$108,2.13 \mathrm{E}+05,1.324 \mathrm{E}+06,29.2, .089$,

$109,2.06 \mathrm{E}+00,1.324 \mathrm{E}+06,12.1, .700$,

$110,3.02 \mathrm{E}+01,1.324 \mathrm{E}+06,12.8, .615$,

$111,1.07 \mathrm{E}+01,1.324 \mathrm{E}+06,22.2, .154$,

$\begin{array}{llll}0 ., & 0 ., & 1 ., & \mathrm{H}-3 \\ 0 ., & 0 ., & 1 ., & \mathrm{C}-14 \\ 0 ., & 0 ., & 1 ., & \mathrm{K}-40 \\ 0 ., & 0 ., & 1 ., & \mathrm{C}-57 \\ 0 ., & 0 ., & 1 ., & \mathrm{CO}-60 \\ 0 ., & 0 ., & 1 ., & \mathrm{Sr}-90 \\ 0 ., & 0 ., & 1 ., & \mathrm{Nb}-95 \\ 0 ., & 0 ., & 1 ., & \mathrm{TC}-99 \\ 0 ., & 0 ., & 1 ., & \mathrm{CS}-134 \\ 0 ., & 0 ., & 1 ., & \mathrm{CS}-137 \\ 0 ., & 0 ., & 1 ., & \mathrm{Ba}-133\end{array}$




\begin{tabular}{|c|c|c|c|c|c|c|c|c|}
\hline 12, & $36 E+0$ & 1 & 14.0 & .497 & 0. & 0. & 1. & Eu-152 \\
\hline 113, & $8.80 \mathrm{E}+00$, & 1. $324 \mathrm{E}+06$, & 12.5 & .657 & 0. & 0. & 1. & $E u-154$ \\
\hline 114, & 4. $96 \mathrm{E}+00$, & $1.324 \mathrm{E}+06$ & 32.1 & .078, & 0. & 0. & 1. & $E u-155$ \\
\hline 55, & 1. $60 \mathrm{E}+03$, & 1. $324 E+06$, & 21.5 & .170 & 0. & 0. & 1. & $R a-226$ \\
\hline 16, & 1. $91 \mathrm{E}+00$, & 1. $324 E+06$, & 25.9 & .112, & 0. & 0. & 1. & $T h-228$ \\
\hline 51 & $7.34 \mathrm{E}+03$, & $1.324 \mathrm{E}+06$ & 28.8 & .091 & 0. & 0. & 1. & Th-229 \\
\hline 036 & $7.70 \mathrm{E}+04$, & 1. $324 \mathrm{E}+06$, & 30.3 & .084 & 0. & 0. & 1. & Th-230 \\
\hline 37, & 1. $40 \mathrm{E}+10$, & 1.324E+06, & 35.5 , & .070 & 0. & 0. & 1. & Th-232 \\
\hline 053, & 3. $28 E+04$ & $1.324 E+06$, & 22.8 & .146 & 0. & 0. & 1. & $\mathrm{~Pa}-231$ \\
\hline 121, & 7. $20 \mathrm{E}+01$, & $1.324 \mathrm{E}+06$, & 25.7, & .000 & 0. & 0. & 1. & $\mathrm{U}-232$ \\
\hline 54, & 1.59E+05, & 1. $324 \mathrm{E}+06$, & 25.7 & .115 & 0. & 0. & 1. & $U-233$ \\
\hline 038, & $2.44 E+05$ & $1.324 \mathrm{E}+06$, & 35.5 & .070 & 0. & 0. & 1. & $\mathrm{U}-234$ \\
\hline 39 & $7.04 \mathrm{E}+08$ & $1.324 \mathrm{E}+06$, & 21.6, & .169 & 0. & 0. & 1. & $\mathrm{U}-235$ \\
\hline 40, & $2.34 \mathrm{E}+07$, & 1. $324 \mathrm{E}+06$, & 36.6 & .068 & 0. & 0. & $1 .$, & $U-236$ \\
\hline 41, & $4.47 \mathrm{E}+09$ & 1. $324 \mathrm{E}+06$, & 12.0 & .718, & 0. & 0. & $1 .$, & $\mathrm{U}-238$ \\
\hline 42 & $2.14 \mathrm{E}+06$, & 1. $324 \mathrm{E}+06$, & 34.9 & .072 & 0. & 0. & 1. & $\mathrm{~Np}-237$ \\
\hline 043 & $8.78 \mathrm{E}+01$, & 1. $324 \mathrm{E}+06$, & 45.3 & .055 & 0. & 0. & 1. & $\mathrm{Pu}-238$ \\
\hline 044 & $2.41 E+04$, & 1. $324 \mathrm{E}+06$, & 25.8, & .113 & $0 .$, & 0. & 1. & $P u-239$ \\
\hline 045, & $6.54 \mathrm{E}+03$ & 1. $324 \mathrm{E}+06$, & 46.3 & .054 & 0. & 0. & 1. & $P u-240$ \\
\hline 048, & $4.32 \mathrm{E}+02$ & 1. $324 \mathrm{E}+06$, & 43.5 & .057 & 0. & 0. & 1. & Am-241 \\
\hline 132 & $2.85 E+01$, & 1. $324 \mathrm{E}+06$, & 22.0 & .159, & 0. & $0 .$, & 1. & $\mathrm{Cm}-243$ \\
\hline 050, & 1. $81 \mathrm{E}+01$, & 1. $324 E+06$, & 43.5 & .057 & $0 .$, & 0. & $1 .$, & $\mathrm{Cm}-244$ \\
\hline 020 & $1.60 \mathrm{E}+07$, & 1.324E+06, & 62.0, & .040 & $.0 e-02$ & 0. & 1. & $I-129$ \\
\hline 25 & 1. $60 \mathrm{E}+07$, & $1.324 E+06$ & $.0 ，$ & .000, & $0 .$, & 0. & 1. & $\mathrm{Be}-10$ \\
\hline
\end{tabular}

- Input File: RQSITE.DAT

$101,-1.99 \mathrm{e}-1,0.00 \mathrm{E}+0,0.00 \mathrm{E}+0, \mathrm{H}-3$

$102,-1.09 e+0,0.00 \mathrm{E}+0,0.00 \mathrm{E}+0, \mathrm{C}-14$

$103,-3.98 \mathrm{e}+0,3.00 \mathrm{E}+0,3.00 \mathrm{E}+1, \mathrm{~K}-40$

$104,-3.97 \mathrm{e}+6,8.00 \mathrm{E}+1,8.00 \mathrm{E}+2, \mathrm{CO}-57$

$105,-3.97 e+6,8.00 \mathrm{E}+1,8.00 \mathrm{E}+2$, Co-60

$106,-8.74 e+0,3.00 E+0,3.00 E+1, \quad S r-90$

$107,-2.38 \mathrm{e}+1,3.79 \mathrm{E}+1,3.79 \mathrm{E}+2, \mathrm{Nb}-95$

$108,-1.29 \mathrm{e}+0,0.00 \mathrm{E}+0,0.00 \mathrm{E}+0, \mathrm{TC}-99$

$109,-1.99 \mathrm{e}+1,9.69 \mathrm{E}+1,9.69 \mathrm{E}+2, \mathrm{Cs}-134$

$110,-1.99 \mathrm{e}+1,3.00 \mathrm{E}+2,3.00 \mathrm{E}+3, \mathrm{Cs}-137$

111, $-55 ., 5.50 \mathrm{E}+0,5.50 \mathrm{E}+1, \mathrm{Ba}-133$

$112,-3.78 e+0,4.00 E+0,4.00 E+1, \quad \mathrm{Eu}-152$ 
$113,-3.78 e+0,4.00 E+0,4.00 E+1, \quad E u-154$

$114,-3.78 e+0,4.00 E+0,4.00 E+1, \quad E u-155$

$055,-1.99 e+1,3.00 E+2,3.00 E+3, \quad R a-226$

$116,-5.36 \mathrm{e}+1,3.00 \mathrm{E}+2,3.00 \mathrm{E}+3, \mathrm{Th}-228$

$051,-5.36 \mathrm{e}+1,3.00 \mathrm{E}+2,3.00 \mathrm{E}+3, \mathrm{Th}-229$

$036,-5.36 \mathrm{e}+1,3.00 \mathrm{E}+2,3.00 \mathrm{E}+3, \mathrm{Th}-230$

$037,-5.36 \mathrm{e}+1,3.00 \mathrm{E}+2,3.00 \mathrm{E}+3, \mathrm{Th}-232$

$053,-5.47 \mathrm{e}+1,4.00 \mathrm{E}+1,4.00 \mathrm{E}+2, \mathrm{~Pa}-231$

$121,-4.00 \mathrm{e}+1,7.00 \mathrm{E}-1,2.00 \mathrm{E}+1, \mathrm{U}-232$

$054,-4.00 \mathrm{e}+1,7.00 \mathrm{E}-1,2.00 \mathrm{E}+1, \mathrm{U}-233$

$038,-4.00 \mathrm{e}+1,7.00 \mathrm{E}-1,2.00 \mathrm{E}+1, \mathrm{U}-234$

$039,-4.00 \mathrm{e}+1,7.00 \mathrm{E}-1,2.00 \mathrm{E}+1, \mathrm{U}-235$

$040,-4.00 e+1,7.00 \mathrm{E}-1,2.00 \mathrm{E}+1, \mathrm{U}-236$

$041,-4.00 \mathrm{e}+1,7.00 \mathrm{E}-1,2.00 \mathrm{E}+1, \quad \mathrm{U}-238$

$042,-5.56 e+1,4.00 E+0,4.00 E+1, \quad N p-237$

$043,-5.76 \mathrm{e}+1,4.00 \mathrm{E}+0,4.00 \mathrm{E}+1, \quad \mathrm{Pu}-238$

$044,-5.76 e+1,4.00 E+0,4.00 E+1, \quad$ Pu-239

$045,-5.76 \mathrm{e}+1,4.00 \mathrm{E}+0,4.00 \mathrm{E}+1, \quad \mathrm{Pu}-240$

$048,-5.76 \mathrm{e}+1,4.00 \mathrm{E}+0,4.00 \mathrm{E}+1, \mathrm{Am}-241$

$132,-5.76 \mathrm{e}+1,4.00 \mathrm{E}+0,4.00 \mathrm{E}+1, \mathrm{Cm}-243$

$050,-5.76 \mathrm{e}+1,4.00 \mathrm{E}+0,4.00 \mathrm{E}+1, \mathrm{Cm}-244$

$020,-1.99 \mathrm{E}-1,0.00 \mathrm{E}+0,1.99 \mathrm{E}-1, \quad \mathrm{I}-129$

$025,-8.00 \mathrm{E}+2,8.00 \mathrm{E}+1,8.00 \mathrm{E}+2, \mathrm{Be}-10$

-- Input File: UPTAKE.DAT

$0.5, \quad 0.2, \quad 1.89$

$0.67, \quad 0.65, \quad 2.1 \mathrm{E}-3,438 ., 438$.

$0.0,2160 ., 24 ., \quad 1440 ., \quad 1 ., 0.83$

50., 6., $48 ., \quad 480 ., 48$.

$.05,0.0008,60 ., \quad 8 ., \quad 50$.

14., 176., 110., $\quad 0 ., \quad 95 ., \quad 730 ., 6.9$

$\mathrm{H}-3 \quad .25,4.8 \mathrm{E}+0,4.8 \mathrm{E}-1,1.0 \mathrm{E}-2, \quad 0 ., 1.2 \mathrm{E}-2,9.0 \mathrm{E}-1$

$\mathrm{C}-14 \quad .25,5.5 \mathrm{E}+0,5.5 \mathrm{E}-1,1.2 \mathrm{E}-2,2,0 ., 3.1 \mathrm{E}-2,4.6 \mathrm{E}+3$

$\mathrm{K}-40 \quad .25,3.7 \mathrm{E}-1,3.7 \mathrm{E}-2,1.0 \mathrm{E}-2,2,0 ., 1.2 \mathrm{E}-2,1.0 \mathrm{E}+3$

CO-57 .25, 9.4E-3, 9.4E-4, 1.0E-3, 0., 1.3E-2, 5.0E+1

CO-60 $25,9.4 \mathrm{E}-3,9.4 \mathrm{E}-4,1.0 \mathrm{E}-3,3,0 ., 1.3 \mathrm{E}-2,5.0 \mathrm{E}+1$

Sr-90 $\quad .25,1.7 \mathrm{E}-2,1.7 \mathrm{E}-3,8.0 \mathrm{E}-4,40.6 .0 \mathrm{E}-4,3.0 \mathrm{E}+1$

$\mathrm{Nb}-95 \quad .25,9.4 \mathrm{E}-3,9.4 \mathrm{E}-4,2.5 \mathrm{E}-3, \quad 0 ., 2.8 \mathrm{E}-1,3.0 \mathrm{E}+4$ 

TC -99
Cs-134
Cs -137
Ba-133
Eu-152
Eu-154
Eu-155
Ra-226
Th-228
Th-229
Th-230
Th-232
$\mathrm{Pa}-231$
U-232
$\mathrm{U}-233$
$\mathrm{U}-234$
$\mathrm{U}-235$
$\mathrm{U}-236$
$\mathrm{U}-238$
$\mathrm{Np}-237$
Pu-238
Pu-239
$\mathrm{Pu}-240$
Am- 241
$\mathrm{Cm}-243$
Cm- 244
I- 129
$\mathrm{Be}-10$

$.25,2.5 \mathrm{E}-1,2.5 \mathrm{E}-2,1.0 \mathrm{E}-3$, $.25,1.0 \mathrm{E}-2,1.0 \mathrm{E}-3,1.2 \mathrm{E}-2$, $.25,1.0 \mathrm{E}-2,1.0 \mathrm{E}-3,1.2 \mathrm{E}-2$ $.25,5.0 \mathrm{E}-3,5.0 \mathrm{E}-4,4.0 \mathrm{E}-4$, $.25,2.5 E-3,2.5 E-4,5.0 E-6$, $.25,2.5 \mathrm{E}-3,2.5 \mathrm{E}-4,5.0 \mathrm{E}-6$, $.25,2.5 E-3,2.5 E-4,5.0 E-6$, $.25,3.1 \mathrm{E}-4,3.1 \mathrm{E}-5,8.0 \mathrm{E}-3$, $.25,4.2 \mathrm{E}-3,4.2 \mathrm{E}-4,5.0 \mathrm{E}-6$, $.25,4.2 \mathrm{E}-3,4.2 \mathrm{E}-4,5.0 \mathrm{E}-6$, $.25,4.2 \mathrm{E}-3,4.2 \mathrm{E}-4,5.0 \mathrm{E}-6$, $.25,4.2 \mathrm{E}-3,4.2 \mathrm{E}-4,5.0 \mathrm{E}-6$, $.25,2.5 E-3,2.5 E-4,5.0 E-6$, $.25,2.5 E-3,2.5 E-4,5.0 E-4$, $.25,2.5 \mathrm{E}-3,2.5 \mathrm{E}-4,5.0 \mathrm{E}-4$, $.25,2.5 E-3,2.5 E-4,5.0 E-4$, $.25,2.5 E-3,2.5 E-4,5.0 E-4$, $.25,2.5 E-3,2.5 E-4,5.0 E-4$, $.25,2.5 \mathrm{E}-3,2.5 \mathrm{E}-4,5.0 \mathrm{E}-4$, $.25,2.5 E-3,2.5 E-4,5.0 E-6$, $.25,2.5 E-4,2.5 E-5,2.0 E-6$, $.25,2.5 \mathrm{E}-4,2.5 \mathrm{E}-5,2.0 \mathrm{E}-6$, $.25,2.5 E-4,2.5 E-5,2.0 E-6$, $.25,2.5 E-4,2.5 E-5,5.0 E-6$, $.25,2.5 E-3,2.5 E-4,5.0 E-6$, $.25,2.5 E-3,2.5 E-4,5.0 E-6$, $.25,2.0 \mathrm{E}-2,2.0 \mathrm{E}-3,7.0 \mathrm{E}-3$, $.25,1.0 \mathrm{E}-2,2.5 \mathrm{E}-3,9.0 \mathrm{E}-7$,
$0.1 .0 E-4,1.5 E+1$

$0 ., 4.0 E-3,2.0 E+3$

$0.4 .0 E-3,2.0 E+3$

$0 ., 3.2 \mathrm{E}-3,4.0 \mathrm{E}+0$

$0 ., 4.8 \mathrm{E}-3,2.5 \mathrm{E}+1$

$0 ., 4.8 \mathrm{E}-3,2.5 \mathrm{E}+1$

$0 ., 4.8 \mathrm{E}-3,2.5 \mathrm{E}+1$

$0 ., 3.4 \mathrm{E}-2,5.0 \mathrm{E}+1$

$0 ., 2.0 \mathrm{E}-4,3.0 \mathrm{E}+1$

$0 ., 2.0 \mathrm{E}-4,3.0 \mathrm{E}+1$

$0 ., 2.0 \mathrm{E}-4,3.0 \mathrm{E}+1$

$0 ., 2.0 E-4,3.0 E+1$

$0 ., 8.0 \mathrm{E}+2,1.1 \mathrm{E}+1$

$0 ., 3.4 \mathrm{E}-4,2.0 \mathrm{E}+0$

$0 ., 3.4 \mathrm{E}-4,2.0 \mathrm{E}+0$

$0 ., 3.4 \mathrm{E}-4,2.0 \mathrm{E}+0$

$0 ., 3.4 \mathrm{E}-4,2.0 \mathrm{E}+0$

$0 ., 3.4 \mathrm{E}-4,2.0 \mathrm{E}+0$

$0 ., 3.4 \mathrm{E}-4,2.0 \mathrm{E}+0$

$0 ., 2.0 \mathrm{E}-4,1.0 \mathrm{E}+1$

$0 ., 1.4 \mathrm{E}-5,3.5 \mathrm{E}+0$

$0 ., 1.4 \mathrm{E}-5,3.5 \mathrm{E}+0$

$0 ., 1.4 E-5,3.5 E+0$

$0 ., 2.0 E-4,2.5 E+1$

$0 ., 2.0 \mathrm{E}-4,2.5 \mathrm{E}+1$

$0 ., 2.0 \mathrm{E}-4,2.5 \mathrm{E}+1$

$0 ., 1.0 \mathrm{E}-2,4.0 \mathrm{E}+1$

$0 ., 1.0 \mathrm{E}-2,1.0 \mathrm{E}+2$ 
TOTAL EQUIVALENT UPTAKE FACTORS FOR PATHRAE

\begin{tabular}{|c|c|c|c|c|c|c|}
\hline & $\mathrm{UT}(\mathrm{J}, 1)$ & $\mathrm{UT}(\mathrm{J}, 2)$ & $\mathrm{UT}(\mathrm{J}, 3)$ & $\operatorname{UT}(\mathrm{J}, 4)$ & $\mathrm{UT}(\mathrm{J}, 5)$ & $\mathrm{UT}(\mathrm{J}, 6)$ \\
\hline & RIVER & WELL & EROSION & BATHTUB & SPILLAGE & FOOD \\
\hline$[D E$ & $L / Y R$ & $\mathrm{~L} / \mathrm{YR}$ & $\mathrm{L} / \mathrm{YR}$ & $\mathrm{L} / \mathrm{YR}$ & $L / Y R$ & $\mathrm{KG} / \mathrm{YR}$ \\
\hline $\mathrm{H}-3$ & $166 \mathrm{E}+03$ & $1.166 \mathrm{E}+03$ & 1. $172 \mathrm{E}+03$ & $1.172 \mathrm{E}+03$ & $1.172 \mathrm{E}+03$ & $00 \mathrm{E}+00$ \\
\hline$C-14$ & $9.564 \mathrm{E}+02$ & $9.564 \mathrm{E}+02$ & $3.270 \mathrm{E}+04$ & $3.270 E+04$ & $3.270 E+04$ & $0.000 \mathrm{E}+00$ \\
\hline$K-40$ & $.639 E+02$ & $8.639 \mathrm{E}+02$ & $7.764 \mathrm{E}+03$ & $7.765 \mathrm{E}+03$ & $7.765 E+03$ & $1.308 \mathrm{E}+01$ \\
\hline Co- 57 & $8.023 E+02$ & $8.023 \mathrm{E}+02$ & $1.146 \mathrm{E}+03$ & $1.146 \mathrm{E}+03$ & $1.146 \mathrm{E}+03$ & $1.933 \mathrm{E}-01$ \\
\hline Co- 60 & $8.055 \mathrm{E}+02$ & $8.055 \mathrm{E}+02$ & $1.150 \mathrm{E}+03$ & $1.150 \mathrm{E}+03$ & $1.150 \mathrm{E}+03$ & $2.120 \mathrm{E}-01$ \\
\hline $\operatorname{Sr}-90$ & $.414 \mathrm{E}+02$ & $7.414 \mathrm{E}+02$ & $9.484 E+02$ & $9.484 \mathrm{E}+02$ & $9.484 \mathrm{E}+02$ & $1.056 \mathrm{E}-01$ \\
\hline $\mathrm{Nb}-95$ & $.699 E+03$ & $1.699 \mathrm{E}+03$ & $2.007 E+05$ & $2.007 \mathrm{E}+05$ & $2.007 \mathrm{E}+05$ & $1.850 \mathrm{E}+00$ \\
\hline TC-99 & $7.403 \mathrm{E}+02$ & $7.403 \mathrm{E}+02$ & $8.438 \mathrm{E}+02$ & $8.438 \mathrm{E}+02$ & $8.438 \mathrm{E}+02$ & $1.469 \mathrm{E}+00$ \\
\hline Cs -134 & $8.365 E+02$ & $8.365 E+02$ & $1.461 \mathrm{E}+04$ & $1.461 \mathrm{E}+04$ & $1.461 \mathrm{E}+04$ & $2.702 E-01$ \\
\hline Cs -137 & $.371 E+02$ & $8.371 \mathrm{E}+02$ & $1.464 \mathrm{E}+04$ & $1.464 \mathrm{E}+04$ & $1.464 \mathrm{E}+04$ & $2.778 \mathrm{E}-01$ \\
\hline $\mathrm{Ba}-133$ & $7.518 \mathrm{E}+02$ & $7.518 \mathrm{E}+02$ & $7.794 \mathrm{E}+02$ & $7.794 \mathrm{E}+02$ & $7.794 \mathrm{E}+02$ & $4.490 \mathrm{E}-02$ \\
\hline Eu-152 & $7.572 E+02$ & $7.572 \mathrm{E}+02$ & $9.296 \mathrm{E}+02$ & $9.296 \mathrm{E}+02$ & $9.296 \mathrm{E}+02$ & $2.623 E-02$ \\
\hline Eu-154 & $7.571 E+02$ & $7.571 \mathrm{E}+02$ & $9.296 \mathrm{E}+02$ & $9.296 \mathrm{E}+02$ & $9.296 \mathrm{E}+02$ & $2.614 E-02$ \\
\hline Eu-155 & $7.570 \mathrm{E}+02$ & $7.570 \mathrm{E}+02$ & $9.294 \mathrm{E}+02$ & $9.294 \mathrm{E}+02$ & $9.294 \mathrm{E}+02$ & $2.595 \mathrm{E}-02$ \\
\hline Th-228 & $7.337 E+02$ & $7.337 \mathrm{E}+02$ & $9.403 E+02$ & $9.403 \mathrm{E}+02$ & $9.403 \mathrm{E}+02$ & $1.799 \mathrm{E}-02$ \\
\hline$U-232$ & $7.380 \mathrm{E}+02$ & $7.380 \mathrm{E}+02$ & $7.518 \mathrm{E}+02$ & $7.518 E+02$ & $7.518 \mathrm{E}+02$ & $1.356 \mathrm{E}-02$ \\
\hline $\mathrm{Cm}-243$ & $7.338 \mathrm{E}+02$ & $7.338 \mathrm{E}+02$ & $9.063 E+02$ & $9.063 \mathrm{E}+02$ & $9.063 \mathrm{E}+02$ & $1.118 \mathrm{E}-02$ \\
\hline$I-129$ & $8.327 E+02$ & $8.327 \mathrm{E}+02$ & $1.109 \mathrm{E}+03$ & $1.109 \mathrm{E}+03$ & $1.109 \mathrm{E}+03$ & $5.624 \mathrm{E}-01$ \\
\hline $\mathrm{Be}-10$ & $7.837 E+02$ & $7.837 \mathrm{E}+02$ & $1.474 \mathrm{E}+03$ & $1.474 \mathrm{E}+03$ & $1.474 \mathrm{E}+03$ & $2.267 \mathrm{E}-01$ \\
\hline Th-230 & $7.338 \mathrm{E}+02$ & $7.338 \mathrm{E}+02$ & $9.408 \mathrm{E}+02$ & $9.410 \mathrm{E}+02$ & $9.409 E+02$ & $1.884 \mathrm{E}-02$ \\
\hline Th-232 & $7.338 \mathrm{E}+02$ & $7.338 \mathrm{E}+02$ & $9.408 \mathrm{E}+02$ & $9.410 \mathrm{E}+02$ & 9.40 & $1.885 \Xi-02$ \\
\hline$U-234$ & $7.380 \mathrm{E}+02$ & $7.380 \mathrm{E}+02$ & $7.518 \mathrm{E}+02$ & $7.518 \mathrm{E}+02$ & 7.51 & $1.357 \mathrm{E}-02$ \\
\hline$U-235$ & $7.380 \mathrm{E}+02$ & $7.380 \mathrm{E}+02$ & $7.518 \mathrm{E}+02$ & $7.518 \mathrm{E}+02$ & $7.518 \mathrm{E}+02$ & $1.357 \mathrm{E}-02$ \\
\hline$U-236$ & $7.380 \mathrm{E}+02$ & $7.380 \mathrm{E}+02$ & $7.518 \mathrm{E}+02$ & $7.518 \mathrm{E}+02$ & $7.518 \mathrm{E}+02$ & $1.357 \mathrm{E}-02$ \\
\hline$U-238$ & $7.380 \mathrm{E}+02$ & $7.380 \mathrm{E}+02$ & $7.518 \mathrm{E}+02$ & $7.518 \mathrm{E}+02$ & $7.518 \mathrm{E}+02$ & $1.357 \mathrm{E}-02$ \\
\hline Np -237 & $7.338 \mathrm{E}+02$ & $7.338 \mathrm{E}+02$ & $8.028 \mathrm{E}+02$ & $8.028 \mathrm{E}+02$ & $8.028 \mathrm{E}+02$ & $1.122 \mathrm{E}-02$ \\
\hline $\mathrm{Pu}-238$ & $7.329 \mathrm{E}+02$ & $7.329 \mathrm{E}+02$ & $7.570 \mathrm{E}+02$ & $7.570 \mathrm{E}+02$ & $7.570 \mathrm{E}+02$ & $1.058 \mathrm{E}-03$ \\
\hline $\mathrm{Pu}-239$ & $7.329 E+02$ & $7.329 \mathrm{E}+02$ & $7.570 \mathrm{E}+02$ & $7.570 \mathrm{E}+02$ & $7.570 \mathrm{E}+02$ & $1.059 \mathrm{E}-03$ \\
\hline$P u-240$ & $7.329 \mathrm{E}+02$ & $7.329 \mathrm{E}+02$ & $7.570 \mathrm{E}+02$ & $7.570 \mathrm{E}+02$ & $7.570 \mathrm{E}+02$ & $1.059 E-03$ \\
\hline $\mathrm{Am}-241$ & $.338 \mathrm{E}+02$ & $7.338 \mathrm{E}+02$ & $9.063 \mathrm{E}+02$ & $9.063 \mathrm{E}+02$ & 9.06 & $1.121 \mathrm{E}-03$ \\
\hline $\mathrm{Cm}-244$ & $7.338 \mathrm{E}+02$ & $7.338 \mathrm{E}+02$ & $9.063 \mathrm{E}+02$ & $9.063 \mathrm{E}+02$ & $9.063 \mathrm{E}+02$ & $1.116 \mathrm{E}-02$ \\
\hline Th-229 & $7.338 \mathrm{E}+02$ & $7.338 \mathrm{E}+02$ & $9.408 \mathrm{E}+02$ & $9.408 \mathrm{E}+02$ & $9.409 \mathrm{E}+02$ & $1.884 \mathrm{E}-02$ \\
\hline
\end{tabular}


THERE ARE 80 ISOTOPES IN THE DOSE FACTOR LIBRARY NUMBER OF TIMES FOR CALCULATION IS 2

YEARS TO BE CALCULATED ARE ...

1000.00100000 .00

THERE ARE 35 ISOTOPES IN THE INVENTORY FILE

THE VALUE OF IFLAG IS 0

NUMBER OF PATHWAYS IS 5

\section{PATHWAY}

TYPE OF USAGE FOR UPTAKE FACTORS

1 GROUNDWATER TO RIVER

$0 \quad 3 X, I 2,2 X, A 22,6 X, I 2)) \square \square$

$0 \quad 3 \mathrm{X}, \mathrm{I} 2,2 \mathrm{X}, \mathrm{A} 22,6 \mathrm{X}, \mathrm{I2})) \square \square$

$0 \quad 3 X, I 2,2 X, A 22,6 X, I 2)) \square \square \quad 0$

TIME OF OPERATION OF WASTE FACILITY IN YEARS LENGTH OF REPOSITORY (M) WIDTH OF REPOSITORY (M)

RIVER FLOW RATE (M**3/YR)

STREAM FLOW RATE $(M * * 3 / Y R)$

DISTANCE TO RIVER (M)

OPERATIONAL SPILIAGE FRACTION

DENSITY OF AQUIFER (KG/M**3)

LONGITUDINAL DISPERSIVITY (M)

LATERAL DISPERSION COEFFICIENT - Y AXIS $(M * * 2 / Y R)$

NUMBER OF MESH POINTS FOR DISPERSION CALCULATION

0.

166 .

525 .

$2.23 E+05$

1. $00 \mathrm{E}+00$

74 .

$0.00 \mathrm{E}+00$

1800 .

6. $00 \mathrm{E}+00$

$0.00 \mathrm{E}+00$

20 
FLAG FOR GAMMA PATHWAY OPTIONS

FLAG FOR GAMMA BUILDUP CALCULATION

FLAG FOR ATMOSPHERIC PATHWAY

COVER THICKNESS OVER WASTE (M)

THICKNESS OF WASTE IN PITS (M)

TOTAL WASTE VOLUME $(\mathrm{M} * * 3)$

DISTANCE TO WELL - - X COORDINATE (M)

DISTANCE TO WELL - - Y COORDINATE (M)

DENSITY OF WASTE $(\mathrm{KG} / \mathrm{M} * * 3)$

FRACTION OF FOOD CONSUMED THAT IS GROWN ON SITE

FRACTION OF YEAR SPENT IN DIRECT RADIATION FIELD

DEPTH OF PLANT ROOT ZONE (M)

AREAL DENSITY OF PLANTS $(\mathrm{KG} / \mathrm{M} * * 2)$

AVERAGE DUST LOADING IN AIR (KG/M**3)

ANNUAL ADULT BREATHING RATE $(M * * 3 / Y R)$

FRACTION OF YEAR EXPOSED TO DUST

CANISTER LIFETTME (YEARS)

INVENTORY SCALING FACTOR

HEIGHT OF ROOMS IN RECLAIMER HOUSE (CM)

AIR CHANGE RATE IN RECLAIMER HOUSE (CHANGES/SEC)

RADON EMANATING POWER OF THE WASTE

DIFFUSION COEFF. OF RADON IN WASTE (CM**2/SEC)

DIFFUSION COEFF. OF RN IN CONCRETE (CM**2/SEC)

THICKNESS OF CONCRETE SLAB FLOOR (CM)

DIFFUSION COEFF. OF RADON IN COVER (CM**2/SEC)

ATMOSPHERIC STABILITY CLASS

AVERAGE WIND SPEED (M/S)

FRACTION OF TIME WIND BLOWS TOWARD RECEPTOR

RECEPTOR DISTANCE FOR ATMOSPHERIC PATHWAY (M)

DUST RESUSPENSION RATE FOR OFFSTTE TRANSPORT $(M * * 3 / S)$

DEPOSITION VELOCITY (M/S)

STACK HETGHT (M)

STACK INSIDE DIAMETER (M)

2
0
0
4.00
15.30
$1.324 \mathrm{E}+06$
-1.
0.
1600.
.400
.705
.900
1.000
$1.00 \mathrm{E}-07$
8000.
.705
0.
$1.00 \mathrm{E}+00$
240.
$5.56 \mathrm{E}-04$
$2.20 \mathrm{E}-01$
$2.00 \mathrm{E}-02$
$3.00 \mathrm{E}-04$
20.0
$1.00 \mathrm{E}-02$
4
6.30
.2300
.0
$1.10 \mathrm{E}-06$
.0100
.0
.00


STACK GAS VELOCITY (M/S)

HEAT EMISSION RATE FROM BURNING (CAL/S)

DECAY CHAIN FLAGS

FLAG FOR INPUT SUMMARY PRINTOUT

FLAG FOR DIRECTION OF TRENCH FILLING

FLAG FOR GROUNDWATER PATHWAY OPTIONS

AMOUNT OF WATER PERCOLATING THROUGH WASTE ANNUALLY (M)

DEGREE OF SOIL SATURATION

RESIDUAL SOIL SATURATION

PERMEABILITY OF VERTICAL ZONE (M/YR)

SOIL NUMBER

POROSITY OF AQUTFER

POROSITY OF UNSATURATED ZONE

DISTANCE FROM AQUIFER TO WASTE (M)

AVERAGE VERTICAL GROUNDWATER VELOCITY (M/YR)

HORIZONTAL VELOCITY OF AQUIFER (M/YR)

LENGTH OF PERFORATED WELL CASING (M)

SURFACE EROSION RATE (M/YR)

LEACH RATE SCALING FACTOR

ANNUAL RUNOFF OF PRECIPITATION (M)

$0.00 E+00$

0

1

1

9. $10 \mathrm{E}-03$

1.000

.000

.32

.000

.04

.25

4.6

$2.50 \mathrm{E}-02$

4.6

10.000

1. $000 \mathrm{E}-05$

1. $000 \mathrm{E}+00$

$0.00 E+00$ 
***** PEAK CONCENTRATIONS AND TIMES FOR PATHWAY

$* * * * *$ RIVER AT $73.9 \mathrm{M} * * * * *$

NUCLIDE
H-3
$\mathrm{C}-14$
$\mathrm{~K}-40$
$\mathrm{Co}-57$
$\mathrm{CO}-60$
$\mathrm{TC}-99$
$\mathrm{Cs}-134$
$\mathrm{Cs}-137$
$\mathrm{Th}-228$
$\mathrm{I}-129$
$\mathrm{Be}-10$
$\mathrm{Th}-230$
$\mathrm{Th}-232$
$\mathrm{U}-234$
$\mathrm{U}-235$
$\mathrm{U}-236$
$\mathrm{U}-238$
$\mathrm{~Np}-237$
$\mathrm{Pu}-239$
$\mathrm{Pu}-240$
$\mathrm{Am}-241$
$\mathrm{Th}-229$
$\mathrm{~Pa}-231$
$\mathrm{U}-233$
$\mathrm{Ra}-226$

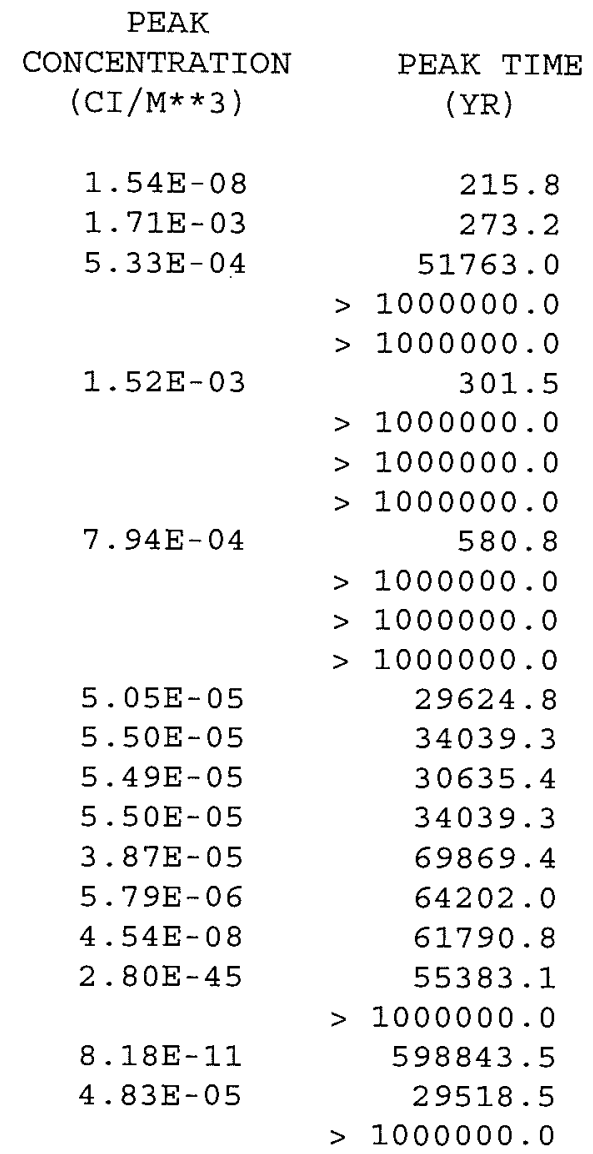

AVERAGE DOSE

AT PEAK TIME

(MREM/YR)

$\begin{array}{ll}1.15 \mathrm{E}-03 & 3.21 \mathrm{E}-10 \\ 3.44 \mathrm{E}+03 & 9.63 \mathrm{E}-04 \\ 8.75 \mathrm{E}+03 & 2.45 \mathrm{E}-03\end{array}$

$1.69 \mathrm{E}+03$

4. $74 \mathrm{E}-04$

$1.85 \mathrm{E}+05$

$5.19 \mathrm{E}-02$

2. $95 E-03$

3. $03 E-03$

3. $05 \mathrm{E}-03$

3. $07 \mathrm{E}-03$

3. $50 \mathrm{E}-02$

4. $16 \mathrm{E}-03$

$3.26 \mathrm{E}-05$

$2.07 \mathrm{E}-42$

$2.33 E-03$

$2.88 \mathrm{E}-03$ 
PATHWAY 1

GROUNDWATER TO RIVER

$\star * * * * * * * *$ NUCLIDE DOSES (mrem/yr) $* * * * * * * *$

$\begin{array}{lcc}\text { NUCLIDE/TIME } & 1000 & 100000 \\ \mathrm{H}-3 & 0.0 \mathrm{E}+00 & 0.0 \mathrm{E}+00 \\ \mathrm{C}-14 & 3.2 \mathrm{E}+03 & 0.0 \mathrm{E}+00 \\ \mathrm{~K}-40 & 0.0 \mathrm{E}+00 & 0.0 \mathrm{E}+00 \\ \mathrm{CO}-57 & 0.0 \mathrm{E}+00 & 0.0 \mathrm{E}+00 \\ \mathrm{CO}-60 & 0.0 \mathrm{E}+00 & 0.0 \mathrm{E}+00 \\ \mathrm{Sr}-90 & 0.0 \mathrm{E}+00 & 0.0 \mathrm{E}+00 \\ \mathrm{Nb}-95 & 0.0 \mathrm{E}+00 & 0.0 \mathrm{E}+00 \\ \mathrm{TC}-99 & 1.7 \mathrm{E}+03 & 0.0 \mathrm{E}+00 \\ \mathrm{C} s-134 & 0.0 \mathrm{E}+00 & 0.0 \mathrm{E}+00 \\ \mathrm{Cs}-137 & 0.0 \mathrm{E}+00 & 0.0 \mathrm{E}+00 \\ \mathrm{Ba}-133 & 0.0 \mathrm{E}+00 & 0.0 \mathrm{E}+00 \\ \mathrm{Eu}-152 & 0.0 \mathrm{E}+00 & 0.0 \mathrm{E}+00 \\ \mathrm{Eu}-154 & 0.0 \mathrm{E}+00 & 0.0 \mathrm{E}+00 \\ \mathrm{Eu}-155 & 0.0 \mathrm{E}+00 & 0.0 \mathrm{E}+00 \\ \mathrm{Th}-228 & 0.0 \mathrm{E}+00 & 0.0 \mathrm{E}+00 \\ \mathrm{U}-232 & 0.0 \mathrm{E}+00 & 0.0 \mathrm{E}+00 \\ \mathrm{Cm}-243 & 0.0 \mathrm{E}+00 & 0.0 \mathrm{E}+00 \\ \mathrm{I}-129 & 1.9 \mathrm{E}+05 & 0.0 \mathrm{E}+00 \\ \mathrm{Be}-10 & 0.0 \mathrm{E}+00 & 0.0 \mathrm{E}+00 \\ \mathrm{Th}-230 & 0.0 \mathrm{E}+00 & 0.0 \mathrm{E}+00 \\ \mathrm{Th}-232 & 0.0 \mathrm{E}+00 & 0.0 \mathrm{E}+00 \\ \mathrm{U}-234 & 0.0 \mathrm{E}+00 & 8.6 \mathrm{E}+03 \\ \mathrm{U}-235 & 0.0 \mathrm{E}+00 & 1.1 \mathrm{E}+04 \\ \mathrm{U}-236 & 0.0 \mathrm{E}+00 & 1.1 \mathrm{E}+04 \\ \mathrm{U}-238 & 0.0 \mathrm{E}+00 & 1.1 \mathrm{E}+04 \\ \mathrm{~Np}-237 & 0.0 \mathrm{E}+00 & 1.2 \mathrm{E}+05 \\ \mathrm{Pu}-238 & 0.0 \mathrm{E}+00 & 0.0 \mathrm{E}+00 \\ \mathrm{Pu}-239 & 0.0 \mathrm{E}+00 & 5.5 \mathrm{E}+03 \\ \mathrm{Pu}-240 & 0.0 \mathrm{E}+00 & 2.4 \mathrm{E}+00 \\ & & \end{array}$




$\begin{array}{lll}\mathrm{Am}-241 & 0.0 \mathrm{E}+00 & 0.0 \mathrm{E}+00 \\ \mathrm{Cm}-244 & 0.0 \mathrm{E}+00 & 0.0 \mathrm{E}+00 \\ \mathrm{Th}-229 & 0.0 \mathrm{E}+00 & 0.0 \mathrm{E}+00 \\ \mathrm{~Pa}-231 & 0.0 \mathrm{E}+00 & 0.0 \mathrm{E}+00 \\ \mathrm{U}-233 & 0.0 \mathrm{E}+00 & 7.6 \mathrm{E}+03 \\ \mathrm{Ra}-226 & 0.0 \mathrm{E}+00 & 0.0 \mathrm{E}+00\end{array}$

NUCLIDE CONCENTRATIONS IN RIVER ( $\mathrm{Ci} / \mathrm{m} * * 3)$

$\begin{array}{lcc}\text { NUCLIDE/TIME } & 1000 & 100000 \\ \mathrm{H}-3 & 0.0 \mathrm{E}+00 & 0.0 \mathrm{E}+00 \\ \mathrm{C}-14 & 1.6 \mathrm{E}-03 & 0.0 \mathrm{E}+00 \\ \mathrm{~K}-40 & 0.0 \mathrm{E}+00 & 0.0 \mathrm{E}+00 \\ \mathrm{CO}-57 & 0.0 \mathrm{E}+00 & 0.0 \mathrm{E}+00 \\ \mathrm{CO}-60 & 0.0 \mathrm{E}+00 & 0.0 \mathrm{E}+00 \\ \mathrm{~S}-90 & 0.0 \mathrm{E}+00 & 0.0 \mathrm{E}+00 \\ \mathrm{Nb}-95 & 0.0 \mathrm{E}+00 & 0.0 \mathrm{E}+00 \\ \mathrm{TC}-99 & 1.5 \mathrm{E}-03 & 0.0 \mathrm{E}+00 \\ \mathrm{CS}-134 & 0.0 \mathrm{E}+00 & 0.0 \mathrm{E}+00 \\ \mathrm{CS}-137 & 0.0 \mathrm{E}+00 & 0.0 \mathrm{E}+00 \\ \mathrm{Ba}-133 & 0.0 \mathrm{E}+00 & 0.0 \mathrm{E}+00 \\ \mathrm{Eu}-152 & 0.0 \mathrm{E}+00 & 0.0 \mathrm{E}+00 \\ \mathrm{Eu}-154 & 0.0 \mathrm{E}+00 & 0.0 \mathrm{E}+00 \\ \mathrm{Eu}-155 & 0.0 \mathrm{E}+00 & 0.0 \mathrm{E}+00 \\ \mathrm{Th}-228 & 0.0 \mathrm{E}+00 & 0.0 \mathrm{E}+00 \\ \mathrm{U}-232 & 0.0 \mathrm{E}+00 & 0.0 \mathrm{E}+00 \\ \mathrm{Cm}-243 & 0.0 \mathrm{E}+00 & 0.0 \mathrm{E}+00 \\ \mathrm{I}-129 & 7.9 \mathrm{E}-04 & 0.0 \mathrm{E}+00 \\ \mathrm{Be}-10 & 0.0 \mathrm{E}+00 & 0.0 \mathrm{E}+00 \\ \mathrm{Th}-230 & 0.0 \mathrm{E}+00 & 0.0 \mathrm{E}+00 \\ \mathrm{Th}-232 & 0.0 \mathrm{E}+00 & 0.0 \mathrm{E}+00 \\ \mathrm{U}-234 & 0.0 \mathrm{E}+00 & 4.1 \mathrm{E}-05 \\ \mathrm{U}-235 & 0.0 \mathrm{E}+00 & 5.5 \mathrm{E}-05 \\ \mathrm{U}-236 & 0.0 \mathrm{E}+00 & 5.5 \mathrm{E}-05 \\ \mathrm{U}-238 & 0.0 \mathrm{E}+00 & 5.5 \mathrm{E}-05 \\ \mathrm{~Np}-237 & 0.0 \mathrm{E}+00 & 3.8 \mathrm{E}-05 \\ \mathrm{Pu}-238 & 0.0 \mathrm{E}+00 & 0.0 \mathrm{E}+00\end{array}$


Pu-239

$\mathrm{Pu}-240$

Am-24I

$\mathrm{Cm}-244$

Th-229

$\mathrm{Pa}-231$

$\mathrm{U}-233$

Ra-226
$0.0 E+00$

$0.0 E+00$

$0.0 \mathrm{E}+00$

$0.0 \mathrm{E}+00$

$0.0 E+00$

$0.0 E+00$

$0.0 \mathrm{E}+00$

$0.0 \mathrm{E}+00$

2. $2 E-06$

. $5 \mathrm{E}-10$

$0.0 E+00$

$0.0 E+00$

$0.0 E+00$

$0.0 \mathrm{E}+00$

3. $6 \mathrm{E}-05$

$0.0 \mathrm{E}+00$ 
PATHRAE-HAZ (PC) Version 2.2d February 1995

Date: $\quad 8-12-2009$

Time: $10: 36: 31$

W. A. C. - August, 2009 New Proposed Cell 1-5 EMWMF

\section{CONTAMINANT}

Acenaphthene

Acetone

Anthracene

Aluminum

Antimony

Arochlors -1242

Arochlors -1254

Arochlors-1248

Arochlors-1260

Arsenic

Barium

Benzene

BenzoAanthracene

Beryllium

Bis2ethylhexapht

Butylbenzylphtha

Cadmium

Calcium

Carbazole

Carbontetchl

chloroform

Chromium III

Chromium VI
TOTAL EQUIVALENT UPTAKE FACTORS FOR PATHRAE

$\begin{array}{cccccc}\mathrm{UT}(\mathrm{J}, 1) & \mathrm{UT}(\mathrm{J}, 2) & \mathrm{UT}(\mathrm{J}, 3) & \mathrm{UT}(\mathrm{J}, 4) & \mathrm{UT}(\mathrm{J}, 5) & \mathrm{UT}(\mathrm{J}, 6) \\ \text { RIVER } & \text { WELL } & \text { EROSION } & \text { BATHTUB } & \text { SPILLAGE } & \text { FOOD } \\ \text { L/YR } & \mathrm{L} / \mathrm{YR} & \mathrm{L} / \mathrm{YR} & \mathrm{L} / \mathrm{YR} & \mathrm{L} / \mathrm{YR} & \mathrm{KG} / \mathrm{YR}\end{array}$

$\begin{array}{llllll}7.365 E+02 & 7.365 E+02 & 8.326 E+03 & 8.327 E+03 & 8.327 E+03 & 6.144 E-01\end{array}$ $\begin{array}{llllll}7.328 \mathrm{E}+02 & 7.328 \mathrm{E}+02 & 7.328 \mathrm{E}+02 & 7.328 \mathrm{E}+02 & 7.328 \mathrm{E}+02 & 5.480 \mathrm{E}+01\end{array}$ $\begin{array}{llllll}7.374 E+02 & 7.374 E+02 & 9.707 E+03 & 9.708 E+03 & 9.708 E+03 & 2.612 E-01\end{array}$ $\begin{array}{lllllll}7.418 E+02 & 7.418 E+02 & 7.418 E+02 & 7.419 E+02 & 7.419 E+02 & 2.601 E-02\end{array}$ $\begin{array}{llllll}7.332 E+02 & 7.332 E+02 & 1.423 E+03 & 1.423 E+03 & 1.423 E+03 & 2.153 E-01\end{array}$ $\begin{array}{lllllll}7.351 E+02 & 7.351 E+02 & 6.048 E+03 & 6.048 E+03 & 6.048 E+03 & 7.641 E-01\end{array}$ $9.154 \mathrm{E}+02 \quad 9.154 \mathrm{E}+02 \quad 1.458 \mathrm{E}+05 \quad 1.458 \mathrm{E}+05 \quad 1.458 \mathrm{E}+05 \quad 6.409 \mathrm{E}-01$ $8.493 \mathrm{E}+02 \quad 8.493 \mathrm{E}+02 \quad 1.043 \mathrm{E}+05 \quad 1.044 \mathrm{E}+05 \quad 1.044 \mathrm{E}+05 \quad 5.277 \mathrm{E}+00$ $3.005 \mathrm{E}+03 \quad 3.005 \mathrm{E}+03 \quad 1.038 \mathrm{E}+06 \quad 1.038 \mathrm{E}+06 \quad 1.038 \mathrm{E}+06 \quad 1.638 \mathrm{E}+00$

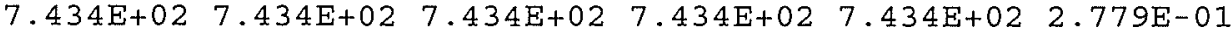
$7.372 \mathrm{E}+02 \quad 7.372 \mathrm{E}+02 \quad 7.648 \mathrm{E}+02 \quad 7.649 \mathrm{E}+02 \quad 7.649 \mathrm{E}+02 \quad 5.213 \mathrm{E}-01$ $\begin{array}{llllll}7.328 E+02 & 7.328 E+02 & 7.328 E+02 & 7.328 E+02 & 7.328 E+02 & 2.450 E+00\end{array}$ $\begin{array}{llllll}8.270 E+02 & 8.270 E+02 & 9.053 E+04 & 9.055 E+04 & 9.053 E+04 & 5.224 E-01\end{array}$ $\begin{array}{llllll}7.379 \mathrm{E}+02 & 7.379 \mathrm{E}+02 & 1.428 \mathrm{E}+03 & 1.428 \mathrm{E}+03 & 1.428 \mathrm{E}+03 & 5.537 \mathrm{E}-02\end{array}$ $\begin{array}{llllll}7.474 E+02 & 7.474 E+02 & 2.214 E+04 & 2.216 E+04 & 2.214 E+04 & 4.300 E-01\end{array}$ $\begin{array}{lllllll}7.738 \mathrm{E}+02 & 7.738 \mathrm{E}+02 & 2.214 \mathrm{E}+04 & 2.216 \mathrm{E}+04 & 2.214 \mathrm{E}+04 & 4.300 \mathrm{E}-01\end{array}$ $\begin{array}{llllll}7.418 E+02 & 7.418 E+02 & 2.122 E+03 & 2.127 E+03 & 2.126 E+03 & 3.450 E+00\end{array}$ $8.102 \mathrm{E}+02 \quad 8.102 \mathrm{E}+02 \quad 8.091 \mathrm{E}+02 \quad 8.102 \mathrm{E}+02 \quad 8.102 \mathrm{E}+02 \quad 7.822 \mathrm{E}-01$ $\begin{array}{llllll}7.340 \mathrm{E}+02 & 7.340 \mathrm{E}+02 & 3.839 \mathrm{E}+03 & 3.839 \mathrm{E}+03 & 3.839 \mathrm{E}+03 \quad 1.081 \mathrm{E}+00\end{array}$ $\begin{array}{llllll}7.329 \mathrm{E}+02 & 7.329 \mathrm{E}+02 & 7.329 \mathrm{E}+02 & 7.329 \mathrm{E}+02 & 7.329 \mathrm{E}+02 & 1.231 \mathrm{E}+00\end{array}$ $7.328 \mathrm{E}+02 \quad 7.328 \mathrm{E}+02 \quad 7.328 \mathrm{E}+02 \quad 7.328 \mathrm{E}+02 \quad 7.328 \mathrm{E}+02 \quad 2.955 \mathrm{E}+00$ $\begin{array}{llllll}7.787 \mathrm{E}+02 & 7.787 \mathrm{E}+02 & 2.159 \mathrm{E}+03 & 2.159 \mathrm{E}+03 & 2.159 \mathrm{E}+03 & 6.445 \mathrm{E}-01\end{array}$ $\begin{array}{llllll}7.787 E+02 & 7.787 E+02 & 2.159 E+03 & 2.160 E+03 & 2.160 E+03 & 6.445 E-01\end{array}$ 
Cobalt

Copper

Benzobflranthene

BenzoKflranthene

Benzoghiperylene

Benzo (a) pyrene

Dibenzoahanthrac

Dibenzofuran

Dieldrin

Dinbutylphthalat

Dinoctylphthalat

24-dinitrotoluen

Fluoranthene

Flourene

2-Hexanone

Indeno123cdpyren

Iron

Isophorone

Lead

Lithium

Magnesium

Manganese

Mercury

Methchloride

2Methylnaphthale

Naphthalene

Nickel

Nnitronpropyl

Pentachloropheno

Phenanthrene

Phenol

Potassium

Pyrene

Selenium

Silver

Sodium

strontium

Tetrachloroethen
$7.415 \mathrm{E}+02 \quad 7.415 \mathrm{E}+02 \quad 7.414 \mathrm{E}+02 \quad 7.415 \mathrm{E}+02 \quad 7.415 \mathrm{E}+02 \quad 5.922 \mathrm{E}-02$ $\begin{array}{llllll}7.891 E+02 & 7.891 E+02 & 3.527 E+05 & 3.527 E+05 & 3.527 E+05 & 1.471 E+01\end{array}$ $9.600 \mathrm{E}+02 \quad 9.600 \mathrm{E}+02 \quad 1.735 \mathrm{E}+05 \quad 1.735 \mathrm{E}+05 \quad 1.735 \mathrm{E}+05 \quad 6.631 \mathrm{E}-01$ $\begin{array}{lllllll}1.898 E+03 & 1.898 E+03 & 6.022 E+05 & 6.022 E+05 & 6.022 E+05 & 1.255 E+00\end{array}$ $\begin{array}{llllll}1.459 E+03 & 1.459 E+03 & 4.224 E+05 & 4.224 E+05 & 4.224 E+05 & 1.028 E+00\end{array}$ $9.600 \mathrm{E}+02 \quad 9.600 \mathrm{E}+02 \quad 1.735 \mathrm{E}+05 \quad 1.735 \mathrm{E}+05 \quad 1.735 \mathrm{E}+05 \quad 6.631 \mathrm{E}-01$ $\begin{array}{llllll}1.898 \mathrm{E}+03 & 1.898 \mathrm{E}+03 & 6.022 \mathrm{E}+05 & 6.023 \mathrm{E}+05 & 6.022 \mathrm{E}+05 & 1.255 \mathrm{E}+00\end{array}$ $\begin{array}{llllll}7.328 \mathrm{E}+02 & 7.328 \mathrm{E}+02 & 7.328 \mathrm{E}+02 & 7.328 \mathrm{E}+02 & 7.328 \mathrm{E}+02 & 0.000 \mathrm{E}+00\end{array}$ $\begin{array}{llllll}8.286 \mathrm{E}+02 & 8.286 \mathrm{E}+02 & 8.284 \mathrm{E}+02 & 8.287 \mathrm{E}+02 & 8.287 \mathrm{E}+02 & 2.459 \mathrm{E}+00\end{array}$ $\begin{array}{llllll}8.061 E+02 & 8.061 E+02 & 8.061 E+02 & 8.061 E+02 & 8.061 E+02 & 1.249 E-01\end{array}$ $\begin{array}{llllll}7.328 E+02 & 7.328 E+02 & 7.328 E+02 & 7.328 E+02 & 7.328 E+02 & 0.000 E+00\end{array}$ $\begin{array}{llllll}7.328 \mathrm{E}+02 & 7.328 \mathrm{E}+02 & 8.639 \mathrm{E}+02 & 8.639 \mathrm{E}+02 & 8.639 \mathrm{E}+02 & 1.097 \mathrm{E}+01\end{array}$ $\begin{array}{lllllll}7.474 \mathrm{E}+02 & 7.474 \mathrm{E}+02 & 2.214 \mathrm{E}+04 & 2.214 \mathrm{E}+04 & 2.214 \mathrm{E}+04 & 4.300 \mathrm{E}-01\end{array}$ $\begin{array}{lllllll}7.374 \mathrm{E}+02 & 7.374 \mathrm{E}+02 & 9.707 \mathrm{E}+03 & 9.708 \mathrm{E}+03 & 9.708 \mathrm{E}+03 & 5.888 \mathrm{E}-01\end{array}$ $\begin{array}{llllll}7.328 \mathrm{E}+02 & 7.328 \mathrm{E}+02 & 7.797 \mathrm{E}+02 & 7.797 \mathrm{E}+02 & 7.797 \mathrm{E}+02 & 2.488 \mathrm{E}+00\end{array}$ $\begin{array}{llllll}1.459 \mathrm{E}+03 & 1.459 \mathrm{E}+03 & 4.224 \mathrm{E}+05 & 4.224 \mathrm{E}+05 & 4.224 \mathrm{E}+05 & 1.028 \mathrm{E}+00\end{array}$ $\begin{array}{llllll}8.349 E+02 & 8.349 E+02 & 2.215 E+03 & 2.216 E+03 & 2.216 E+03 & 3.067 E-01\end{array}$ $\begin{array}{llllll}7.329 E+02 & 7.329 E+02 & 7.328 E+02 & 7.329 E+02 & 7.329 E+02 & 2.030 E+00\end{array}$ $\begin{array}{llllll}7.369 E+02 & 7.369 E+02 & 2.807 E+03 & 2.807 E+03 & 2.807 E+03 & 4.682 E-01\end{array}$ $\begin{array}{llllll}7.328 \mathrm{E}+02 & 7.328 \mathrm{E}+02 & 7.328 \mathrm{E}+02 & 7.328 \mathrm{E}+02 & 7.328 \mathrm{E}+02 & 0.000 \mathrm{E}+00\end{array}$ $8.097 \mathrm{E}+02 \quad 8.097 \mathrm{E}+02 \quad 7.863 \mathrm{E}+02 \quad 8.097 \mathrm{E}+02 \quad 8.082 \mathrm{E}+02 \quad 1.693 \mathrm{E}+01$ $\begin{array}{llllll}7.355 \mathrm{E}+02 & 7.355 \mathrm{E}+02 & 3.496 \mathrm{E}+03 & 3.498 \mathrm{E}+03 & 3.498 \mathrm{E}+03 & 3.346 \mathrm{E}+00\end{array}$

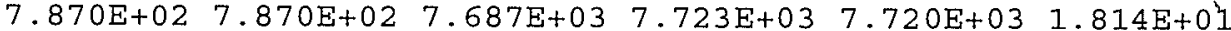
$7.328 \mathrm{E}+02 \quad 7.328 \mathrm{E}+02 \quad 7.328 \mathrm{E}+02 \quad 7.328 \mathrm{E}+02 \quad 7.328 \mathrm{E}+02 \quad 0.000 \mathrm{E}+00$

$\begin{array}{llllll}7.328 \mathrm{E}+02 & 7.328 \mathrm{E}+02 & 7.328 \mathrm{E}+02 & 7.328 \mathrm{E}+02 & 7.328 \mathrm{E}+02 & 0.000 \mathrm{E}+00\end{array}$ $\begin{array}{llllll}7.332 \mathrm{E}+02 & 7.332 \mathrm{E}+02 & 2.044 \mathrm{E}+03 & 2.044 \mathrm{E}+03 & 2.044 \mathrm{E}+03 & 1.981 \mathrm{E}+00\end{array}$ $\begin{array}{lllllll}8.702 E+02 & 8.702 E+02 & 1.560 E+03 & 1.582 E+03 & 1.578 E+03 & 6.350 E+00\end{array}$ $\begin{array}{llllll}7.328 \mathrm{E}+02 & 7.328 \mathrm{E}+02 & 7.797 \mathrm{E}+02 & 7.797 \mathrm{E}+02 & 7.797 \mathrm{E}+02 & 2.488 \mathrm{E}+01\end{array}$ $\begin{array}{llllll}7.635 \mathrm{E}+02 & 7.635 \mathrm{E}+02 & 7.630 \mathrm{E}+02 & 7.635 \mathrm{E}+02 & 7.634 \mathrm{E}+02 & 1.361 \mathrm{E}-01\end{array}$ $7.405 E+02 \quad 7.405 E+02 \quad 1.316 E+04 \quad 1.316 E+04 \quad 1.316 E+04 \quad 4.928 E-01$ $\begin{array}{llllll}7.328 \mathrm{E}+02 & 7.328 \mathrm{E}+02 & 7.887 \mathrm{E}+02 & 7.887 \mathrm{E}+02 & 7.887 \mathrm{E}+02 & 2.150 \mathrm{E}+01\end{array}$ $\begin{array}{lllllll}8.653 E+02 & 8.653 \mathrm{E}+02 & 7.764 \mathrm{E}+03 & 7.765 \mathrm{E}+03 & 7.765 \mathrm{E}+03 & 1.308 \mathrm{E}+01\end{array}$ $\begin{array}{llllll}7.474 \mathrm{E}+02 & 7.474 \mathrm{E}+02 & 2.214 \mathrm{E}+04 & 2.214 \mathrm{E}+04 & 2.214 \mathrm{E}+04 & 4.300 \mathrm{E}-01\end{array}$ $\begin{array}{llllll}1.312 \mathrm{E}+03 & 1.312 \mathrm{E}+03 & 1.312 \mathrm{E}+03 & 1.316 \mathrm{E}+03 & 1.316 \mathrm{E}+03 & 7.577 \mathrm{E}+01\end{array}$ $\begin{array}{llllll}7.484 E+02 & 7.484 E+02 & 7.829 E+02 & 7.949 E+02 & 7.941 E+02 & 8.253 E+00\end{array}$ $\begin{array}{llllll}1.253 E+03 & 1.253 E+03 & 1.390 E+03 & 1.391 E+03 & 1.391 E+03 & 2.686 E+01\end{array}$ $\begin{array}{llllll}7.941 E+02 & 7.941 E+02 & 7.931 E+02 & 7.941 E+02 & 7.941 E+02 & 2.096 E+01\end{array}$ $\begin{array}{llllll}7.329 \mathrm{E}+02 & 7.329 \mathrm{E}+02 & 7.329 \mathrm{E}+02 & 7.329 \mathrm{E}+02 & 7.329 \mathrm{E}+02 & 1.273 \mathrm{E}+00\end{array}$ 
Thallium

Tin

Toluene

Trichloroethene

$\mathrm{U}-232$

$\mathrm{U}-233$

$\mathrm{U}-234$

$\mathrm{U}-235$

$\mathrm{U}-236$

$\mathrm{U}-238$

Vanadium

zinc

zirconium

$12378 \mathrm{PeCDF}$

$23478 \mathrm{PeCDF}$

OCDD

OCDF

$1234678 \mathrm{HpCDD}$

$1234678 \mathrm{HpCDF}$

$123478 \mathrm{HXCDF}$

$12378 \mathrm{HxCDD}$

$123678 \mathrm{HxCDD}$

$123789 \mathrm{HxCDD}$

$2378 \mathrm{TCDD}$

$2378 \mathrm{TCDF}$

Hexachloroethene

124Trichlorb

Molybdenum

Strontium

Acenaphthylene

benzidine

Chlordane

MethylPropylB

124 TrimethylB

135TrimethylB

MethylethylB

ButylB

12DiMethylB
$7.328 \mathrm{E}+02 \quad 7.328 \mathrm{E}+02 \quad 7.328 \mathrm{E}+02 \quad 7.328 \mathrm{E}+02 \quad 7.328 \mathrm{E}+02 \quad 0.000 \mathrm{E}+00$ $\begin{array}{lllllll}7.907 \mathrm{E}+02 & 7.907 \mathrm{E}+02 & 2.149 \mathrm{E}+04 & 2.149 \mathrm{E}+04 & 2.149 \mathrm{E}+04 & 1.895 \mathrm{E}+01\end{array}$ $\begin{array}{llllll}7.330 \mathrm{E}+02 & 7.330 \mathrm{E}+02 & 7.329 \mathrm{E}+02 & 7.330 \mathrm{E}+02 & 7.330 \mathrm{E}+02 & 1.106 \mathrm{E}+00\end{array}$ $\begin{array}{llllll}7.329 \mathrm{E}+02 & 7.329 \mathrm{E}+02 & 7.329 \mathrm{E}+02 & 7.329 \mathrm{E}+02 & 7.329 \mathrm{E}+02 & 1.735 \mathrm{E}+00\end{array}$

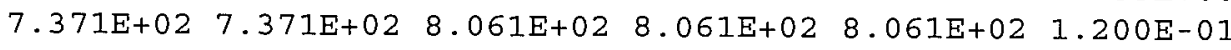
$\begin{array}{llllll}7.371 E+02 & 7.371 E+02 & 8.061 E+02 & 8.061 E+02 & 8.061 E+02 & 1.201 E-01\end{array}$

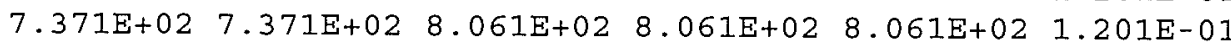
$\begin{array}{llllll}7.371 \mathrm{E}+02 & 7.371 \mathrm{E}+02 & 8.061 \mathrm{E}+02 & 8.061 \mathrm{E}+02 & 8.061 \mathrm{E}+02 & 1.201 \mathrm{E}-01\end{array}$ $\begin{array}{llllll}7.371 E+02 & 7.371 E+02 & 8.061 E+02 & 8.061 E+02 & 8.061 E+02 & 1.201 E-01\end{array}$ $\begin{array}{llllll}7.371 \mathrm{E}+02 & 7.371 \mathrm{E}+02 & 8.061 \mathrm{E}+02 & 8.061 \mathrm{E}+02 & 8.061 \mathrm{E}+02 & 1.201 \mathrm{E}-01\end{array}$

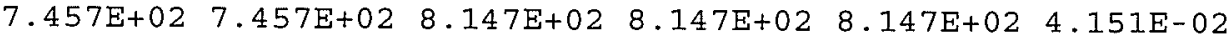
$\begin{array}{lllllll}1.312 \mathrm{E}+03 & 1.312 \mathrm{E}+03 & 8.212 \mathrm{E}+03 & 8.693 \mathrm{E}+03 & 8.619 \mathrm{E}+03 & 1.500 \mathrm{E}+02\end{array}$ $\begin{array}{llllll}7.328 \mathrm{E}+02 & 7.328 \mathrm{E}+02 & 7.328 \mathrm{E}+02 & 7.328 \mathrm{E}+02 & 7.328 \mathrm{E}+02 & 0.000 \mathrm{E}+00\end{array}$ $\begin{array}{llllll}7.328 \mathrm{E}+02 & 7.328 \mathrm{E}+02 & 7.328 \mathrm{E}+02 & 7.328 \mathrm{E}+02 & 7.328 \mathrm{E}+02 & 0.000 \mathrm{E}+00\end{array}$ $\begin{array}{llllll}7.328 \mathrm{E}+02 & 7.328 \mathrm{E}+02 & 7.328 \mathrm{E}+02 & 7.328 \mathrm{E}+02 & 7.328 \mathrm{E}+02 & 0.000 \mathrm{E}+00\end{array}$ $\begin{array}{llllll}1.107 \mathrm{E}+05 & 1.107 \mathrm{E}+05 & 1.943 \mathrm{E}+07 & 1.943 \mathrm{E}+07 & 1.943 \mathrm{E}+07 & 8.416 \mathrm{E}+00\end{array}$ $1.107 \mathrm{E}+05 \quad 1.107 \mathrm{E}+05 \quad 1.943 \mathrm{E}+07 \quad 1.943 \mathrm{E}+07 \quad 1.943 \mathrm{E}+07 \quad 8.416 \mathrm{E}+00$ $\begin{array}{lllllll}7.328 \mathrm{E}+02 & 7.328 \mathrm{E}+02 & 7.328 \mathrm{E}+02 & 7.328 \mathrm{E}+02 & 7.328 \mathrm{E}+02 & 0.000 \mathrm{E}+00\end{array}$ $\begin{array}{llllll}7.328 \mathrm{E}+02 & 7.328 \mathrm{E}+02 & 7.328 \mathrm{E}+02 & 7.328 \mathrm{E}+02 & 7.328 \mathrm{E}+02 & 0.000 \mathrm{E}+00\end{array}$ $\begin{array}{llllll}7.328 \mathrm{E}+02 & 7.328 \mathrm{E}+02 & 7.328 \mathrm{E}+02 & 7.328 \mathrm{E}+02 & 7.328 \mathrm{E}+02 & 0.000 \mathrm{E}+00\end{array}$ $\begin{array}{llllll}7.328 \mathrm{E}+02 & 7.328 \mathrm{E}+02 & 7.328 \mathrm{E}+02 & 7.328 \mathrm{E}+02 & 7.328 \mathrm{E}+02 & 0.000 \mathrm{E}+00\end{array}$ $\begin{array}{llllll}7.328 \mathrm{E}+02 & 7.328 \mathrm{E}+02 & 7.328 \mathrm{E}+02 & 7.328 \mathrm{E}+02 & 7.328 \mathrm{E}+02 & 0.000 \mathrm{E}+00\end{array}$ $\begin{array}{llllll}7.328 \mathrm{E}+02 & 7.328 \mathrm{E}+02 & 7.328 \mathrm{E}+02 & 7.328 \mathrm{E}+02 & 7.328 \mathrm{E}+02 & 0.000 \mathrm{E}+00\end{array}$ $\begin{array}{llllll}7.328 \mathrm{E}+02 & 7.328 \mathrm{E}+02 & 7.328 \mathrm{E}+02 & 7.328 \mathrm{E}+02 & 7.328 \mathrm{E}+02 & 0.000 \mathrm{E}+00\end{array}$ $\begin{array}{llllll}7.328 \mathrm{E}+02 & 7.328 \mathrm{E}+02 & 7.328 \mathrm{E}+02 & 7.328 \mathrm{E}+02 & 7.328 \mathrm{E}+02 & 0.000 \mathrm{E}+00\end{array}$ $\begin{array}{lllllll}7.342 \mathrm{E}+02 & 7.342 \mathrm{E}+02 & 4.460 \mathrm{E}+03 & 4.460 \mathrm{E}+03 & 4.460 \mathrm{E}+03 & 1.673 \mathrm{E}+00\end{array}$ $\begin{array}{llllll}7.339 \mathrm{E}+02 & 7.339 \mathrm{E}+02 & 7.339 \mathrm{E}+02 & 7.343 \mathrm{E}+02 & 7.343 \mathrm{E}+02 & 8.070 \mathrm{E}+01\end{array}$ $\begin{array}{llllll}7.498 \mathrm{E}+02 & 7.498 \mathrm{E}+02 & 7.498 \mathrm{E}+02 & 7.498 \mathrm{E}+02 & 7.498 \mathrm{E}+02 & 3.254 \mathrm{E}+00\end{array}$ $\begin{array}{lllll}7.931 \mathrm{E}+02 & 7.931 \mathrm{E}+02 & 7.931 \mathrm{E}+02 & 7.931 \mathrm{E}+02 & 7.931 \mathrm{E}+02 \quad 2.096 \mathrm{E}+01\end{array}$ $7.337 \mathrm{E}+02 \quad 7.337 \mathrm{E}+02 \quad 7.337 \mathrm{E}+02 \quad 7.342 \mathrm{E}+02 \quad 7.342 \mathrm{E}+02 \quad 1.201 \mathrm{E}+00$ $\begin{array}{llllll}7.328 \mathrm{E}+02 & 7.328 \mathrm{E}+02 & 7.328 \mathrm{E}+02 & 7.333 \mathrm{E}+02 & 7.333 \mathrm{E}+02 & 2.825 \mathrm{E}+01\end{array}$ $\begin{array}{llllll}7.905 \mathrm{E}+02 & 7.905 \mathrm{E}+02 & 7.905 \mathrm{E}+02 & 7.908 \mathrm{E}+02 & 7.908 \mathrm{E}+02 & 4.617 \mathrm{E}-01\end{array}$ $\begin{array}{lllllll}7.334 \mathrm{E}+02 & 7.334 \mathrm{E}+02 & 7.334 \mathrm{E}+02 & 7.334 \mathrm{E}+02 & 7.334 \mathrm{E}+02 & 1.525 \mathrm{E}+00\end{array}$ $\begin{array}{llllll}7.333 E+02 & 7.333 E+02 & 7.333 E+02 & 7.333 E+02 & 7.333 E+02 & 1.777 E+00\end{array}$ $\begin{array}{lllll}7.333 \mathrm{E}+02 & 7.333 \mathrm{E}+02 & 7.333 \mathrm{E}+02 & 7.333 \mathrm{E}+02 & 7.333 \mathrm{E}+02 \quad 1.690 \mathrm{E}+00\end{array}$ $\begin{array}{llllll}7.334 \mathrm{E}+02 & 7.334 \mathrm{E}+02 & 7.334 \mathrm{E}+02 & 7.334 \mathrm{E}+02 & 7.334 \mathrm{E}+02 & 1.525 \mathrm{E}+00\end{array}$ $\begin{array}{llllll}7.334 \mathrm{E}+02 & 7.334 \mathrm{E}+02 & 7.334 \mathrm{E}+02 & 7.334 \mathrm{E}+02 & 7.334 \mathrm{E}+02 & 1.525 \mathrm{E}+00\end{array}$ $\begin{array}{lllll}7.330 \mathrm{E}+02 & 7.330 \mathrm{E}+02 & 7.330 \mathrm{E}+02 & 7.330 \mathrm{E}+02 & 7.330 \mathrm{E}+02 \quad 2.566 \mathrm{E}+00\end{array}$ 
PropylB

Aldrin

Aroclor1016

Aroclor 1221

Aroclor 1232

DDD

DDE

DDT

Alpha-BHC

Beta-BHC

Delta-BHC

Acenaphthylene

Benzoic Acid

Dibenzofuran

Diethylphth

24-Dimethylphe

Dimethylphth

2Methylnaptha

2346Tetrachlor

Benzyl Alcohol

Acentonitrile

Acrolien

Acylonitrle

Bromodichloro

Bromoform

Bromometh

CarbonDis

Chlorometh

0-ChloroTu

Cumene

Dibromochloro

Dichlorodiflo

12cisDichloro

12 transDichl

12Dichlprop

Ethylbenz

Nhexane

Methyl Iso $\begin{array}{llllll}7.334 \mathrm{E}+02 & 7.334 \mathrm{E}+02 & 7.334 \mathrm{E}+02 & 7.334 \mathrm{E}+02 & 7.334 \mathrm{E}+02 & 1.525 \mathrm{E}+00\end{array}$

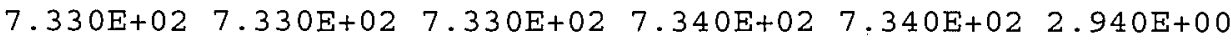

$8.347 \mathrm{E}+02 \quad 8.347 \mathrm{E}+02 \quad 8.347 \mathrm{E}+02 \quad 8.395 \mathrm{E}+02 \quad 8.372 \mathrm{E}+02 \quad 4.287 \mathrm{E}-01$

$\begin{array}{llllll}7.351 \mathrm{E}+02 & 7.351 \mathrm{E}+02 & 7.351 \mathrm{E}+02 & 7.354 \mathrm{E}+02 & 7.354 \mathrm{E}+02 & 7.641 \mathrm{E}-01\end{array}$

$\begin{array}{lllll}7.331 \mathrm{E}+02 & 7.331 \mathrm{E}+02 & 7.331 \mathrm{E}+02 & 7.332 \mathrm{E}+02 & 7.332 \mathrm{E}+02 \quad 2.273 \mathrm{E}+00\end{array}$

$8.493 \mathrm{E}+02 \quad 8.493 \mathrm{E}+02 \quad 8.493 \mathrm{E}+02 \quad 8.494 \mathrm{E}+02 \quad 8.494 \mathrm{E}+02 \quad 5.277 \mathrm{E}-01$

$8.270 \mathrm{E}+02 \quad 8.270 \mathrm{E}+02 \quad 8.270 \mathrm{E}+02 \quad 8.270 \mathrm{E}+02 \quad 8.270 \mathrm{E}+02 \quad 5.224 \mathrm{E}-01$

$1.149 \mathrm{E}+03 \quad 1.149 \mathrm{E}+03 \quad 1.149 \mathrm{E}+03 \quad 1.153 \mathrm{E}+03 \quad 1.152 \mathrm{E}+03 \quad 8.345 \mathrm{E}-01$

$7.342 \mathrm{E}+02 \quad 7.342 \mathrm{E}+02 \quad 7.342 \mathrm{E}+02 \quad 7.343 \mathrm{E}+02 \quad 7.343 \mathrm{E}+02 \quad 9.609 \mathrm{E}-01$

$\begin{array}{llllll}7.346 \mathrm{E}+02 & 7.346 \mathrm{E}+02 & 7.346 \mathrm{E}+02 & 7.346 \mathrm{E}+02 & 7.346 \mathrm{E}+02 & 8.399 \mathrm{E}-01\end{array}$

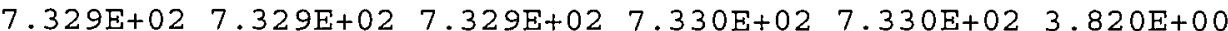

$7.337 \mathrm{E}+02 \quad 7.337 \mathrm{E}+02 \quad 7.337 \mathrm{E}+02 \quad 7.338 \mathrm{E}+02 \quad 7.338 \mathrm{E}+02 \quad 1.201 \mathrm{E}+00$

$\begin{array}{llllll}7.328 \mathrm{E}+02 & 7.328 \mathrm{E}+02 & 7.328 \mathrm{E}+02 & 7.328 \mathrm{E}+02 & 7.328 \mathrm{E}+02 & 1.266 \mathrm{E}+01\end{array}$

$7.352 \mathrm{E}+02 \quad 7.352 \mathrm{E}+02 \quad 7.352 \mathrm{E}+02 \quad 7.357 \mathrm{E}+02 \quad 7.357 \mathrm{E}+02 \quad 7.206 \mathrm{E}-01$

$7.328 \mathrm{E}+02 \quad 7.328 \mathrm{E}+02 \quad 7.328 \mathrm{E}+02 \quad 7.328 \mathrm{E}+02 \quad 7.328 \mathrm{E}+02 \quad 5.498 \mathrm{E}+00$

$\begin{array}{lllll}7.328 \mathrm{E}+02 & 7.328 \mathrm{E}+02 & 7.328 \mathrm{E}+02 & 7.329 \mathrm{E}+02 & 7.329 \mathrm{E}+02 \quad 7.604 \mathrm{E}+00\end{array}$

$\begin{array}{llllll}7.328 \mathrm{E}+02 & 7.328 \mathrm{E}+02 & 7.328 \mathrm{E}+02 & 7.328 \mathrm{E}+02 & 7.328 \mathrm{E}+02 & 1.898 \mathrm{E}+01\end{array}$

$7.342 \mathrm{E}+02 \quad 7.342 \mathrm{E}+02 \quad 7.342 \mathrm{E}+02 \quad 7.343 \mathrm{E}+02 \quad 7.343 \mathrm{E}+02 \quad 9.609 \mathrm{E}-01$

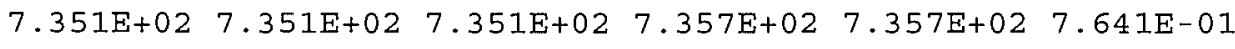

$7.328 \mathrm{E}+02 \quad 7.328 \mathrm{E}+02 \quad 7.328 \mathrm{E}+02 \quad 7.328 \mathrm{E}+02 \quad 7.328 \mathrm{E}+02 \quad 3.668 \mathrm{E}+01$

$\begin{array}{llllll}7.329 \mathrm{E}+02 & 7.329 \mathrm{E}+02 & 7.329 \mathrm{E}+02 & 7.329 \mathrm{E}+02 & 7.329 \mathrm{E}+02 & 2.529 \mathrm{E}+02\end{array}$

$\begin{array}{llllll}7.329 \mathrm{E}+02 & 7.329 \mathrm{E}+02 & 7.328 \mathrm{E}+02 & 7.329 \mathrm{E}+02 & 7.329 \mathrm{E}+02 & 1.813 \mathrm{E}+02\end{array}$

$\begin{array}{llllll}7.328 \mathrm{E}+02 & 7.328 \mathrm{E}+02 & 7.328 \mathrm{E}+02 & 7.328 \mathrm{E}+02 & 7.328 \mathrm{E}+02 & 1.138 \mathrm{E}+02\end{array}$

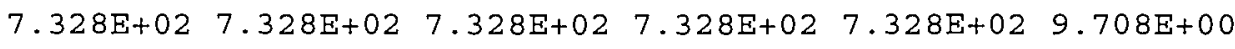

$7.328 \mathrm{E}+02 \quad 7.328 \mathrm{E}+02 \quad 7.328 \mathrm{E}+02 \quad 7.328 \mathrm{E}+02 \quad 7.328 \mathrm{E}+02 \quad 6.340 \mathrm{E}+00$

$\begin{array}{llllll}7.328 \mathrm{E}+02 & 7.328 \mathrm{E}+02 & 7.328 \mathrm{E}+02 & 7.328 \mathrm{E}+02 & 7.328 \mathrm{E}+02 & 3.246 \mathrm{E}+01\end{array}$

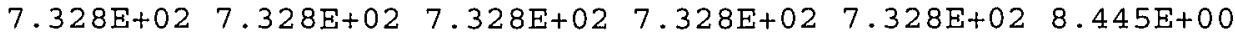

$\begin{array}{llllll}7.328 \mathrm{E}+02 & 7.328 \mathrm{E}+02 & 7.328 \mathrm{E}+02 & 7.328 \mathrm{E}+02 & 7.328 \mathrm{E}+02 & 4.637 \mathrm{E}+01\end{array}$

$\begin{array}{llllll}7.332 \mathrm{E}+02 & 7.333 \mathrm{E}+02 & 7.332 \mathrm{E}+02 & 7.333 \mathrm{E}+02 & 7.333 \mathrm{E}+02 & 1.775 \mathrm{E}+00\end{array}$

$\begin{array}{llllll}7.334 \mathrm{E}+02 & 7.334 \mathrm{E}+02 & 7.334 \mathrm{E}+02 & 7.335 \mathrm{E}+02 & 7.335 \mathrm{E}+02 & 1.525 \mathrm{E}+00\end{array}$

$\begin{array}{llllll}7.328 \mathrm{E}+02 & 7.328 \mathrm{E}+02 & 7.328 \mathrm{E}+02 & 7.328 \mathrm{E}+02 & 7.328 \mathrm{E}+02 & 8.445 \mathrm{E}+00\end{array}$

$\begin{array}{llllll}7.328 \mathrm{E}+02 & 7.328 \mathrm{E}+02 & 7.328 \mathrm{E}+02 & 7.328 \mathrm{E}+02 & 7.328 \mathrm{E}+02 & 8.445 \mathrm{E}+00\end{array}$

$7.328 \mathrm{E}+02 \quad 7.328 \mathrm{E}+02 \quad 7.328 \mathrm{E}+02 \quad 7.328 \mathrm{E}+02 \quad 7.328 \mathrm{E}+02 \quad 1.266 \mathrm{E}+01$

$\begin{array}{llllll}7.328 \mathrm{E}+02 & 7.328 \mathrm{E}+02 & 7.328 \mathrm{E}+02 & 7.328 \mathrm{E}+02 & 7.328 \mathrm{E}+02 & 8.431 \mathrm{E}+01\end{array}$

$7.328 \mathrm{E}+02 \quad 7.328 \mathrm{E}+02 \quad 7.328 \mathrm{E}+02 \quad 7.328 \mathrm{E}+02 \quad 7.328 \mathrm{E}+02 \quad 1.097 \mathrm{E}+01$

$7.330 \mathrm{E}+02 \quad 7.330 \mathrm{E}+02 \quad 7.330 \mathrm{E}+02 \quad 7.330 \mathrm{E}+02 \quad 7.330 \mathrm{E}+02 \quad 2.605 \mathrm{E}+00$

7. $342 \mathrm{E}+02 \quad 7.342 \mathrm{E}+02 \quad 7.342 \mathrm{E}+02 \quad 7.342 \mathrm{E}+02 \quad 7.342 \mathrm{E}+02 \quad 9.609 \mathrm{E}-01$

$\begin{array}{llllll}7.328 \mathrm{E}+02 & 7.328 \mathrm{E}+02 & 7.328 \mathrm{E}+02 & 7.328 \mathrm{E}+02 & 7.328 \mathrm{E}+02 & 3.246 \mathrm{E}+01\end{array}$ 
MethChoride PropGlycol

Styrene

1112Tetra

1122 Tetra

Trichloflo

123 TriChlopr

Trimethbenz

135 Trimeth

oxylene

acetophenone

Ethylchlorid

2 Hexanone

Methonal

Metacrylate

Chlorobenzene

Cyanide

Dibenz $[\mathrm{a}, \mathrm{h}]$

26Dinitrotoluene

DiNoctylphtalate

Endosulfan

$4 \mathrm{Ni}$ trobenzenamin

4 Nitrophenol

NNitrosodiphen

Xylene

12Dichloro

13Dichloro

14Dichloro

Methylcyclo

Benzo $[g, h, i]$

Methylchlor

2 MethylNap

2Nitrophenol

Aroclor 1268

EndosulfanII

Aldehyde

Ketone

Chlorobenzene $\begin{array}{llllll}7.328 \mathrm{E}+02 & 7.328 \mathrm{E}+02 & 7.328 \mathrm{E}+02 & 7.328 \mathrm{E}+02 & 7.328 \mathrm{E}+02 & 2.825 \mathrm{E}+01\end{array}$

$\begin{array}{llllll}7.334 \mathrm{E}+02 & 7.334 \mathrm{E}+02 & 7.332 \mathrm{E}+02 & 7.334 \mathrm{E}+02 & 7.334 \mathrm{E}+02 & 1.560 \mathrm{E}+03\end{array}$

$\begin{array}{llllll}7.329 \mathrm{E}+02 & 7.329 \mathrm{E}+02 & 7.329 \mathrm{E}+02 & 7.330 \mathrm{E}+02 & 7.330 \mathrm{E}+02 & 3.358 \mathrm{E}+00\end{array}$

$7.330 \mathrm{E}+02 \quad 7.330 \mathrm{E}+02 \quad 7.330 \mathrm{E}+02 \quad 7.330 \mathrm{E}+02 \quad 7.330 \mathrm{E}+02 \quad 2.940 \mathrm{E}+00$

$\begin{array}{llllll}7.328 \mathrm{E}+02 & 7.328 \mathrm{E}+02 & 7.328 \mathrm{E}+02 & 7.328 \mathrm{E}+02 & 7.328 \mathrm{E}+02 & 6.340 \mathrm{E}+00\end{array}$

$7.328 \mathrm{E}+02 \quad 7.328 \mathrm{E}+02 \quad 7.328 \mathrm{E}+02 \quad 7.328 \mathrm{E}+02 \quad 7.328 \mathrm{E}+02 \quad 5.498 \mathrm{E}+00$

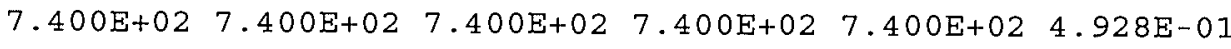

$\begin{array}{llllll}7.339 \mathrm{E}+02 & 7.339 \mathrm{E}+02 & 7.339 \mathrm{E}+02 & 7.339 \mathrm{E}+02 & 7.339 \mathrm{E}+02 & 1.077 \mathrm{E}+00\end{array}$

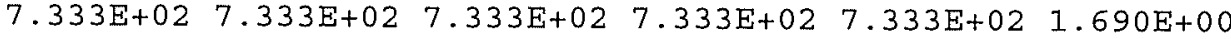

$\begin{array}{llllll}7.331 \mathrm{E}+02 & 7.331 \mathrm{E}+02 & 7.331 \mathrm{E}+02 & 7.331 \mathrm{E}+02 & 7.331 \mathrm{E}+02 & 2.273 \mathrm{E}+00\end{array}$

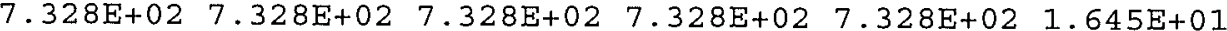

$\begin{array}{llllll}7.328 \mathrm{E}+02 & 7.328 \mathrm{E}+02 & 7.328 \mathrm{E}+02 & 7.328 \mathrm{E}+02 & 7.328 \mathrm{E}+02 & 2.488 \mathrm{E}+01\end{array}$

$\begin{array}{llllll}7.328 \mathrm{E}+02 & 7.328 \mathrm{E}+02 & 7.328 \mathrm{E}+02 & 7.328 \mathrm{E}+02 & 7.328 \mathrm{E}+02 & 2.488 \mathrm{E}+01\end{array}$

$\begin{array}{llllll}7.330 \mathrm{E}+02 & 7.330 \mathrm{E}+02 & 7.329 \mathrm{E}+02 & 7.330 \mathrm{E}+02 & 7.330 \mathrm{E}+02 & 4.637 \mathrm{E}+02\end{array}$

$\begin{array}{llllll}7.328 \mathrm{E}+02 & 7.328 \mathrm{E}+02 & 7.328 \mathrm{E}+02 & 7.328 \mathrm{E}+02 & 7.328 \mathrm{E}+02 & 2.825 \mathrm{E}+01\end{array}$

$\begin{array}{llllll}7.329 \mathrm{E}+02 & 7.329 \mathrm{E}+02 & 9.468 \mathrm{E}+02 & 9.468 \mathrm{E}+02 & 9.468 \mathrm{E}+02 & 3.820 \mathrm{E}+00\end{array}$

$\begin{array}{lllllll}7.328 \mathrm{E}+02 & 7.328 \mathrm{E}+02 & 7.570 \mathrm{E}+02 & 7.582 \mathrm{E}+02 & 7.582 \mathrm{E}+02 & 3.668 \mathrm{E}+01\end{array}$

$1.898 \mathrm{E}+03 \quad 1.898 \mathrm{E}+03 \quad 1.941 \mathrm{E}+03 \quad 1.955 \mathrm{E}+03 \quad 1.949 \mathrm{E}+03 \quad 1.255 \mathrm{E}+00$

$\begin{array}{llllll}7.328 \mathrm{E}+02 & 7.328 \mathrm{E}+02 & 7.756 \mathrm{E}+02 & 7.756 \mathrm{E}+02 & 7.756 \mathrm{E}+02 & 1.645 \mathrm{E}+01\end{array}$

$\begin{array}{llllll}2.962 E+05 & 2.962 E+05 \quad 2.961 E+05 & 2.968 E+05 & 2.962 E+05 & 1.308 E+01\end{array}$

$7.334 \mathrm{E}+02 \quad 7.334 \mathrm{E}+02 \quad 3.661 \mathrm{E}+04 \quad 3.661 \mathrm{E}+04 \quad 3.661 \mathrm{E}+04 \quad 1.444 \mathrm{E}+00$

$\begin{array}{llllll}7.328 \mathrm{E}+02 & 7.328 \mathrm{E}+02 & 7.357 \mathrm{E}+03 & 7.357 \mathrm{E}+03 & 7.357 \mathrm{E}+03 & 2.867 \mathrm{E}+01\end{array}$

$\begin{array}{lllllll}7.328 \mathrm{E}+02 & 7.328 \mathrm{E}+02 & 2.872 \mathrm{E}+03 & 2.872 \mathrm{E}+03 & 2.872 \mathrm{E}+03 & 1.266 \mathrm{E}+01\end{array}$

$\begin{array}{llllll}7.330 \mathrm{E}+02 & 7.330 \mathrm{E}+02 & 7.696 \mathrm{E}+02 & 7.696 \mathrm{E}+02 & 7.696 \mathrm{E}+02 & 2.605 \mathrm{E}+00\end{array}$

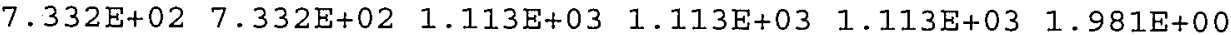

$\begin{array}{llllll}7.332 \mathrm{E}+02 & 7.333 \mathrm{E}+02 & 1.334 \mathrm{E}+03 & 1.334 \mathrm{E}+03 & 1.334 \mathrm{E}+03 & 1.775 \mathrm{E}+00\end{array}$

$\begin{array}{lllllll}7.335 \mathrm{E}+02 & 7.335 \mathrm{E}+02 & 1.424 \mathrm{E}+03 & 1.424 \mathrm{E}+03 & 1.424 \mathrm{E}+03 & 1.362 \mathrm{E}+00\end{array}$

$\begin{array}{llllll}7.332 \mathrm{E}+02 & 7.332 \mathrm{E}+02 & 1.347 \mathrm{E}+03 & 1.347 \mathrm{E}+03 & 1.347 \mathrm{E}+03 & 1.775 \mathrm{E}+00\end{array}$

$\begin{array}{llllll}7.329 \mathrm{E}+02 & 7.329 \mathrm{E}+02 & 1.561 \mathrm{E}+03 & 1.561 \mathrm{E}+03 & 1.561 \mathrm{E}+03 & 3.526 \mathrm{E}+00\end{array}$

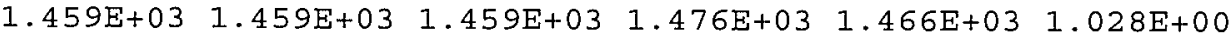

$\begin{array}{lllllll}7.328 \mathrm{E}+02 & 7.328 \mathrm{E}+02 & 7.328 \mathrm{E}+02 & 7.328 \mathrm{E}+02 & 7.328 \mathrm{E}+02 & 4.637 \mathrm{E}+01\end{array}$

$7.342 \mathrm{E}+02 \quad 7.342 \mathrm{E}+02 \quad 7.342 \mathrm{E}+02 \quad 7.343 \mathrm{E}+02 \quad 7.343 \mathrm{E}+02 \quad 9.609 \mathrm{E}-01$

$\begin{array}{llllll}7.328 \mathrm{E}+02 & 7.328 \mathrm{E}+02 & 7.328 \mathrm{E}+02 & 7.328 \mathrm{E}+02 & 7.328 \mathrm{E}+02 & 1.519 \mathrm{E}+01\end{array}$

$3.005 \mathrm{E}+03 \quad 3.005 \mathrm{E}+03 \quad 3.005 \mathrm{E}+03 \quad 3.097 \mathrm{E}+03 \quad 3.020 \mathrm{E}+03 \quad 1.638 \mathrm{E}+00$

$\begin{array}{lllllll}7.334 \mathrm{E}+02 & 7.334 \mathrm{E}+02 & 7.334 \mathrm{E}+02 & 7.334 \mathrm{E}+02 & 7.334 \mathrm{E}+02 & 1.444 \mathrm{E}+00\end{array}$

$7.400 \mathrm{E}+02 \quad 7.400 \mathrm{E}+02 \quad 7.400 \mathrm{E}+02 \quad 7.401 \mathrm{E}+02 \quad 7.401 \mathrm{E}+02 \quad 4.928 \mathrm{E}-01$

$7.400 \mathrm{E}+02 \quad 7.400 \mathrm{E}+02 \quad 7.400 \mathrm{E}+02 \quad 7.401 \mathrm{E}+02 \quad 7.401 \mathrm{E}+02 \quad 4.928 \mathrm{E}-01$

$\begin{array}{lllll}7.329 \mathrm{E}+02 & 7.329 \mathrm{E}+02 & 7.329 \mathrm{E}+02 & 7.329 \mathrm{E}+02 & 7.329 \mathrm{E}+02 \quad 3.820 \mathrm{E}+00\end{array}$ 
Vinyl Chloride

o-cresol

m-cresol

$\mathrm{p}$-cresol

14Dichlorobenzen

Hexachlorobenzen

Hexachlorobutadn

Hexachloroethane

Nitrobenzene

246-Trichlorphn]

Pyridine

24-D

245-TP (Silvex)

Chlordane

Endrin

Heptachlor

Heptachlor epoxd

Lindane

Methoxychlor

Toxaphene

Lithium

124 trimethylb

1hexanol

2 hexanone

butylbenzene $\begin{array}{llllll}7.328 \mathrm{E}+02 & 7.328 \mathrm{E}+02 & 7.328 \mathrm{E}+02 & 7.328 \mathrm{E}+02 & 7.328 \mathrm{E}+02 & 2.488 \mathrm{E}+01\end{array}$

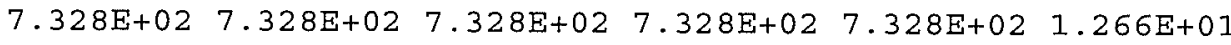
$\begin{array}{llllll}7.328 \mathrm{E}+02 & 7.328 \mathrm{E}+02 & 7.328 \mathrm{E}+02 & 7.328 \mathrm{E}+02 & 7.328 \mathrm{E}+02 & 1.097 \mathrm{E}+01\end{array}$ $\begin{array}{llllll}7.328 \mathrm{E}+02 & 7.328 \mathrm{E}+02 & 7.328 \mathrm{E}+02 & 7.328 \mathrm{E}+02 & 7.328 \mathrm{E}+02 & 1.266 \mathrm{E}+01\end{array}$ $\begin{array}{llllll}7.332 \mathrm{E}+02 & 7.332 \mathrm{E}+02 & 7.332 \mathrm{E}+02 & 7.333 \mathrm{E}+02 & 7.333 \mathrm{E}+02 & 1.775 \mathrm{E}+00\end{array}$ $\begin{array}{llllll}7.695 \mathrm{E}+02 & 7.695 \mathrm{E}+02 & 7.695 \mathrm{E}+02 & 7.696 \mathrm{E}+02 & 7.696 \mathrm{E}+02 & 4.244 \mathrm{E}-01\end{array}$ $\begin{array}{llllll}7.444 \mathrm{E}+02 & 7.444 \mathrm{E}+02 & 7.444 \mathrm{E}+02 & 7.444 \mathrm{E}+02 & 7.444 \mathrm{E}+02 & 4.397 \mathrm{E}-01\end{array}$ $7.342 \mathrm{E}+02 \quad 7.342 \mathrm{E}+02 \quad 7.342 \mathrm{E}+02 \quad 7.343 \mathrm{E}+02 \quad 7.343 \mathrm{E}+02 \quad 9.609 \mathrm{E}-01$ $\begin{array}{llllll}7.328 \mathrm{E}+02 & 7.328 \mathrm{E}+02 & 7.328 \mathrm{E}+02 & 7.328 \mathrm{E}+02 & 7.328 \mathrm{E}+02 & 1.434 \mathrm{E}+01\end{array}$ $\begin{array}{llllll}7.337 \mathrm{E}+02 & 7.337 \mathrm{E}+02 & 7.337 \mathrm{E}+02 & 7.337 \mathrm{E}+02 & 7.337 \mathrm{E}+02 & 1.201 \mathrm{E}+00\end{array}$ $7.328 \mathrm{E}+02 \quad 7.328 \mathrm{E}+02 \quad 7.328 \mathrm{E}+02 \quad 7.328 \mathrm{E}+02 \quad 7.328 \mathrm{E}+02 \quad 2.825 \mathrm{E}+01$

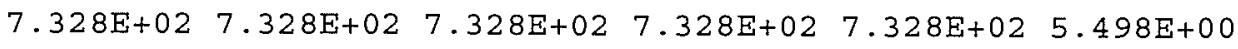
$\begin{array}{llllll}7.342 \mathrm{E}+02 & 7.342 \mathrm{E}+02 & 7.342 \mathrm{E}+02 & 7.342 \mathrm{E}+02 & 7.342 \mathrm{E}+02 & 9.609 \mathrm{E}-01\end{array}$ $7.905 \mathrm{E}+02 \quad 7.905 \mathrm{E}+02 \quad 7.905 \mathrm{E}+02 \quad 7.907 \mathrm{E}+02 \quad 7.907 \mathrm{E}+02 \quad 4.617 \mathrm{E}-01$

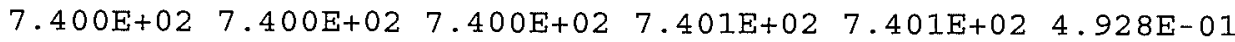
$\begin{array}{llllll}7.365 \mathrm{E}+02 & 7.365 \mathrm{E}+02 & 7.365 \mathrm{E}+02 & 7.366 \mathrm{E}+02 & 7.366 \mathrm{E}+02 & 6.144 \mathrm{E}-01\end{array}$ $\begin{array}{llllll}7.789 \mathrm{E}+02 & 7.789 \mathrm{E}+02 & 7.789 \mathrm{E}+02 & 7.789 \mathrm{E}+02 & 7.789 \mathrm{E}+02 \quad 4.365 \mathrm{E}-01\end{array}$

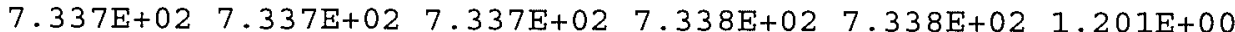
$7.374 \mathrm{E}+02 \quad 7.374 \mathrm{E}+02 \quad 7.374 \mathrm{E}+02 \quad 7.377 \mathrm{E}+02 \quad 7.377 \mathrm{E}+02 \quad 5.888 \mathrm{E}-01$

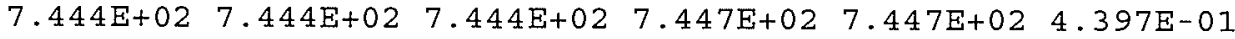
$\begin{array}{llllll}9.237 \mathrm{E}+02 & 9.237 \mathrm{E}+02 & 9.237 \mathrm{E}+02 & 9.249 \mathrm{E}+02 & 9.249 \mathrm{E}+02 & 1.200 \mathrm{E}+00\end{array}$ $\begin{array}{llllll}7.339 \mathrm{E}+02 & 7.339 \mathrm{E}+02 & 7.339 \mathrm{E}+02 & 7.339 \mathrm{E}+02 & 7.339 \mathrm{E}+02 & 1.077 \mathrm{E}+00\end{array}$ $\begin{array}{llllll}7.328 \mathrm{E}+02 & 7.328 \mathrm{E}+02 & 7.328 \mathrm{E}+02 & 7.328 \mathrm{E}+02 & 7.328 \mathrm{E}+02 & 2.488 \mathrm{E}+01\end{array}$ $\begin{array}{llllll}7.328 \mathrm{E}+02 & 7.328 \mathrm{E}+02 & 7.328 \mathrm{E}+02 & 7.328 \mathrm{E}+02 & 7.328 \mathrm{E}+02 & 2.488 \mathrm{E}+01\end{array}$ $\begin{array}{llllll}7.334 \mathrm{E}+02 & 7.334 \mathrm{E}+02 & 7.334 \mathrm{E}+02 & 7.334 \mathrm{E}+02 & 7.334 \mathrm{E}+02 & 1.525 \mathrm{E}+00\end{array}$ 
$* * * * * * * * * *$ Image of Input Files $* * * * * * * * * *$

-- Input File: ABCDEF.DAT

W. A. C. - August, 2009 New Proposed Cell 1-5 EMWMF $202,0,5$

1,2

$0 ., 166 ., 525 ., 2.23 \mathrm{E}+5,1 ., 73.9,0$.

$1800,6,0 ., 0,0,0 ., 315,0$.

$20,2,0,1,1$

$4.0,15.3,1324061 \ldots,-1 ., 0 ., 1600 ., 40, .705,0.90,1$.

$1.0 \mathrm{E}-7,8000 \ldots .705,0 ., 1.0 \mathrm{E}+0,0.01,0 \mathrm{E}+0$

$240 ., 5.56 \mathrm{E}-4, .22, .02,3.0 \mathrm{E}-4,20 ., .01$

$4,6.3, .23,0 ., 1.1 \mathrm{E}-06, .01,0 ., 0 ., 0 ., 0 ., 0$

$0,0,0,0,0,0,0$

$1,0,0,1$

$0.0091,4.6,0.04,4.6, .025,10 ., 0.00001,1,0 ., 25$

- Input File: BRCDCF.DAT

501, Acenaphthene

502 , Acetone

503, Anthracene

504, Aluminum

505, Antimony

506, Arochlors-1242

507, Arochlors -1254

508, Arochlors -1248

509, Arochlors -1260

510, Arsenic

511, Barium

512 , Benzene

513, BenzoAanthracene

514, Beryllium

515, Bis2ethylhexapht

516, Butylbenzylphtha

517, Cadmium

518, Calcium

$\begin{array}{rrrr}0 ., & 6.0 \mathrm{E}-01, & 0 ., & 0 . \\ 0 ., & 1.0 \mathrm{E}-01, & 0 ., & 0 . \\ 0 ., & 3.0 \mathrm{E}-01, & 0 ., & 0 . \\ 0 ., 4.0 \mathrm{E}-04, & 0 ., & 0 . \\ 0 ., 4.0 \mathrm{E}-04, & 0 ., & 0 . \\ 7.7, & 0 ., & 0 ., & 0 . \\ 0 ., 2.0 \mathrm{E}-05, & 0 ., & 0 . \\ 7.7, & 0 ., & 0 ., & 0 . \\ 7.7, & 0 ., & 0 ., & 0 . \\ 1.5 \mathrm{E}+00,3.0 \mathrm{E}-04, & 0 ., & 0 . \\ 0 ., 7.0 \mathrm{E}-02, & 0 ., & 0 . \\ 2.9 \mathrm{E}-02, & 0 ., & 0 ., & 0 . \\ 7.3 \mathrm{E}-01, & 0 ., & 0 ., & 0 . \\ 4.3 \mathrm{E}+00, & 5.0 \mathrm{E}-03, & 0 ., & 0 . \\ 1.4 \mathrm{E}-02,2.0 \mathrm{E}-02, & 0 ., & 0 . \\ 0 ., 2.0 \mathrm{E}-01, & 0 ., & 0 . \\ 0 ., & 5.0 \mathrm{E}-04, & 0 ., & 0 . \\ 0 ., & 0 ., & 0 ., & 0 .\end{array}$


519, Carbazole

520, Carbontetchl

521, Chloroform

522, Chromium III

523, Chromium VI

524 , Cobalt

525 , Copper

526 , Benzobfl ranthene

527 , BenzoKflranthene

528 , Benzoghiperylene

529, Benzo (a) pyrene

530, Dibenzoahanthrac

531, Dibenzofuran

532, Dieldrin

533, Dinbutylphthalat

534, Dinoctylphthalat

535,24-dinitrotoluen

536, Fluoranthene

537, Flourene

538, 2-Hexanone

539, Indeno123cdpyren

540 , Iron

541 , Isophorone

542 , Lead

543, Lithium

544, Magnesium

545, Manganese

546, Mercury

547, Methchloride

548,2 Methylnaphthale

549, Naphthalene

550, Nickel

551, NnitroNpropyl

552, Pentachloropheno

553, Phenanthrene

554, Phenol

555, Potassium

556, Pyrene

\begin{tabular}{|c|c|c|c|}
\hline $2.0 \mathrm{E}-02$ & 0. & 0. & 0 \\
\hline 1.3E-01, & $7.0 e-04$ & 0. & 0. \\
\hline $6.1 E-03$ & 1.0e-02, & 0. & 0. \\
\hline 0. & $1.0 e+00$ & 0. & 0 \\
\hline $0 .$, & 5. $0 \mathrm{E}-03$ & 0. & 0 \\
\hline 0. & $6.0 e-02$ & 0. & 0 \\
\hline 0. & $3.7 e-02$ & 0. & 0 \\
\hline 7.3E-01, & 0. & 0. & 0 \\
\hline $7.3 E-02$, & 0. & 0. & 0. \\
\hline 0. & 0. & 0. & 0. \\
\hline 7. $3 \mathrm{E}+00$, & 0. & 0. & 0 \\
\hline 7. $3 E+00$ & 0. & 0. & 0 \\
\hline 0. & $4.0 e-03$ & 0. & 0 \\
\hline 1.3E+01, & $5.0 e-05$ & 0. & 0 \\
\hline 0. & 1.0e-01, & 0. & 0. \\
\hline 0. & 0. & 0. & 0 \\
\hline $6.8 E-04$ & 2.0E-03, & 0. & 0 \\
\hline 0. & 4. $0 \mathrm{E}-02$ & 0. & 0 \\
\hline 0. & $4.0 E-02$ & 0. & 0 \\
\hline 0. & 0. & 0. & 0 \\
\hline $7.3 \mathrm{E}-01$ & 0. & 0. & 0 \\
\hline 0. & $8.6 \mathrm{E}-03$ & 0. & 0 \\
\hline $9.5 E-04$ & $2.0 \mathrm{e}-01$ & 0. & 0 \\
\hline $0 .$, & 1. $4 \mathrm{E}-03$, & 0. & 0 \\
\hline $0 .$, & $2.0 e-02$ & 0. & 0 \\
\hline $0 .$, & 0. & 0. & 0 \\
\hline 0. & 4. $7 \mathrm{E}-02$ & 0. & 0. \\
\hline 0 & $3.0 E-04$ & 0. & 0 \\
\hline 7. 5E-03, & $6.0 e-02$ & 0. & 0 . \\
\hline 1. $43 e-2$ & $6.0 e-02$ & 0. & 0 \\
\hline $0 .$, & $3.6 \mathrm{E}-02$ & 0. & 0 \\
\hline $0 .$, & $2.0 e-02$ & 0. & 0 \\
\hline $7.0 \mathrm{E}+00$ & 0. & 0. & 0. \\
\hline 1.2E-01, & $3.0 e-02$ & 0. & 0 \\
\hline 1. $15 \mathrm{E}+1$ & 0. & 0. & 0 \\
\hline 0. & 6.0E-01, & 0. & 0 \\
\hline 0. & 0. & 0. & 0 \\
\hline 0. & $3.0 \mathrm{E}-02$ & 0. & 0 \\
\hline
\end{tabular}




\begin{tabular}{|c|c|c|c|c|}
\hline 557, Selenium & 0. & $5.0 \mathrm{E}-03$ & 0. & 0 . \\
\hline 558, Silver & $0 .$, & $5.0 E-03$ & 0. & 0 . \\
\hline 559, Sodium & $0 .$, & 0. & $0 .$, & 0 . \\
\hline 560 , strontium & $0 .$, & $6.0 e-01$ & 0. & 0 . \\
\hline 561 , Tetrachloroethen & $5.2 \mathrm{E}-02$ & $1.0 e-02$ & $0 . r$ & 0 . \\
\hline 562, Thallium & 0. & $8.0 e-05$ & $0 .$, & 0 . \\
\hline 563 , Tin & 0. & 6.0E-01, & $0 .$, & 0 . \\
\hline 564, Toluene & 0. & $2.0 e-01$ & 0. & 0 . \\
\hline 565, Trichloroethene & 1.1E-02, & 0. & $0 .$, & 0. \\
\hline $566, U-232$ & 0. & $3.0 \mathrm{E}-03$ & 0. & 0 . \\
\hline $567, \mathrm{U}-233$ & 0. & $3.0 E-03$ & $0 .$, & 0 . \\
\hline $568, \mathrm{U}-234$ & 0. & $3.0 E-03$ & $0 .$, & 0 . \\
\hline $569, U-235$ & 0. & $3.0 E-03$ & $0 .$, & 0 . \\
\hline $570, U-236$ & 0. & $3.0 \mathrm{E}-03$ & 0. & 0 . \\
\hline $571, U-238$ & 0. & 3.0E-03, & 0. & 0 . \\
\hline 572, Vanadium & 0. & 7.0E-03, & 0. & 0 . \\
\hline 573, Zinc & 0. & 3. $0 \mathrm{E}-01$ & 0. & 0. \\
\hline 574, Zirconium & 0. & $3.5 e-02$ & 0. & 0. \\
\hline $575,12378 \mathrm{PeCDF}$ & $7.5 e+04$ & $0 .$, & 0. & 0. \\
\hline $576,23478 \mathrm{PeCDF}$ & $7.5 e+03$ & 0. & 0. & 0. \\
\hline $577, \mathrm{OCDD}$ & $1.5 e+02$, & 0. & 0. & 0 . \\
\hline $578, \mathrm{OCDF}$ & $1.5 e+02$, & 0. & 0. & 0 . \\
\hline $579,1234678 \mathrm{HpCDD}$ & 1. $5 e+03$ & 0. & 0. & 0 . \\
\hline $580,1234678 \mathrm{HpCDF}$ & 1. $5 e+03$ & 0. & 0. & 0. \\
\hline $581,123478 \mathrm{HxCDF}$ & $1.5 e+04$ & 0. & 0. & 0 . \\
\hline $582,12378 \mathrm{HxCDD}$ & $1.5 e+04$ & 0. & 0. & 0. \\
\hline $583,123678 \mathrm{H} \times \mathrm{CDD}$ & $1.5 e+04$ & 0. & 0. & 0 . \\
\hline $584,123789 \mathrm{H} \times \mathrm{CDD}$ & $1.5 e+04$ & 0. & 0. & 0 . \\
\hline $585,2378 \mathrm{TCDD}$ & $1.5 e+04$ & 0. & $0 .$, & 0 . \\
\hline $586,2378 \mathrm{TCDF}$ & $1.5 e+04$ & 0. & $0 .$, & 0 . \\
\hline 590, Hexachloroethene & 1. $4 e-02$, & $1.0 e-03$ & 0. & 0 . \\
\hline 591, Trichlorobenzene & 0. & $1.0 e-02$ & 0. & 0 . \\
\hline 592,124 Trichlorb & $0.00 e+00$ & $5.0 e-02$ & 0. & 0 . \\
\hline 593, Molybdenum & $0.00 e+0$ & $5.0 \mathrm{E}-03$ & 0. & 0 . \\
\hline 594, strontium & $0.00 \mathrm{E}+0$ & $6.0 e-01$ & 0. & 0. \\
\hline 595, Acenaphthylene & $0.00 e+0$ & $0.00 e+0$ & 0. & 0 . \\
\hline 596, benzidine & $2.3 \mathrm{E}+02$ & $3.0 e-03$ & 0. & 0 . \\
\hline 597, Chlordane & $3.5 \mathrm{E}-01$ & 5. $0 E-04$ & 0. & 0 . \\
\hline
\end{tabular}


619, MethylPropylB 620,124 TrimethylB 621, 135TrimethylB 622, MethylEthylB 623, ButylB

624,12 DiMethylB

625 , PropylB

626, Aldrin

627, Aroclor 1016

628, Aroclor 1221

629, Aroclor 1232

$630, \mathrm{DDD}$

$631, \mathrm{DDE}$

$632, \mathrm{DDT}$

633, Alpha-BHC

634, Beta-BHC

635, Delta-BHC

636, Acenaphthylene

637, Benzoic Acid

638, Dibenzofuran

639, Diethylphth

640,24-Dimethylphe

641, Dimethylphth

642,2 Methylnaptha

643,2346 Tetrachlor

644, Benzyl Alcohol

645, Acentonitrile

646, Acrolien

647, Acylonitrle

648, Bromodichloro

649, Bromoform

650, Bromometh

651, Carbondis

652, Chlorometh

653, 0-ChloroTu

654, cumene

655, Dibromochloro

656, Dichlorodiflo

$\begin{array}{lll}0 ., 3.7 e-02, & 0 ., & 0 . \\ 0 ., 5.0 e-02, & 0 ., & 0 . \\ 0 ., 5.0 e-02, & 0 ., & 0 . \\ 0 ., 3.7 e-02, & 0 ., & 0 . \\ 0 ., 3.7 \mathrm{E}-02, & 0 ., & 0 . \\ 0 ., 2.0 \mathrm{e}+00, & 0 ., & 0 . \\ 0 ., 3.7 \mathrm{e}-02, & 0 ., & 0 .\end{array}$

1.7E+01, 3.0e-05

4.0E-01, 7.0e-05,

4. OE-01, 0 .,

4. $0 \mathrm{E}-01$,

0 .

2. $4 \mathrm{E}-01$,

0.

$3.4 E-01,0$.

3. $4 \mathrm{E}-01,5.0 \mathrm{E}-04$,

6. $3 \mathrm{E}+00, \quad 0$.,

1. $8 \mathrm{E}+00$, 0 .,

1.8E+00, 0.

$0 ., 6.0 e-02$

$0 ., 4.0 e+00$,

$0 ., 4.0 e-03$,

$0 ., 8.0 e-01$,

$0 ., 2.0 e-02$,

$0 ., 1.0 \mathrm{E}+01$,

$0.4 .0 e-03$

$0 ., 3.0 e-02$,

$0 ., 3.0 e-01$

$0 ., 6.0 e-03$

$0 ., 5.0 e-04$

5. 4E-01, 1.OE-03,

$6.2 \mathrm{E}-02,2 . \mathrm{OE}-02$

7.9E-03, 2. OE-02, $0 ., 1.4 \mathrm{E}-03$

$0 ., 1.0 \mathrm{E}-01$,

1. 3E-02, 0 . ,

$0 ., 2.0 \mathrm{E}-02$,

0., 1.0E-01,

8.4E-02, 2.0E-02,

$0 ., 2.0 e-01$,
0 .

0 ,

0.

0.

$0 .$,

0 .,

0 .,

0 .,

0.

0 . ,

$0 ., \quad 0$.

$0 ., \quad 0$

$0 ., \quad 0$.

$0 ., \quad 0$.

$0 ., \quad 0$

$0 ., \quad 0$.

$0 ., \quad 0$

$0 ., \quad 0$.

$0 ., \quad 0$.

$0 ., \quad 0$.

$0 ., \quad 0$

$0 ., \quad 0$.

o., 0 .

o., 0 .

o., 0 .

o., 0 .

o., 0 .

$0 ., \quad 0$.

$0 ., \quad 0$

$0 ., 0$

0 .

0.

0 .

$$
0 \text {. }
$$$$
0 .
$$

.$$
\text { . }
$$

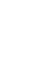

.

.

$$
\text { . }
$$




\begin{tabular}{|c|c|c|c|}
\hline $657,12 \mathrm{cisDichloro}$ & $0 ., 1.0 e-02$, & 0. & 0 \\
\hline 658,12 transDichl & $0 ., 2.0 \mathrm{E}-02$, & 0. & 0 \\
\hline 659,12 Dichlprop & 6.8E-02, $\quad 0 .$, & 0. & 0. \\
\hline 660, Ethylbenz & $0.1 .0 E-01$, & 0. & 0 \\
\hline 661, Nhexane & $0 ., 6.0 e-02$, & 0. & 0 \\
\hline 662 , MethylIso & $0 ., 8.0 e-02$, & 0. & 0 . \\
\hline 664, PropGlycol & $0 ., 5.0 e-01$, & 0. & 0 . \\
\hline 665, styrene & $0 ., 2.0 e-01$ & 0. & 0 \\
\hline 666,1112 Tetra & $2.6 \mathrm{E}-02,3.0 \mathrm{e}-02$ & 0. & 0 \\
\hline 667,1122 Tetra & $2.0 \mathrm{E}-01,6.0 \mathrm{e}-02$, & 0. & 0 \\
\hline 668,Trichloflo & $0 ., 3.0 \mathrm{E}-01$ & 0. & 0 \\
\hline 669, 123Trichlopr & $7.0 \mathrm{E}+00,6.0 \mathrm{e}-03$ & 0. & 0 \\
\hline 670, Trimethbenz & $0 ., 5.0 \mathrm{e}-02$, & 0. & 0 \\
\hline 671,135 Trimeth & $0 ., 5.0 \mathrm{e}-02$, & 0. & 0 \\
\hline 672 , oxylene & $0 ., 2.0 e+00$ & $0 .$, & 0 \\
\hline 673 , acetophenone & $0 ., 1.0 \mathrm{e}-01$, & 0. & 0 \\
\hline 674 , Ethylchlorid & $2.9 \mathrm{E}-03,4.0 \mathrm{E}-01$, & 0. & 0 \\
\hline 675,2 Hexanone & $0.4 .0 e-02$, & 0. & 0 \\
\hline 676 , Methonal & $0.5 .0 e-01$ & 0. & 0 \\
\hline 677, MMetacrylate & $0 ., 1.40 e+00$ & 0. & 0 \\
\hline 678 , Chlorobenzene & $0 ., 2.0 e-02$, & 0. & 0 \\
\hline 679, Cyanide & $0.2 .0 e-02$, & 0. & 0 \\
\hline 680, Dibenz $[a, h]$ & $7.3 e+00$ & 0. & 0 \\
\hline 682,26 Dinitrotoluene & $6.8 e-01,1.0 e-03$, & 0. & 0. \\
\hline 683, DiNOctylphtalate & $0 ., 4.0 e-02$, & 0. & 0. \\
\hline 684, Endosulfan & $0 ., 6.0 e-03$ & 0. & 0. \\
\hline $685,4 \mathrm{Ni}$ trobenzenamin & $2.1 \mathrm{E}-02,3.0 \mathrm{e}-03$ & 0. & 0. \\
\hline $686,4 \mathrm{Nitrophenol}$ & $0.6 .2 e-02$, & 0. & 0. \\
\hline 687, NNitrosodiphen & $4.9 e-03,2.0 e-02$, & 0 & 0 \\
\hline 688, xylene & $0 ., 2.0 e-01$ & 0. & 0. \\
\hline $689,12 \mathrm{Dichloro}$ & $0 ., 9.0 \mathrm{e}-02$, & 0. & 0. \\
\hline 690,13 Dichloro & $0 ., 8.9 e-02$, & 0. & 0 \\
\hline 691, 14Dichloro & $2.4 e-02,2.3 e-01$, & 0. & 0 \\
\hline 692, Methylcyclo & $0 ., 6.0 e-02$ & 0. & 0 \\
\hline $693, \operatorname{Benzo}[\mathrm{g}, \mathrm{h}, \mathrm{i}]$ & $7.30 \mathrm{E}+00$, & 0. & 0 \\
\hline 694, MethylChlor & 1.3E-02, & 0. & 0 \\
\hline 695,2 Methylnap & $0 ., 4.00 \mathrm{E}-03$ & 0. & 0 \\
\hline 696,2 Nitrophenol & $0 ., 6.20 \mathrm{E}-02$, & 0. & 0 \\
\hline
\end{tabular}


697, Aroclor 1268

698, EndosulfanII

699, Aldehyde

700 , Ketone

701, Chlorobenzene

702, Vinyl Chloride

703,o-cresol

$704, \mathrm{~m}$-cresol

705,p-cresol

706, 14Dichlorobenzen

707, Hexachlorobenzen

709, Hexachloroethane

710, Nitrobenzene

711,246-Trichlorphnl

712, Pyridine

$713,24-\mathrm{D}$

$714,245-T P$ (Silvex)

715, Chlordane

716, Endrin

717, Heptachlor

718 , Heptachlor epoxd

719, Lindane

720 , Methoxychlor

721, Toxaphene

722 , Lithium

723,124 trimethylb

724 , 1hexanol

725,2 hexanone

726 , butylbenzene
708, Hexachlorobutadn

\begin{tabular}{|c|c|c|}
\hline $7.7 \mathrm{E}+00$ & $0 .$, & 0. \\
\hline 0. & $6.0 e-03$ & 0. \\
\hline 0. & $3.0 E-04$ & 0. \\
\hline 0. & 3.0e-04, & 0. \\
\hline 0. & $2.0 e-02$, & 0. \\
\hline $1.4 \mathrm{e}+00$ & $3.0 e-03$ & 0. \\
\hline 0 & $5.0 e-02$, & 0. \\
\hline 0. & $5.0 e-02$ & 0. \\
\hline 0. & $5.0 e-03$ & 0. \\
\hline $2.4 e-02$ & 2.3E-01, & 0. \\
\hline 1. $6 e+00$ & 8. $0 e-04$ & 0. \\
\hline $7.8 e-02$ & $2.0 e-04$ & 0. \\
\hline 1. $4 e-02$ & $1.0 e-03$ & 0. \\
\hline 0. & $5.0 e-04$ & 0. \\
\hline $1.1 e-02$ & 0. & 0. \\
\hline 0. & 1.0e-03, & 0. \\
\hline 0. & $1.0 \mathrm{e}-02$, & 0. \\
\hline $0 .$, & $8.0 e-03$ & 0. \\
\hline $3.5 e-01$ & $5.0 e-04$ & 0. \\
\hline 0. & $3.0 e-04$ & 0. \\
\hline $4.5 e+0$ & $5.0 e-04$ & $0 .$, \\
\hline $9.1 e+0$ & $1.3 e-05$ & 0. \\
\hline $1.3 e+0$ & 3.0e-04, & 0. \\
\hline 0 & $5.0 e-03$ & 0. \\
\hline 1. 1e+0, & 0. & 0. \\
\hline 0. & $2.0 E-02$, & 0. \\
\hline $0 .$, & $5.0 \mathrm{e}-02$ & 0. \\
\hline 0. & 4. $0 \mathrm{E}-02$ & 0. \\
\hline 0. & $4.0 E-02$ & $0 .$, \\
\hline 0. & $3.8 \mathrm{E}-02$ & $0 .$, \\
\hline
\end{tabular}

- Input File: INVNTRY.DAT

$501,1.00 \mathrm{e}+10,1.324 \mathrm{E}+06$,

$502,1.00 \mathrm{e}+10,1.324 \mathrm{E}+06$,

$503,1.00 \mathrm{E}+10,1.324 \mathrm{E}+06$,

$504,1.00 \mathrm{E}+10,1.324 \mathrm{E}+06$,

$505,1.00 \mathrm{e}+10,1.324 \mathrm{E}+06$,
0 .

$0 .$,

0.

0.

0.
$0 ., 3.42 e 0,0$.

$0 .$,

$0 ., 0 .$,

$0 ., 4.5 \mathrm{E}-02,0 .$,

0 .

0.

$0 ., 0$.

$0 ., 0 .$,
Acenaphthene

Acetone

Anthracene

Aluminum

Antimony 
$506,1.00 e+10,1.324 \mathrm{E}+06$ $507,1.00 e+10,1.324 \mathrm{E}+06$ $508,1.00 e+10,1.324 \mathrm{E}+06$ 509, 1.00e+10,1.324E+06, $510,1.00 \mathrm{E}+10,1.324 \mathrm{E}+06$, $511,1.00 \mathrm{E}+10,1.324 \mathrm{E}+06$, $512,1.00 \mathrm{e}+10,1.324 \mathrm{E}+06$, $513,1.00 e+10,1.324 \mathrm{E}+06$ $514,1.00 \mathrm{E}+10,1.324 \mathrm{E}+06$, $515,1.00 e+10,1.324 \mathrm{E}+06$ $516,1.00 \mathrm{e}+10,1.324 \mathrm{E}+06$ $517,1.00 \mathrm{E}+10,1.324 \mathrm{E}+06$, $518,1.00 \mathrm{E}+10,1.324 \mathrm{E}+06$ 519, 1.00e+10,1.324E+06, $520,1.00 e+10,1.324 \mathrm{E}+06$ $521,1.00 e+10,1.324 \mathrm{E}+06$ $522,1.00 \mathrm{E}+10,1.324 \mathrm{E}+06$, $523,1.00 \mathrm{E}+10,1.324 \mathrm{E}+06$ $524,1.00 \mathrm{e}+10,1.324 \mathrm{E}+06$, $525,1.00 \mathrm{E}+10,1.324 \mathrm{E}+06$ $526,1.00 \mathrm{E}+10,1.324 \mathrm{E}+06$, $527,1.00 \mathrm{E}+10,1.324 \mathrm{E}+06$ $528,1.00 \mathrm{E}+10,1.324 \mathrm{E}+06$ $529,1.00 \mathrm{E}+10,1.324 \mathrm{E}+06$, $530,1.00 e+10,1.324 \mathrm{E}+06$ $531,1.00 e+10,1.324 \mathrm{E}+06$ $532,1.00 e+10,1.324 \mathrm{E}+06$ $533,1.00 e+10,1.324 \mathrm{E}+06$ $534,1.00 \mathrm{e}+10,1.324 \mathrm{E}+06$ 535, 1.00e+10,1.324E+06 $536,1.00 \mathrm{E}+10,1.324 \mathrm{E}+06$ $537,1.00 \mathrm{e}+10,1.324 \mathrm{E}+06$ $538,1.00 \mathrm{e}+10,1.324 \mathrm{E}+06$ $539,1.00 \mathrm{e}+10,1.324 \mathrm{E}+06$, $540,1.00 \mathrm{E}+10,1.324 \mathrm{E}+06$ $541,1.00 \mathrm{e}+10,1.324 \mathrm{E}+6$, $542,1.00 \mathrm{E}+10,1.324 \mathrm{E}+6$, $543,1.00 \mathrm{E}+10,1.324 \mathrm{E}+6$,

\begin{tabular}{|c|c|c|c|c|}
\hline $0 .$, & 0. & $3.10 e-2$ & 0. & Arochlors- 1242 \\
\hline $0 .$, & 0. & $3.10 e-2$ & 0. & Arochlors- 1254 \\
\hline $0 .$, & 0. & $3.10 e-2$ & 0. & Arochlors-1248 \\
\hline 0. & 0. & $3.10 e-2$ & 0. & Arochlors -1260 \\
\hline 0. & 0. & 0. & 0. & Arsenic \\
\hline 0. & 0. & 0 & 0. & Barium \\
\hline 0. & 0. & 0. & 0. & Benzene \\
\hline 0. & 0. & $5.70 e-3$ & $0 .$, & BenzoAanthracene \\
\hline 0. & 0. & 0. & $0 .$, & Beryllium \\
\hline 0. & 0. & 0. & $0 .$, & Bis2ethylhexapht \\
\hline 0. & 0. & 0. & 0. & Butylbenzylphtha \\
\hline 0. & 0. & 0. & $0 .$, & Cadmium \\
\hline 0. & 0. & 0. & $0 .$, & Calcium \\
\hline 0. & 0. & 1. $8 \mathrm{E}+00$ & $0 .$, & Carbazole \\
\hline 0. & 0. & 0. & $0 .$, & Carbontetchl \\
\hline 0. & 0. & 0. & $0 .$, & Chloroform \\
\hline 0. & 0. & 0. & $0 .$, & Chromium III \\
\hline 0. & 0. & 0. & $0 .$, & Chromium VI \\
\hline 0. & 0. & 0. & $0 .$, & Cobalt \\
\hline 0. & 0. & 0. & 0. & Copper \\
\hline 0. & 0. & 1. $4 e-02$, & 0. & Benzobflranthene \\
\hline 0. & 0. & 4.3E-03, & 0. & BenzoKflranthene \\
\hline 0. & 0. & $7.0 e-04$ & 0.1 & Benzoghiperylene \\
\hline 0. & 0. & 1.2E-03, & 0. & Benzo (a) pyrene \\
\hline 0. & 0. & $5.0 e-04$ & 0. & Dibenzoahanthrac \\
\hline 0. & 0. & 0. & 0. & Dibenzofuran \\
\hline 0. & 0. & 0. & 0. & Dieldrin \\
\hline 0. & 0. & 0. & 0. & Dinbutylphthalat \\
\hline 0. & 0 & 0. & 0. & Dinoctylphthalat \\
\hline 0. & 0. & $2.4 e+02$ & 0. & 24-dinitrotoluen \\
\hline 0. & 0. & $2.61 e-1$ & 0. & Fluoranthene \\
\hline 0. & 0. & $1.69 e+0$ & 0. & Flourene \\
\hline 0. & 0. & 0. & 0. & 2-Hexanone \\
\hline 0. & 0. & $5.3 e-04$ & 0. & Indeno123cdpyren \\
\hline 0. & 0. & 0. & 0. & Iron \\
\hline 0. & $0 .$, & 0. & 0. & Isophorone \\
\hline 0. & 0. & 0. & 0. & Lead \\
\hline 0. & $0 .$, & 0. & 0. & Lithium \\
\hline
\end{tabular}




\begin{tabular}{|c|c|c|c|c|c|c|}
\hline 544, & $1.00 \mathrm{E}+10,1.324 \mathrm{E}+6$ & 0. & 0. & 0. & 0.1 & Magnesium \\
\hline 545, & $1.00 \mathrm{E}+10,1.324 \mathrm{E}+6$ & 0. & 0. & $0 .$, & 0. & Manganese \\
\hline 546, & $1.00 \mathrm{e}+10,1.324 \mathrm{E}+6$, & 0. & 0. & $0 .$, & 0. & Mercury \\
\hline 547 & $1.00 \mathrm{e}+10,1.324 \mathrm{E}+6$ & 0. & 0. & 1.30E+04, & 0. & Methchloride \\
\hline 548 & $1.00 e+10,1.324 \mathrm{E}+6$, & 0. & 0. & $0 .$, & 0. & 2Methylnaphthale \\
\hline 549, & $1.00 \mathrm{E}+10,1.324 \mathrm{E}+6$ & 0. & 0. & $0 .$, & 0. & Naphthalene \\
\hline 550 & $1.00 \mathrm{E}+10,1.324 \mathrm{E}+6$ & 0. & 0. & $0 .$, & 0. & Nickel \\
\hline 551, & $1.00 \mathrm{E}+10,1.324 \mathrm{E}+6$, & 0. & 0. & $0 .$, & 0. & NnitroNpropyl \\
\hline 552, & $1.00 e+10,1.324 \mathrm{E}+6$ & 0. & 0. & $0 .$, & 0. & Pentachloropheno \\
\hline 553, & 1. $00 \mathrm{E}+10,1.324 \mathrm{E}+6$ & 0. & 0. & 1. $.0 \mathrm{E}+00$, & 0. & Phenanthrene \\
\hline 554, & $1.00 \mathrm{E}+10,1.324 \mathrm{E}+6$ & $0 .$, & 0. & $9.3 e+04$, & 0. & Phenol \\
\hline 555, & 1. $00 \mathrm{E}+10,1.324 \mathrm{E}+6$ & 0. & 0. & $0 .$, & 0. & Potassium \\
\hline 556, & $1.00 \mathrm{E}+10,1.324 \mathrm{E}+6$ & 0. & 0. & 1. $32 \mathrm{E}-1$, & 0. & Pyrene \\
\hline 557 & $1.00 \mathrm{E}+10,1.324 \mathrm{E}+6$ & 0. & 0. & 0. & 0. & Selenium \\
\hline 558 & $1.00 \mathrm{E}+10,1.324 \mathrm{E}+6$ & 0. & 0. & 0. & 0. & Silver \\
\hline 559 & $1.00 \mathrm{E}+10,1.324 \mathrm{E}+6$ & 0. & 0. & $0 .$, & 0. & Sodium \\
\hline 560 & $1.00 \mathrm{E}+10,1.324 \mathrm{E}+6$ & 0. & 0. & 0. & 0. & strontium \\
\hline 561, & $1.00 \mathrm{e}+10,1.324 \mathrm{E}+6$ & 0. & 0. & 0. & 0. & Tetrachloroethen \\
\hline 562, & $1.00 \mathrm{E}+10,1.324 \mathrm{E}+6$ & 0. & 0. & $0 .$, & 0. & Thallium \\
\hline 563, & $1.00 \mathrm{E}+10,1.324 \mathrm{E}+6$, & 0. & 0. & $0 .$, & 0. & $\operatorname{Tin}$ \\
\hline 564, & $1.00 \mathrm{e}+10,1.324 \mathrm{E}+6$ & 0. & 0. & 0. & $0 .$, & Toluene \\
\hline 565 & $1.00 \mathrm{e}+10,1.324 \mathrm{E}+6$, & 0. & 0. & 0. & 0. & Trichloroethene \\
\hline 566 & $7.20 \mathrm{E}+01,1.324 \mathrm{E}+6$, & 0. & 0. & 0. & 0. & $U-232$ \\
\hline 567, & $1.59 \mathrm{E}+05,1.324 \mathrm{E}+6$, & 0. & 0. & 0. & $0 .$, & $U-233$ \\
\hline 568 & $2.44 \mathrm{E}+05,1.324 \mathrm{E}+6$, & 0. & 0. & 0. & 0. & $U-234$ \\
\hline 569, & $7.04 \mathrm{E}+08,1.324 \mathrm{E}+6$ & 0. & 0. & 0. & $0 .$, & $U-235$ \\
\hline 570, & $2.34 \mathrm{E}+07,1.324 \mathrm{E}+6$ & $0 .$, & 0. & 0. & 0. & $U-236$ \\
\hline 571, & $4.47 \mathrm{E}+09,1.324 \mathrm{E}+6$ & 0. & 0. & 0. & 0. & $U-238$ \\
\hline 572, & $1.00 \mathrm{E}+10,1.324 \mathrm{E}+6$ & $0 .$, & 0. & 0. & 0. & Vanadium \\
\hline 573 & $1.00 \mathrm{E}+10,1.324 \mathrm{E}+6$ & 0. & 0. & $0 .$, & 0. & Zinc \\
\hline 574, & $1.00 \mathrm{E}+10,1.324 \mathrm{E}+6$ & 0. & 0. & 0. & 0. & Zirconium \\
\hline 575 & $1.00 \mathrm{e}+10,1.324 \mathrm{E}+6$ & 0. & 0. & 0. & 0. & $12378 \mathrm{PeCDF}$ \\
\hline 576, & $1.00 \mathrm{e}+10,1.324 \mathrm{E}+6$ & 0. & 0. & 0. & 0. & $23478 \mathrm{PeCDF}$ \\
\hline 577, & $1.00 \mathrm{e}+10,1.324 \mathrm{E}+6$ & $0 .$, & $0 .$, & 0. & 0. & OCDD \\
\hline 578 & $1.00 \mathrm{e}+10,1.324 \mathrm{E}+6$ & 0. & $0 .$, & 0. & 0. & OCDF \\
\hline 579, & $1.00 \mathrm{e}+10,1.324 \mathrm{E}+6$, & 0. & 0. & 0. & 0. & $1234678 \mathrm{HpCDD}$ \\
\hline 580 & $1.00 \mathrm{e}+10,1.324 \mathrm{E}+6$ & 0. & $0 .$, & 0. & 0. & $1234678 \mathrm{HpCDF}$ \\
\hline 581, & $1.00 \mathrm{e}+10,1.324 \mathrm{E}+6$ & 0. & 0. & 0. & 0. & $123478 \mathrm{HxCDF}$ \\
\hline
\end{tabular}


$582,1.00 \mathrm{e}+10,1.324 \mathrm{E}+6$, $583,1.00 \mathrm{e}+10,1.324 \mathrm{E}+6$, $584,1.00 \mathrm{e}+10,1.324 \mathrm{E}+6$, $585,1.00 \mathrm{e}+10,1.324 \mathrm{E}+6$ $586,1.00 \mathrm{e}+10,1.324 \mathrm{E}+6$, $590,1.00 \mathrm{e}+10,1.324 \mathrm{E}+6$, $591,1.00 \mathrm{e}+10,1.324 \mathrm{E}+6$, $592,1.00 \mathrm{E}+10,1.324 \mathrm{E}+06$ $593,1.00 \mathrm{E}+10,1.324 \mathrm{E}+06$ $594,1.00 \mathrm{E}+10,1.324 \mathrm{E}+06$, $595,1.00 \mathrm{E}+10,1.324 \mathrm{E}+06$ $592,1.00 \mathrm{E}+10,1.324 \mathrm{E}+06$ $598,1.00 \mathrm{E}+10,1.324 \mathrm{E}+06$ $619,1.00 \mathrm{E}+10,1.324 \mathrm{E}+06$ $620,1.00 \mathrm{E}+10,1.324 \mathrm{E}+06$ $621,1.00 \mathrm{E}+10,1.324 \mathrm{E}+06$ $622,1.00 \mathrm{E}+10,1.324 \mathrm{E}+06$, $623,1.00 \mathrm{E}+10,1.324 \mathrm{E}+06$ $624,1.00 \mathrm{E}+10,1.324 \mathrm{E}+06$ $625,1.00 \mathrm{E}+10,1.324 \mathrm{E}+06$ $626,1.00 \mathrm{E}+10,1.324 \mathrm{E}+06$ $627,1.00 \mathrm{E}+10,1.324 \mathrm{E}+06$, $628,1.00 \mathrm{E}+10,1.324 \mathrm{E}+06$ $629,1.00 \mathrm{E}+10,1.324 \mathrm{E}+06$ $631,1.00 \mathrm{E}+10,1.324 \mathrm{E}+06$, $632,1.00 \mathrm{E}+10,1.324 \mathrm{E}+06$ $633,1.00 \mathrm{E}+10,1.324 \mathrm{E}+06$ $634,1.00 \mathrm{E}+10,1.324 \mathrm{E}+06$ $635,1.00 \mathrm{E}+10,1.324 \mathrm{E}+06$ $636,1.00 \mathrm{E}+10,1.324 \mathrm{E}+06$ $637,1.00 \mathrm{E}+10,1.324 \mathrm{E}+06$ $638,1.00 \mathrm{E}+10,1.324 \mathrm{E}+06$ $639,1.00 \mathrm{E}+10,1.324 \mathrm{E}+06$ $640,1.00 \mathrm{E}+10,1.324 \mathrm{E}+06$, $641,1.00 \mathrm{E}+10,1.324 \mathrm{E}+06$ $642,1.00 \mathrm{E}+10,1.324 \mathrm{E}+06$, $643,1.00 \mathrm{E}+10,1.324 \mathrm{E}+06$, $644,1.00 \mathrm{E}+10,1.324 \mathrm{E}+06$,

\begin{tabular}{|c|c|c|c|}
\hline 0. & $0 .$, & $0 ., 0 .$, & $12378 \mathrm{HxCDD}$ \\
\hline 0. & $0 .$, & $0 ., 0 .$, & $123678 \mathrm{HxCDD}$ \\
\hline 0. & 0. & $0 ., 0 .$, & $123789 \mathrm{HxCDD}$ \\
\hline 0. & 0. & $0 ., 0$. & $2378 \mathrm{TCDD}$ \\
\hline 0. & 0. & $0 ., 0 .$, & $2378 \mathrm{TCDF}$ \\
\hline 0 & 0. & $8.6 e+01,0 .$, & Hexachlorethene \\
\hline 0. & 0. & $3.0 e+02,0 .$, & Trichlorobenzene \\
\hline 0. & 0. & $5.7 e+01,0 .$, & 124Trichlorb \\
\hline 0. & 0. & $7.66 \mathrm{E}+04,0$. & Molybdenum \\
\hline 0. & 0. & $0.00 E+00,0 .$, & Strontium \\
\hline 0. & 0. & $1.61 \mathrm{E}+01,0 .$, & Acenaphthylene \\
\hline 0. & 0. & $3.22 \mathrm{E}+02, \quad 0 .$, & benzidine \\
\hline 0. & 0. & $0.056,0 .$, & Chlordane \\
\hline 0. & 0. & $6.10 e+01,0 .$, & MethylPropylB \\
\hline 0. & 0. & $5.70 e+01,0 .$, & 124TriMethylB \\
\hline 0. & $0 .$, & $2.00 e+01,0 .$, & 135TrimethylB \\
\hline 0. & 0. & $6.10 e+01,0 .$, & MethylethylB \\
\hline 0. & 0. & $6.10 e+01,0 .$, & Buyt IB \\
\hline 0. & 0. & $2.20 e+02,0$. & 12DimethylB \\
\hline 0. & $0 .$, & $6.10 e+01,0 .$, & PropylB \\
\hline 0. & $0 .$, & $0.017,0 .$, & Aldrin \\
\hline 0. & 0. & $0.27,0 .$, & Aroclor 1016 \\
\hline 0. & 0. & $4.83,0$. & Aroclor 1221 \\
\hline 0. & 0. & $0.09,0$. & $\mathrm{DDD}$ \\
\hline 0. & $0 .$, & $0.04,0$. & DDE \\
\hline 0. & 0. & $0.0055,0 .$, & DDT \\
\hline 0. & 0. & $8 ., 0 .$, & Alpha-BHC \\
\hline 0. & 0. & $8 ., 0$. & Beta-BHC \\
\hline 0. & 0. & $8 ., 0 \ldots$ & Delta-BHC \\
\hline 0. & 0. & $1.61 e+01,0 .$, & Acenaphthylene \\
\hline 0. & 0. & $3.40 e+03,0 .$, & Benzolic Acid \\
\hline 0. & 0. & $3.1 e+00,0.1$ & Dibenzofuran \\
\hline 0. & 0. & $1.08 e+03,0 .$, & Diethylphth \\
\hline 0. & 0. & $7.87 e+03,0$. & 24-Dimethylphe \\
\hline 0. & 0. & $4.0 e+03,0 .$, & Dimethylphth \\
\hline 0. & 0. & $2.46 e+01,0 .$, & 2Methylnaptha \\
\hline 0. & 0. & $2.3 e+01,0 .$, & 2346 Tetrachlor \\
\hline 0. & $0 .$, & $4.29 e+04,0 .$, & Benzyl Alcohol \\
\hline
\end{tabular}


$645,1.00 \mathrm{E}+10,1.324 \mathrm{E}+06$, $646,1.00 \mathrm{E}+10,1.324 \mathrm{E}+06$, $647,1.00 \mathrm{E}+10,1.324 \mathrm{E}+06$ $648,1.00 \mathrm{E}+10,1.324 \mathrm{E}+06$ $649,1.00 \mathrm{E}+10,1.324 \mathrm{E}+06$, $650,1.00 \mathrm{E}+10,1.324 \mathrm{E}+06$ $651,1.00 \mathrm{E}+10,1.324 \mathrm{E}+06$, $652,1.00 \mathrm{E}+10,1.324 \mathrm{E}+06$ $653,1.00 \mathrm{E}+10,1.324 \mathrm{E}+06$ $654,1.00 \mathrm{E}+10,1.324 \mathrm{E}+06$ $655,1.00 \mathrm{E}+10,1.324 \mathrm{E}+06$ $656,1.00 \mathrm{E}+10,1.324 \mathrm{E}+06$ $657,1.00 \mathrm{E}+10,1.324 \mathrm{E}+06$ $658,1.00 \mathrm{E}+10,1.324 \mathrm{E}+06$, $659,1.00 \mathrm{E}+10,1.324 \mathrm{E}+06$ $660,1.00 \mathrm{E}+10,1.324 \mathrm{E}+06$ $661,1.00 \mathrm{E}+10,1.324 \mathrm{E}+06$ $662,1.00 \mathrm{E}+10,1.324 \mathrm{E}+06$ $664,1.00 \mathrm{E}+10,1.324 \mathrm{E}+06$, $665,1.00 \mathrm{E}+10,1.324 \mathrm{E}+06$ $666,1.00 \mathrm{E}+10,1.324 \mathrm{E}+06$ $667,1.00 \mathrm{E}+10,1.324 \mathrm{E}+06$, $668,1.00 \mathrm{E}+10,1.324 \mathrm{E}+06$ $669,1.00 \mathrm{E}+10,1.324 \mathrm{E}+06$ $670,1.00 \mathrm{E}+10,1.324 \mathrm{E}+06$ $671,1.00 \mathrm{E}+10,1.324 \mathrm{E}+06$, $672,1.00 \mathrm{E}+10,1.324 \mathrm{E}+06$ $673,1.00 \mathrm{E}+10,1.324 \mathrm{E}+06$ $674,1.00 \mathrm{E}+10,1.324 \mathrm{E}+06$, $675,1.00 \mathrm{E}+10,1.324 \mathrm{E}+06$ $676,1.00 \mathrm{E}+10,1.324 \mathrm{E}+06$, $677,1.00 \mathrm{E}+10,1.324 \mathrm{E}+06$ $678,1.00 \mathrm{E}+10,1.324 \mathrm{E}+06$ $679,1.00 \mathrm{E}+10,1.324 \mathrm{E}+06$, $680,1.00 \mathrm{E}+10,1.324 \mathrm{E}+06$ $682,1.00 \mathrm{E}+10,1.324 \mathrm{E}+06$ $683,1.00 \mathrm{E}+10,1.324 \mathrm{E}+06$, $684,1.00 \mathrm{E}+10,1.324 \mathrm{E}+06$
$0 ., \quad 0 ., 1.00 E+06,0 ., \quad$ Acentonitrile

$0 ., \quad 0 ., .12 \mathrm{E}+05,0 .$, Acrolien

$0 ., \quad 0 ., 7.45 \mathrm{E}+04,0 .$, Acylonitrle

$0 ., 0 ., 3.03 E+03,0 .$, Bromodichloro

$0 ., \quad 0 ., .10 \mathrm{E}+03,0 .$, Bromoform

$0 ., 0 \ldots, 52 \mathrm{E}+04,0 .$, Bromometh

$0 ., \quad 0 ., 1.18 \mathrm{E}+03,0 .$, Carbondis

$0 ., \quad 0 ., 5.32 \mathrm{E}+03,0 .$, Chlorometh

$0 ., \quad 0 ., 3.74 \mathrm{E}+02,0 ., 0-\mathrm{ChloroTu}$

$0 ., \quad 0 ., 6.13 E+01,0 .$, Cumene

$0 ., \quad 0 ., 2.70 E+03,0 .$, Dibromochloro

$0 ., \quad 0 ., 2.80 \mathrm{E}+02,0 .$, Dichlorodiflo

$0 ., \quad 0 ., 3.50 \mathrm{E}+03,0 ., 12 \mathrm{cisDichloro}$

$0 ., \quad 0 ., 3.50 \mathrm{E}+03,0 ., 12$ transDichl

$0 ., \quad 0 ., 2.80 E+03,0 .$, 12Dichlprop

$0 ., \quad 0 ., 1.69 \mathrm{E}+02,0 .$, Ethylbenz

$0 ., \quad 0 ., 9.50 E+00,0 .$, Nhexane

$0 ., 0 ., 1.90 \mathrm{E}+04,0 .$, MethylIso

$0 ., \quad 0 ., 1.00 \mathrm{E}+06,0 .$, Propglycol

$0 ., \quad 0 ., 3.10 \mathrm{E}+02,0 .$, styrene

$0 ., \quad 0 ., 1.07 \mathrm{E}+03,0 ., 1112$ Tetra

$0 ., \quad 0 ., 2.87 \mathrm{E}+03,0 ., 1122$ Tetra

0., $0 ., 1.10 \mathrm{E}+03,0 .$, Trichloflo

$0 ., \quad 0 ., 1.75 \mathrm{E}+03,0 ., 123$ Trichlopr

$0 ., \quad 0 ., 5.70 \mathrm{E}+01,0 .$, Trimethbenz

$0 ., \quad 0 ., 4.82 \mathrm{E}+01,0 ., 135 \mathrm{Trimeth}$

$0 ., \quad 0 ., 1.06 \mathrm{E}+02,0 .$, oxylene

$0 ., \quad 0 ., 6.13 E+03,0 .$, acetophenone

$0 ., \quad 0 ., 6.70 \mathrm{E}+03,0 .$, Ethylchlorid

$0 ., \quad 0 ., 1.75 \mathrm{E}+04,0 ., 2$ Hexanone

$0 ., \quad 0 ., 1.00 E+06,0 .$, Methonal.

$0 ., \quad 0 ., 1.50 e+04,0 ., \quad M M e t a c r y l a t e$

$0 ., \quad 0 ., 4.98 e+02,0 ., \quad$ Chlorobenzene

$0 ., \quad 0 ., 1.00 e+06,0 ., \quad$ Cyanide

$0 ., \quad 0 ., 1.03 e-03,0 ., \quad$ Dibenz $[\mathrm{a}, \mathrm{h}]$

$0 ., \quad 0 ., 3.52 e+02,0 ., 26$ Dinitrotoluene

$0 ., \quad 0 ., 2.00 e-02,0 ., \quad$ DiNoctylphtalate

$0 ., \quad 0.4 .50 e-01,0 .$, Endosulfan 
$685,1.00 \mathrm{E}+10,1.324 \mathrm{E}+06$, $686,1.00 \mathrm{E}+10,1.324 \mathrm{E}+06$, $687,1.00 \mathrm{E}+10,1.324 \mathrm{E}+06$ $688,1.00 \mathrm{E}+10,1.324 \mathrm{E}+06$ $689,1.00 \mathrm{E}+10,1.324 \mathrm{E}+06$, $690,1.00 \mathrm{E}+10,1.324 \mathrm{E}+06$ $691,1.00 \mathrm{E}+10,1.324 \mathrm{E}+06$ $692,1.00 \mathrm{E}+10,1.324 \mathrm{E}+06$ $693,1.00 \mathrm{E}+10,1.324 \mathrm{E}+06$ $694,1.00 \mathrm{E}+10,1.324 \mathrm{E}+06$, $695,1.00 \mathrm{E}+10,1.324 \mathrm{E}+06$ $696,1.00 \mathrm{E}+10,1.324 \mathrm{E}+06$ $697,1.00 \mathrm{E}+10,1.324 \mathrm{E}+06$, $698,1.00 \mathrm{E}+10,1.324 \mathrm{E}+06$ 699, 1.00E+10,1.324E+06 $700,1.00 \mathrm{E}+10,1.324 \mathrm{E}+06$ $701,1.00 \mathrm{E}+10,1.324 \mathrm{E}+06$, $702 ， 1.00 \mathrm{E}+10,1.324 \mathrm{E}+06$, $703,1.00 \mathrm{E}+10,1.324 \mathrm{E}+06$ $704,1.00 \mathrm{E}+10,1.324 \mathrm{E}+06$, $705,1.00 \mathrm{E}+10,1.324 \mathrm{E}+06$ $706,1.00 \mathrm{E}+10,1.324 \mathrm{E}+06$, $707,1.00 \mathrm{E}+10,1.324 \mathrm{E}+06$ $708,1.00 \mathrm{E}+10,1.324 \mathrm{E}+06$ $709,1.00 \mathrm{E}+10,1.324 \mathrm{E}+06$, $710,1.00 \mathrm{E}+10,1.324 \mathrm{E}+06$ $711,1.00 \mathrm{E}+10,1.324 \mathrm{E}+06$ $712,1.00 \mathrm{E}+10,1.324 \mathrm{E}+06$ $713,1.00 \mathrm{E}+10,1.324 \mathrm{E}+06$ $714,1.00 \mathrm{E}+10,1.324 \mathrm{E}+06$ $715,1.00 \mathrm{E}+10,1.324 \mathrm{E}+06$ $716,1.00 \mathrm{E}+10,1.324 \mathrm{E}+06$ $717,1.00 \mathrm{E}+10,1.324 \mathrm{E}+06$ $718,1.00 \mathrm{E}+10,1.324 \mathrm{E}+06$ $719,1.00 \mathrm{E}+10,1.324 \mathrm{E}+06$, $720,1.00 \mathrm{E}+10,1.324 \mathrm{E}+06$ $721,1.00 \mathrm{E}+10,1.324 \mathrm{E}+06$ $722,1.00 \mathrm{E}+10,1.324 \mathrm{E}+06$

\begin{tabular}{|c|c|c|c|c|}
\hline 0. & 0. & $1.07 e-05$ & 0. & 4Nitrobenzenamin \\
\hline 0. & 0. & $1.16 \mathrm{e}+04$ & 0. & 4Nitrophenol \\
\hline 0. & 0. & $3.50 e+01$ & 0. & NNitrosodiphen \\
\hline $0 .$, & 0. & 1. $06 \mathrm{E}+02$, & 0. & Xylene \\
\hline 0. & 0. & 8. $00 \mathrm{E}+01$ & 0. & 12Dichloro \\
\hline 0. & 0. & 1. $25 E+02$, & 0. & 13Dichloro \\
\hline 0. & 0. & 8. $13 \mathrm{E}+01$, & $0 .$, & 14Dichloro \\
\hline 0. & 0. & 1.40E+01, & 0. & Methylcyclo \\
\hline 0. & 0. & 2. 6E-04, & 0. & Benzo $[g, h, i]$ \\
\hline 0. & $0 .$, & $5.3 E+03$ & 0. & MethylChlor \\
\hline 0. & 0. & $2.5 \mathrm{E}+01$, & 0. & 2MethylNap \\
\hline 0. & 0. & 2. $5 E+03$, & 0. & 2Nitrophenol \\
\hline 0. & 0. & 0.45 , & 0. & EndosufanII \\
\hline 0. & 0. & 0.031 & 0. & Aroclor 1268 \\
\hline 0. & 0. & 0.25 & 0. & Aldehyde \\
\hline 0. & 0. & 0.25, & 0. & Ketone \\
\hline 0. & 0. & $4.98 e+02$ & 0. & Chlorobenzene \\
\hline $0 .$, & 0. & $8.8 e+03$ & 0. & Vinyl Chloride \\
\hline $0 .$, & 0. & $2.59 e+04$ & 0. & o-cresol \\
\hline 0. & $0 .$, & $2.27 e+04$ & 0. & m-cresol \\
\hline 0. & 0. & $2.15 e+04$, & 0. & p-cresol \\
\hline 0. & 0. & $8.13 e+01$ & 0. & 14Dichlorobenzen \\
\hline 0. & 0. & $6.2 e-03$ & 0. & Hexachlorobenzen \\
\hline 0. & 0. & $3.2 e+00$, & 0. & Hexachlorobutadn \\
\hline 0. & 0. & $5.0 e+01$ & 0. & Hexachloroethane \\
\hline 0. & 0. & $2.09 e+03$ & 0. & Nitrobenzene \\
\hline 0. & 0. & $8.0 e+02$ & 0. & 246-Trichlorophnl \\
\hline 0. & 0. & 1.0e+06, & 0. & Pyridine \\
\hline 0. & 0. & $6.82 \mathrm{e}+02$ & 0. & $24-D$ \\
\hline 0. & 0. & $2.0 e+02$, & 0. & 245-TP (Silvex) \\
\hline 0. & 0. & $5.6 e-02$ & 0. & Chlordane \\
\hline 0. & 0. & $2.5 e-01$ & 0. & Endrin \\
\hline 0. & 0. & $1.8 e-01$ & 0. & Heptachlor \\
\hline 0. & 0. & 2.0e-01, & 0. & Heptachlor epoxd \\
\hline 0. & 0. & $8.0 e+00$ & 0. & Lindane \\
\hline $0 .$, & 0. & $1.0 \mathrm{e}-01$ & 0. & Methoxychlor \\
\hline 0. & 0. & $5.5 e-01$ & 0. & Toxaphene \\
\hline $0 .$, & 0. & $1.76 \mathrm{e}+05$ & 0. & Lithium \\
\hline
\end{tabular}


$723,1.00 \mathrm{E}+10,1.324 \mathrm{E}+06$,

$724,1.00 \mathrm{E}+10,1.324 \mathrm{E}+06$,

$725,1.00 \mathrm{E}+10,1.324 \mathrm{E}+06$,

$726,1.00 \mathrm{E}+10,1.324 \mathrm{E}+06$

- Input File: RQSITE.DAT

$501, \quad-92.9 .2,92$,

$502,-0.044,0 ., 0.044$,

$503,-280 ., 28.0,280 .$,

$504,-353 ., \quad 35.3, \quad 353 .$,

505, -19., 1.9, 19.,

$506,-130 ., 13.0,130 .$,

$507,-11000,1100,11000$.

$508,-5600.560 .5600$.,

$509,-1.3 e 5,1.3 e 4,1.3 e 5$,

$510, \quad-29 ., \quad 1.9, \quad 19 .$,

$511, \quad-55 ., \quad 5.5,55 .$,

$512, \quad-1.7, \quad 0 ., \quad 1.7$,

$513,-2.8 e 4,2.8 e 3,2.8 e 4$,

$514,-1.4 \mathrm{e} 3,1.4 \mathrm{e} 2,1.4 \mathrm{e} 3$

515, -2.0e6, 2.0e4, 2.0e6,

$516,-2.0 \mathrm{E} 6$

$0 ., 2.0 \mathrm{e} 6$,

$517,-420$.

$42.0,420$. ,

$518,-400$.

$0 ., 400$.

$519,-6.78, \quad .678,6.78$.

$520, \quad-2.2, \quad 0 ., \quad 2.2$,

$521,-0.62$

$0 ., 0.62$,

0 .

$0 ., 5.70 e+01,0 .$,

$0 ., \quad 0 ., 5.90 e+03,0$.

$0 ., \quad 0 ., 5.90 e+03,0$.

$0 ., 0 ., 6.13 E+01,0$.

24 trimethylb

Ihexanol

2 hexanone

butylbenzene

523, -560., 56., 560., Chromium VI

$524,-3.97 e 6, \quad 0 ., 800 .$, Cobalt

525, $-30.0, .419,4.19$, Copper

$526,-1.1 e 4,1.1 e 3,1.1 e 4$, Benzobflranthene

$527,-1.1 e 4,1.1 e 3,1.1 e 4$, BenzoKflranthene

528, -1.1e4, 1.1e4, 1.1e5, Benzoghiperylene

$529,-1.1 e 5,1.1 e 4,1.1 e 5$, Benzo(a) pyrene

$530,-6.6 e 4,6.6 e 3,6.6 e 4$, Dibenzoahanthrac

$531,0.001,0 ., 0 .$, Dibenzofuran 


\begin{tabular}{|c|c|c|c|c|}
\hline 532, & $-34 \ldots$ & $0 .$, & 34. & Dieldrin \\
\hline 533, & $-1 . E-6$ & 0. & 1. E-6, & Dinbutylphthalat \\
\hline 34 & $-7.2 \mathrm{e} 7$ & $0 .$, & $7.2 \mathrm{e} 7$ & Dinoctylphthalat \\
\hline 35 & -0.9 & 0.09 & 0.9, & 24-dinitrotoluen \\
\hline 536, & -760. & 76. & 760. & Fluoranthene \\
\hline 537 & -150 & 15. & $150 .$, & Flourene \\
\hline 538, & -0.3 & $0 .$, & 0.3, & 2-Hexanone \\
\hline 539, & $-3.2 \mathrm{e} 4$ & $3.2 \mathrm{e} 3$ & $3.2 e 4$ & Indeno123cdpyren \\
\hline 540 & -1000. & 100 & 1000. & Iron \\
\hline 541 & -1.7 & 0. & 1.7, & Isophorone \\
\hline 542 & -100 & 10. & $100 \ldots$ & Lead \\
\hline 543 & -0.8 & $0 .$, & 0.8, & Lithium \\
\hline 54 & -400 & $0 .$, & 400. & Magnesium \\
\hline 545 & $-200 \ldots$ & 20. & $200 \ldots$ & Manganese \\
\hline 546 & $-580 \ldots$ & $58 \ldots$ & $580 .$, & Mercury \\
\hline 547 & -2010 & $0 .$, & 2010. & Methchloride \\
\hline 548 & $-90 \ldots$ & $0 .$, & 90. & 2Methylnaphthale \\
\hline 549 & $-19 .$, & 1.9, & 19. & Naphthalene \\
\hline 550 & -1000 & $100 \ldots$ & $1000 \ldots$ & Nickel \\
\hline 551 & -0.3 & 0.03 & 0.3, & NnitroNpropyl \\
\hline 552 & -1100 & 0. & 1100. & Pentachloropheno \\
\hline 553 & -280 & $0 .$, & 280. & Phenanthrene \\
\hline 554 & -0.28 & 0.028 & 0.28, & Phenol \\
\hline 555 & -3.98 & 0. & 30.0, & Potassium \\
\hline 556 & -760. & 76. & 760. & Pyrene \\
\hline 557 & -15 & 1.5 , & 15. & Selenium \\
\hline 558 & $-420 \ldots$ & 42. & 420. & Silver \\
\hline 559 & -10 & 0. & 10. & Sodium \\
\hline 560 & $-13 \cdot 5$ & 0. & 13.5, & Strontium \\
\hline 561 & -7.2, & 0. & 7.2, & Tetrachloroethen \\
\hline 562 & 1. & 0. & $0 .$, & Thallium \\
\hline 563 & -2.5 & .25 & 2.5 & Tin \\
\hline 564 & -6. & 0. & $6 .$, & Toluene \\
\hline 565 & -2.6 & 0. & 2.6 & Trichloroethene \\
\hline 566 & -40.0 & 0.7, & 20.0 & $\mathrm{U}-232$ \\
\hline 567 & -40.0 & 0.7, & 20.0 & $\mathrm{U}-233$ \\
\hline 568 & -40.0 & 0.7, & 20.0 & $\mathrm{U}-234$ \\
\hline 569 & -40.0 & 0.7, & 20.0 & $U-235$ \\
\hline
\end{tabular}




\begin{tabular}{|c|c|c|c|c|}
\hline 570, & -40.0 & 0.7, & 20.0, & $U-236$ \\
\hline 571, & -40.0 & 0.7, & 20.0 & $U-238$ \\
\hline 572, & -100. & 10. & 100. & Vanadium \\
\hline 573, & -940. & 94. & 940. & Zinc \\
\hline 574, & -50. & 0. & 50. & zirconium \\
\hline 575, & 1. & 0. & 0. & $12378 \mathrm{PeCDF}$ \\
\hline 576, & 1. & 0. & 0. & $23478 \mathrm{PeCDF}$ \\
\hline 577, & 1. & 0. & 0. & OCDD \\
\hline 578, & 1. & $0 .$, & $0 .$, & OCDF \\
\hline 579, & 1. & 0. & 0. & $1234678 \mathrm{HpCDD}$ \\
\hline 580 & 1., & 0. & $0 .$, & $1234678 \mathrm{HpCDF}$ \\
\hline $581,-$ & -1.40 e 6 & 0.1 & 1. 40 e6, & $123478 \mathrm{HxCDF}$ \\
\hline $582,-$ & $-1.46 e 6$ & 0.1 & 1. $46 \mathrm{e} 6$ & $12378 \mathrm{HxCDD}$ \\
\hline $583,-$ & $-1.46 \mathrm{e} 6$ & 0.1 & 1. $46 \mathrm{e} 6$, & $123678 \mathrm{HxCDD}$ \\
\hline $584,-$ & $-1.46 e 6$ & $0 ., 1$ & 1. $46 \mathrm{e} 6$ & $123789 \mathrm{HxCDD}$ \\
\hline $585,-$ & $-1.50 \mathrm{e} 6$ & 0.1 & 1.50e6, & $2378 \mathrm{TCDD}$ \\
\hline 586, & $1 .$, & 0. & 0. & $2378 \mathrm{TCDF}$ \\
\hline 590, & -0.08 & 0.008, & 0.08, & Hexachloroethene \\
\hline 591, & -0.49 & 0.049 & 0.49 & Trichlorobenzene \\
\hline 592, & -1.44, & 0.144 &, 1.44 & 124Trichlorb \\
\hline 593, & -20.0 & 2.0 & $0,20.0$ & Molybdenum \\
\hline 594, & -0.07 & 0.007 &, 0.07 & Strontium \\
\hline 595, & -122. & 12.2, &, 122. & Acenaphthylene \\
\hline 596, & -5.48 & 0.548 & 5.48 & benzidine \\
\hline 597, & -173. & 17.3， & 173. & Chlordane \\
\hline 619, & -1.65 & 0.165 & 1.65, & MethylPropylB \\
\hline 620, & -1.40 & 0.140 & 1.40, & 124 TrimethylB \\
\hline 621, & $-3 \cdot 34$ & 0.340 & 3.34, & 135TrimethylB \\
\hline 622, & -1.65 & 0.165 & 1.65, & MethylethylB \\
\hline 623, & -1.65 & 0.165 & 1.65, & Buyt IB \\
\hline 624, & -0.48 & 0.048 & 0.48, & 12DiMethylB \\
\hline 625 & -1.65 & 0.165 & 1.65, & PropylB \\
\hline 626 & -97.4 & 9.74 & 97.4 & Aldrin \\
\hline 627 & -3600. & $3600 ., 3$ & 3600. & Aroclor 1016 \\
\hline 628, & -120. & 120,1 & $120 .$, & Aroclor 1221 \\
\hline 629 & -15.0 & $15.0,1$ & 15.0, & Aroclor 1232 \\
\hline 630 & -91.6 & 9.16 & 91.6, & DDD \\
\hline 631, & -1.73 & .173, & 1.73 & $\mathrm{DDE}$ \\
\hline
\end{tabular}




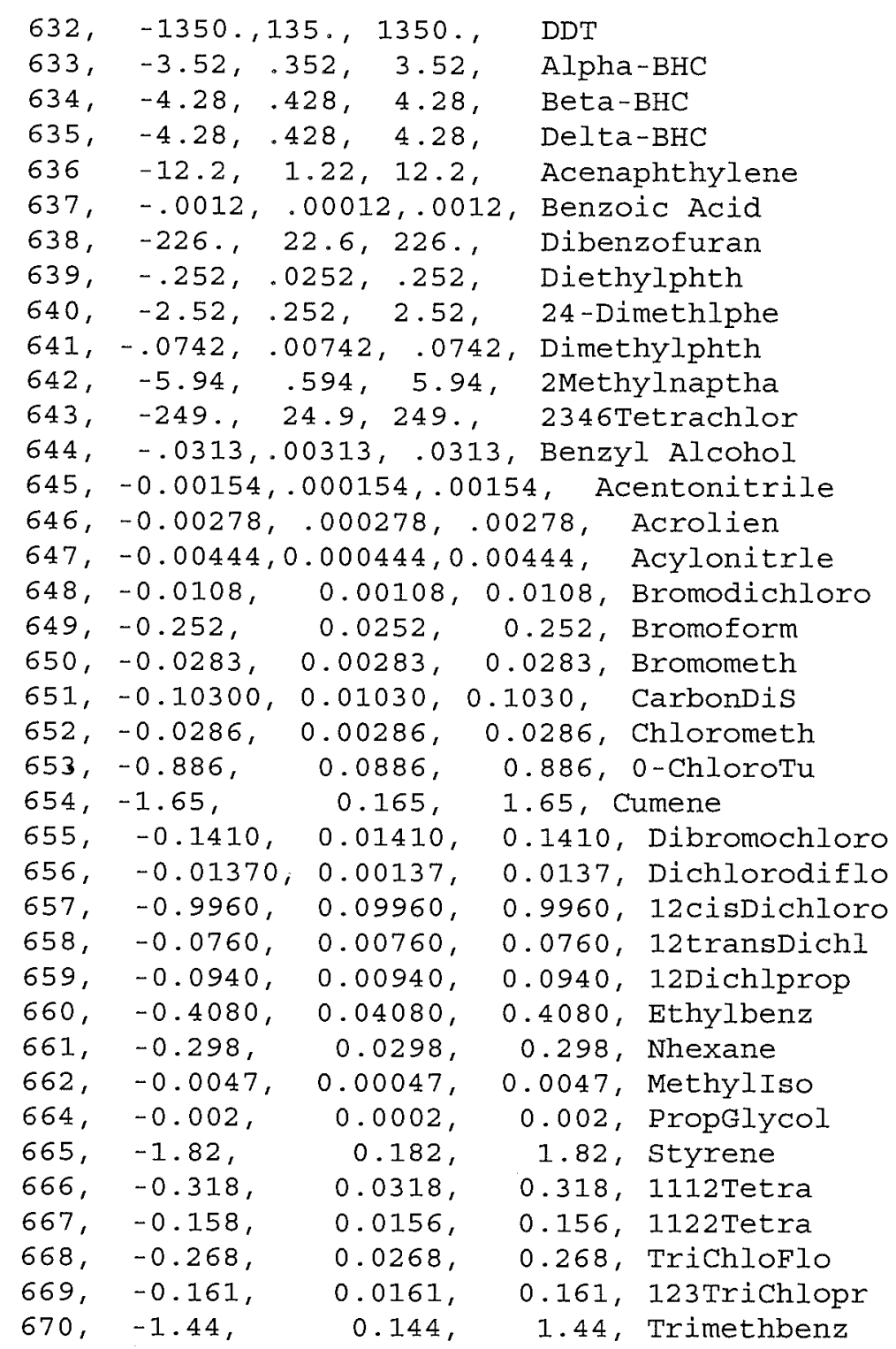




\begin{tabular}{|c|c|c|c|c|}
\hline 671, & -3.34 & 0.334, & 3.34, & 135Trimeth \\
\hline 672, & -0.482 & 0.0462 , & 0.462 , & oxylene \\
\hline 673, & -.0924 & .00924 & .0924, & acetophenone \\
\hline 674, & -0.0475 & 0.00475 & 0.0475 , & Ethylchlorid \\
\hline 675, & -0.026 & 0.0026 , & 0.026 , & 2 Hexanone \\
\hline 676, & -0.002, & 0.0002, & 0.002, & Methonal \\
\hline 677, & -0.020 & 0.0020 & 0.020 & MMetacrylate \\
\hline 678, & -0.4800 & 0.048, & 0.48, & Chlorobenzene \\
\hline 679, & -9.9 & 0.99, & 9.9, & Cyanide \\
\hline 680, & -3580.0 & 358.0, & 3580.0, & Dibenz $[\mathrm{a}, \mathrm{h}]$ \\
\hline 682, & -0.0839 & 0.00839, & 0.0839 & 26Dinitrotoluene \\
\hline 683 & -1810000 & $0,181000.0$ & 0,1810000 & 0, DiNoctylphtalate \\
\hline 684, & -4.08 & 0.408 & 4.08, & Endosulfan \\
\hline 685, & -0.344 & 0.0344, & 0.344 & 4Nitrobenzenamin \\
\hline 686, & -0.874 & 0.0874 & 0.874 & 4Nitrophenol \\
\hline 687, & -0.654 & 0.0654 , & 0.654, & NNitrosodiphen \\
\hline 688, & -.886 & 0.0886 & .886 & Xylene \\
\hline 689, & -0.758 & 0.0758, & 0.758 & 12Dichloro \\
\hline 690 & -16.06 & 1.606 , & 16.06 & 13Dichloro \\
\hline 691, & -1.232 & 0.1232, & 1.232 , & 14Dichloro \\
\hline 692, & -.199 & $0 .$, & 0. & Methylcyclo \\
\hline 693, & $-5.4 e+3$ & $5.4 e+2$, & $5.4 E+3$ & Benzo $[g, h, i]$ \\
\hline 694, & $-2.8 E-2$ & $2.8 e-3$ & $2.8 e-2$ & Methylchlor \\
\hline 695, & $-6.0 e+0$ & $6.0 e-1$ & $6.0 e+0$ & 2MethylNap \\
\hline 696 & $-7.1 e-1$ & $7.1 e-2$, & $7.1 e-1$ & 2Nitrophenol \\
\hline $697,-$ & $1.30 \mathrm{E}+05$ & 1.30E+05, & $1.30 \mathrm{E}+05$, & Aroclor 1268 \\
\hline $698,-$ & $4.08 \mathrm{E}+00$ & $4.08 E+00$ & $4.08 \mathrm{E}-01$ & EndosulfanII \\
\hline $699,-$ & $2.16 \mathrm{E}+01$ & $2.16 \mathrm{E}+01$, & $2.16 \mathrm{E}+00$ & Aldehyde \\
\hline $700,-$ & $2.16 \mathrm{E}+01$, & $2.16 \mathrm{E}+01$, & $2.16 \mathrm{E}+00$ & Ketone \\
\hline 701, & -.438 & .0438 & .438 & Chlorobenzene \\
\hline 702, & -.372, & .0372 & .372, & Vinyl Chloride \\
\hline 703, & -.182 & .0182 , & .182, & o-cresol \\
\hline 704, & -.0956 & .00956, & .0956 & m-cresol \\
\hline 705, & -.0922 & .00922, & .0922 & $\mathrm{p}$-cresol \\
\hline 706, & -1.232, & .1232, & 1.232, & 14Dichlorobenzen \\
\hline 707, & -110. & 11. & 110. & Hexachlorobenzen \\
\hline 708, & -9.35 & 0.935, & 9.35, & Hexachlorobutadn \\
\hline 709, & -3.56 & .356, & 3.56, & Hexachloroethane \\
\hline
\end{tabular}




$\begin{array}{lllll}710, & -.129, & .0129, & .129, & \text { Nitrobenzene } \\ 711, & -.636, & .0636, & .636, & 246-\text { Trichlorophnl } \\ 712, & -.0138, & 00138, & .0138, & \text { Pyridine } \\ 713, & -.0588, & .00588, & .0588, & 24-\mathrm{D} \\ 714, & -.1608, & 01608, & 1608, & 245-\mathrm{TP} \text { (Silvex) } \\ 715, & -102.6, & 10.26, & 102.6, & \text { Chlordane } \\ 716, & -21.6, & 2.16, & 21.6, & \text { Endrin } \\ 717, & -48.0, & 4.8, & 48.0, & \text { Heptachlor } \\ 718, & -17.3, & 1.73, & 17.3, & \text { Heptachlor epoxd } \\ 719, & -6.76, & 0.676, & 6.76, & \text { Lindane } \\ 720, & -160 ., & 16 ., & 160 ., & \text { Methoxychlor } \\ 721, & -198.6, & 19.86, & 198.6, & \text { Toxaphene } \\ 722, & -300 ., & 30 ., & 300 ., & \text { Lithium } \\ 723, & -1.440, & 0.1440, & 1.440, & \text { l24trimethylb } \\ 724, & -0.026, & 0.0026, & 0.026, & \text { Ihexanol } \\ 725, & -0.026, & 0.0026, & 0.026, & \text { 2hexanone } \\ 726, & -1.630, & 0.1630, & 1.630, & \text { butylbenzene }\end{array}$

- Input File: UPTAKE.DAT

\begin{tabular}{|c|c|c|c|c|c|}
\hline 0.5 , & 0.2 , & 1.89 & & & \\
\hline 0.67, & 0.65, & 2.1E-3, & 438. & 438. & \\
\hline 0.0, & 2160 & 24. & 1440. & 1. & 0.83 \\
\hline $50 \ldots$ & 6.1 & 48. & 480. & 48. & \\
\hline .05 , & 0.0008 & 60. & 8. & 50. & \\
\hline 14. & 176. & 110. & $0 .$, & 95. , & $730 ., 6.9$ \\
\hline
\end{tabular}

Acenaphthene $\quad .25,1.2 e-1,1.2 e-2,1.6 e-4,0 ., 5.0 e-4,1.1 e+3$

$.25,1.3 e+1,1.3 e+0,1.5 \mathrm{E}-8$

Anthracene $\quad .25,1.1 \mathrm{e}-1,1.1 \mathrm{E}-3,2.0 \mathrm{E}-4$

Aluminum $\quad .25,4.0 \mathrm{e}-3,4.0 \mathrm{e}-4,2.0 \mathrm{E}-4$,

Antimony $\quad .25,5.0 e-2,5.0 e-3,2.5 e-5$,

Arochlors $-1242 \quad .25,1.6 e-1,1.6 e-2,9.9 e-5$

Arochlors-1254 .25, 1.3e-2, 1.3e-3, 7.9e-3,

Arochlors-1248 .25, 1.6e-1, $1.6 e-2,5.0 e-3$,

Arochlors-1260 .25, 2.9e-3, 2.9e-4, 9.9e-2,

Arsenic

$.25,4.0 \mathrm{e}-2,4.0 \mathrm{E}-3,6.0 \mathrm{E}-5$,

$\begin{array}{ll}\text { Barium } & .25,1.0 \mathrm{e}-1,1.0 \mathrm{E}-2,4.8 \mathrm{E}-4, \\ \text { Benzene } & .25,5.8 \mathrm{E}-1,5.8 \mathrm{E}-2,3.3 \mathrm{E}-6,\end{array}$

$\begin{array}{ll}\text { Barium } & .25,1.0 \mathrm{e}-1,1.0 \mathrm{E}-2,4.8 \mathrm{E}-4, \\ \text { Benzene } & .25,5.8 \mathrm{E}-1,5.8 \mathrm{E}-2,3.3 \mathrm{E}-6,\end{array}$

$0 ., 1.5 E-8,1.5 E-8$

$0 ., 6.3 e-4,1.3 e+3$

$0 ., 1.5 e-3,0.0 e+0$

$0 ., 4.0 e-5,1.0 e+2$

$0 ., 3.1 e-4,7.7 e+2$

$0 ., 2.5 e-2,2.1 e+4$

$0 ., 1.6 e-2,1.5 e+4$

$0 ., 3.1 e-1,1.5 e+5$

$0 ., 2.0 e-3,0.0 e+0$

$0 ., 2.0 e-4,4.0 e+0$

$0 ., 3.3 E-6,3.3 E-6$

BenzoAanthracene .25, 1.9e-2, 1.9E-3,4.0E-3, 0., 1.3e-2, $1.3 e+4$ 
Berylitium

$.25,1.0 \mathrm{e}-2,1.0 \mathrm{E}-3,9.0 \mathrm{e}-7$,

Bis2ethylhexapht .25, 5.5e-2, 5.5e-3, 6.3e-4,

Butylbenzylphtha .25, 5.5e-2, 5.5e-3, 6.3e-4,

Cadmium

$25,5.5 e-1,5.5 \mathrm{E}-2,1.0 \mathrm{e}-3$

Calcium $\quad 25,3.6 e-2,3.6 \mathrm{E}-3,8.0 \mathrm{e}-3$,

Carbazole

$25,2.4 e-1,2.4 e-2,5.0 e-5$,

Carbontetchl

Chloroform

Chromium III

Chromium VI

Cobalt

Copper

$.25,2.9 e-1,2.9 e-2,1.1 e-5$

$.25,7.0 e-1,7.0 e-2,2.3 e-6$,

$.25,4.0 \mathrm{e}-2,4.0 \mathrm{E}-3,1.0 \mathrm{e}-5$

$.25,4.0 \mathrm{e}-2,4.0 \mathrm{E}-3,1.0 \mathrm{e}-5$,

$.25,9.4 e-3,9.4 e-4,5.0 e-4$

$.25,8.0 \mathrm{e}-1,8.0 \mathrm{E}-2,1.5 \mathrm{e}-3$,

Benzobflranthene $.25,1.1 \mathrm{e}-2,1.1 \mathrm{e}-3,9.9 \mathrm{e}-3$

Benzokflranthene .25, 4.3e-3, 4.3E-4, 5.0e-2,

Benzoghiperylene .25, 5.6e-3, 5.6e-4, 3.1e-2,

Benzo(a)pyrene $.25,1.1 e-2,1.1 \mathrm{E}-3,9.9 \mathrm{e}-3$,

Dibenzoahanthrac .25, 4.3e-3, 4.3e-4, 5.0e-2,

Dibenzofuran $\quad .25,0.0 e+0,0.0 e+0,0.0 e+0$,

Dieldrin $\quad 25,9.2 e-2,9.2 e-3,7.9 e-3$,

Dinbutylphthalat .25, 5.6e-3, 5.6e-4, 3.2e-3,

Dinoctylphthalat .25, $0.0 e+0,0.0 e+0,0.0 e+0$,

24 -dinitrotoluen .25, 2.6e+0, 2.6e-1, 7.9e-7,

Fluoranthene $\quad .25,5.5 e-2,5.5 \mathrm{E}-3,6.3 e-4$,

Flourene $\quad .25,1.1 \mathrm{e}-1,1.1 \mathrm{e}-2,2.0 \mathrm{e}-4$

2-Hexanone $\quad 25,5.9 e-1,5.9 e-2,2.0 e-7$,

Indeno123cdpyren .25, 5.6e-3, 5.6e-4, 3.1e-2,

Iron $\quad .25,1.0 e-2,1.0 e-3,3.0 e-5$,

Isophorone $\quad .25,4.8 \mathrm{E}-1,4.8 \mathrm{E}-2,4.6 \mathrm{e}-6$

Lead

Lithium

Magnesium

Manganese

Mercury

MethChoride

2Methylnaphthale

Naphthalene

Nickel

Nnitronpropyl
$0 ., 1.0 e-3,1.0 e+2$

$0 ., 2.0 e-3,3.1 e+3$

$0 ., 2.0 e-3,3.1 e+3$

$0 ., 4.0 e-4,2.0 E+2$

$0 ., 4.0 \mathrm{E}-3,0.0 \mathrm{e}+0$

$0 ., 1.6 e-4,4.5 e+2$

$0 ., 1.1 e-5,1.1 e-5$

$0 ., 2.3 e-6,2.3 e-6$

$0 ., 9.0 e-3,2.0 e+2$

$0 ., 9.0 e-3,2.0 e+2$

$0 ., 1.0 \mathrm{e}-3$

$0 ., 9.0 E-3,5.1 e+4$

$0 ., 3.1 e-2,2.5 e+4$

$0 ., 1.6 e-1,8.7 e+4$

$0 ., 1.0 e-1,6.1 e+4$

$0 ., 3.1 e-2,2.5 e+4$

$0 ., 1.6 e-1,8.7 e+4$

$0 ., 0.0 e+0,0.0 e+0$

$0 ., 7.9 e-3,7.9 e-3$

$0 ., 1.0 e-2,0.0 e+0$

$0 ., 0.0 e+0,0.0 e+0$

$0 ., 2.5 e-6,1.9 e+1$

$0 ., 2.0 e-3,3.1 e+3$

$0 ., 6.3 e-4,1.3 e+3$

$0 ., 6.3 e-7,6.8 e+0$

$0 ., 1.0 e-1,6.1 e+4$

$0 ., 2.0 \mathrm{e}-2,2.0 \mathrm{e}+2$

$0 ., 4.6 e-6,4.6 e-6$

$0 ., 4.0 \mathrm{e}-4,3.0 \mathrm{e}+2$

0 .

$0 ., 5.0 e-3,0.0 e+0$

$0 ., 5.0 e-4,4.0 e+2$

$0 ., 1.0 e-2,1.0 e+3$

$0 ., 5.0 e-7,0.0 e+0$

$0 ., 0.0 e+0,0.0 e+0$

$0 ., 5.0 e-5,1.9 e+2$

$0 ., 5.0 e-3,1.0 e+2$

$0 ., 6.3 e-7,6.8 e+0$ 
Pentachloropheno .25, 1.2E-2, 1.2E-3, 2.5e-3 Phenanthrene $\quad 25,8.2 \mathrm{E}-2,8.2 \mathrm{E}-3,3.1 \mathrm{e}-4$, Phenol

Potassium

Pyrene

Selenium

Silver

Sodium

Strontium

$\begin{array}{ll}\text { Strontium } & 25,1.1 \mathrm{E}+0,1.1 \mathrm{E}-1,2.8 \mathrm{E}-3 \text {, } \\ \text { Tetrachloroethen } .25,3.0 \mathrm{e}-1,3.0 \mathrm{e}-2,1.0 \mathrm{e}-5 \text {, }\end{array}$

Thallium

Tin

Toluene

Trichloroethene

$\mathrm{U}-232$

$\mathrm{U}-233$

$\mathrm{U}-234$

$\mathrm{U}-235$

$\mathrm{U}-236$

$\mathrm{U}-238$

Vanadium

Zinc

Zirconium

$12378 \mathrm{PeCDF}$

$23478 \mathrm{PeCDF}$

OCDD

$\mathrm{OCDF}$

$1234678 \mathrm{HpCDD}$

$1234678 \mathrm{HpCDF}$

$123478 \mathrm{HxCDF}$

$12378 \mathrm{HxCDD}$

$123678 \mathrm{HxCDD}$

$123789 \mathrm{HxCDD}$

$2378 \mathrm{TCDD}$

$2378 \mathrm{TCDF}$

Hexachloroethene

Trichlorobenzene

124 Trichlorb
$25,5.1 \mathrm{E}+0,5.1 \mathrm{E}-1,2.5 \mathrm{e}-7$

$25,3.7 \mathrm{E}-1,3.7 \mathrm{E}-2,1.0 \mathrm{E}-2$

$.25,5.5 e-2,5.5 E-3,6.3 e-4$,

$25,5.0 e-1,5.0 e-2,1.0 e-2$,

.25, 1.0e+0, 1.0E-1, 5.0e-5,

$25,2.0 \mathrm{e}-1,2.0 \mathrm{E}-2,1.6 \mathrm{e}-2$

. $25, \quad 0 ., \quad 0 ., \quad 0$.

$.25,1.0 \mathrm{e}+0,1.0 \mathrm{E}-1,1.0 \mathrm{e}-3$,

$25,2.6 e-1,2.6 e-2,1.3 e-5$,

$.25,4.1 e-1,4.1 e-2,6.0 e-6$,

$25,2.3 e-2,2.3 \mathrm{E}-3,4.0 \mathrm{e}-4$,

$.25,2.3 e-2,2.3 E-3,4.0 e-4$,

$.25,2.3 e-2,2.3 E-3,4.0 e-4$,

$.25,2.3 e-2,2.3 E-3,4.0 e-4$

$.25,2.3 e-2,2.3 \mathrm{E}-3,4.0 \mathrm{e}-4$,

$.25,2.3 e-2,2.3 \mathrm{E}-3,4.0 \mathrm{e}-4$,

$.25,5.5 e-3,5.5 \mathrm{E}-4,2.0 \mathrm{e}-5$,

$.25,9.9 e-1,9.9 \mathrm{E}-2,1.0 \mathrm{e}-2$

$.25, \quad 0 ., \quad 0 ., \quad 0$.

$.25, \quad 0.0 e+0,0.0 e+0,0.0 e+0$,

$25,0.0 e+0,0.0 e+0,0.0 e+0$

$25,3.1 e-4,3.1 e-5,4.8 e+0$,

$25,3.1 e-4,3.1 e-5,4.8 e+0$,

$.25,0.0 e+0,0.0 e+0,0.0 e+0$

$.25,0.0 e+0,0.0 e+0,0.0 e+0$,

$.25,0.0 e+0,0.0 e+0,0.0 e+0$

$.25,0.0 e+0,0.0 e+0,0.0 e+0$,

$.25,0.0 e+0,0.0 e+0,0.0 e+0$,

$.25,0.0 e+0,0.0 e+0,0.0 e+0$,

$.25,0.0 e+0,0.0 e+0,0.0 e+0$,

$.25,0.0 e+0,0.0 e+0,0.0 e+0$

$.25,2.0 e-1,4.3 e-2,6.3 e-5$,

$25,1.8 e-1,3.7 e-2,7.9 e-5$,

$.25,2.44 e-1,2.44 e+0,4.8 e-5$,
$0 ., 2.5 e-3,2.5 e-3$

$0 ., 1.0 e-3,1.8 e+3$

$0 ., 7.9 e-7,8.1 e+0$

$0 ., 1.2 \mathrm{E}-2,1.0 \mathrm{E}+3$

$0 ., 2.0 e-3,3.1 e+3$

$0 ., 1.0 e-1,0.0 e+0$

$0 ., 3.0 e-3,5.0 e+0$

$0 ., 8.0 e-2,2.0 e+1$

$0 ., 8.0 e-3,0.0 \mathrm{E}+0$

$0 ., 1.0 e-5,1.0 e-5$

$0 ., \quad 0 ., \quad 0$.

$0 ., 1.0 e-2,3.0 e+3$

$0 ., 1.3 e-5,1.3 e-5$

$0 ., 6.0 e-6,6.0 e-6$

$0 ., 3.0 e-4,1.0 e+1$

$0 ., 3.0 \mathrm{e}-4,1.0 \mathrm{e}+1$

$0 ., 3.0 \mathrm{e}-4,1.0 \mathrm{e}+1$

$0.3 .0 e-4,1.0 e+1$

$0 ., 3.0 e-4,1.0 e+1$

$0.3 .0 \mathrm{e}-4,1.0 \mathrm{e}+1$

$0 ., 2.5 e-3,1.0 e+1$

$0 ., 1.0 \mathrm{e}-1,1.0 \mathrm{e}+3$

$0 ., \quad 0 ., \quad 0$.

$0 ., 0.0 e+0,0.0 e+0$

$0 ., 0.0 e+0,0.0 e+0$

$0 ., 1.5 e+1,2.8 e+6$

$0 ., 1.5 e+1,2.8 e+6$

$0 ., 0.0 e+0,0.0 e+0$

$0 ., 0.0 e+0,0.0 e+0$

$0 ., 0.0 e+0,0.0 e+0$

$0 ., 0.0 e+0,0.0 e+0$

$0 ., 0.0 e+0,0.0 e+0$

$0 ., 0.0 e+0,0.0 e+0$

$0 ., 0.0 e+0,0.0 e+0$

$0 ., 0.0 e+0,0.0 e+0$

$0 ., 2.0 e-4,5.4 e+2$

$0 ., 2.5 e-4,6.5 e+2$

$0 ., 1.5 e-04,0.0 e+0$ 
Molybdenum

strontium

Acenaphthylene

benzidine

Chlordane

MethylpropylB

124 TrimethylB

135TrimethylB

MethylEthylB

ButylB

12DiMethylB

PropylB

Aldrin

Aroclor 1016

Aroclor 1221

Aroclor 1232

DDD

DDE

Alpha-BHC

Beta-BHC

Delta-BHC

Acenaphthylene

Benzoic Acid

Dibenzofuran

Diethylphth

24-Dimethylphe

Dimethylphth

2Methylnaptha

2346 Tetrachlor

Benzyl Alcohol

Acentonitrile

Acrolien

Acylonitrle

Bromodichloro

Bromoform

Bromometh

Carbondis
$25,4.0 e-1,4.0 e-2,1.7 e-3$ $25,1.1 e+0,1.1 e-1,2.8 e-3$, $.25,2.7 e-1,2.7 e-2,4.0 e-5$, $25,6.7 e+0,6.7 e-1,1.6 e-7$ $.25,2.5 E-2,2.5 E-3,2.5 E-3$, $25,3.5 e-1,3.5 e-2,2.5 e-5$ $25,4.1 e-1,4.1 e-2,2.1 e-5$, $.25,3.9 e-1,3.9 e-2,2.1 e-5$ $25,3.5 e-1,3.5 e-2,2.5 e-5$, $.25,3.5 e-1,3.5 e-2,2.5 e-5$, $25,6.0 e-1,6.0 e-2,1.1 e-5$, $.25,3.5 e-1,3.5 e-2,2.5 e-5$ $25,6.9 e-1,6.9 e-2,7.9 e-6$ $25,1.4 e-2,1.4 e-3,6.3 e-7$, $.25,1.6 e-1,1.6 e-2,9.9 e-5$, $25,5.3 e-1,5.3 e-2,1.3 e-5$ $.25,1.6 e-2,1.6 e-3,5.0 e-3$, $25,1.9 e-2,1.9 e-3,4.0 e-3$ $.25,7.8 e-3,7.8 e-4,1.8 e-2$ $.25,2.1 e-1,2.1 e-2,6.3 e-5$ $25,1.8 e-1,1.8 e-2,7.9 e-5$ $25,9.0 e-1,9.0 e-2,5.0 e-6$, $25,2.7 e-1,2.7 e-2,4.0 e-5$ $25,3.0 e+0,3.0 e-1,6.3 e-7$, $25,1.5 e-1,1.5 e-2,1.0 e-4$ $.25,1.3 e+0,1.3 e-1,2.5 e-6$ $.25,1.8 e+0,1.8 e-1,1.6 e-6$, $.25,4.5 e+0,4.5 e-1,3.1 e-7$ $.25,2.1 e-1,2.1 e-2,6.3 e-5$, $.25,1.6 e-1,1.6 e-2,9.9 e-5$ $.25,8.7 e+0,8.7 e-1,9.9 e-8$, $.25,6.0 e+1,6.0 e+0,3.6 e-9$ $.25,4.3 e+1,4.3 e+0,6.3 e-9$, $.25,2.7 e+1,2.7 e+0, \quad 1.4 e-8$ $.25,2.3 e+0,2.3 e-1,9.9 e-7$ $.25,1.5 \mathrm{E}+0,1.5 e-1,2.0 e-6$ $.25,7.7 \mathrm{E}+0,7.7 e-1, \quad 1.3 e-7$ $.25,2.0 e+0,2.0 e-1,1.3 e-6$,
$0.1 .0 e-03,0.0 e+0$ $0 ., 8.0 e-03,0.0 e+0$ $0 ., 1.3 e-04,0.0 e+0$ $0 ., 5.0 e-7,0.0 e+0$ $0 ., 7.9 \mathrm{E}-3,0.0 \mathrm{e}+0$ $0 ., 7.9 e-5,0.0 e+0$ $0 ., 6.6 e-5,0.0 e+0$ $0 ., 6.6 e-5,0.0 e+0$ $0 ., 7.9 e-5,0.0 e+0$ $0 ., 7.9 e-5,0.0 e+0$ $0 ., 3.4 e-5,0.0 e+0$ $0 ., 7.9 e-5,0.0 e+0$ $0 ., 2.5 e-5,0.0 e+0$ $0 ., 2.0 e-2,0.0 e+0$ $0 ., 3.1 e-4,0.0 e+0$ $0 ., 4.0 e-5,0.0 e+0$ $0 ., 1.6 e-2,0.0 e+0$ $0 ., 1.3 e-2,0.0 e+0$ $0 ., 5.7 e-2,0.0 e+0$ $0 ., 2.0 e-4,0.0 e+0$ $0 ., 2.5 e-4,0.0 e+0$ $0 ., 1.6 e-5,0.0 e+0$ $0 ., 1.3 e-4,0.0 e+0$ $0 ., 2.0 e-6,0.0 e+0$ $0 ., 3.3 e-4,0.0 e+0$ $0 ., 7.9 e-6,0.0 e+0$ $0 ., 5.0 e-6,0.0 e+0$ $0 ., 1.0 e-6,0.0 e+0$ $0 ., 2.0 e-4,0.0 e+0$ $0 ., 3.1 e-4,0.0 e+0$ $0 ., 3.1 e-7,0.0 e+0$ $0 ., 1.1 e-8,0.0 e+0$ $0 ., 2.0 e-8,0.0 e+0$ $0 ., 4.4 e-8,0.0 e+0$ $0 ., 3.1 e-6,0.0 e+0$ $0 ., 6.3 e-6,0.0 e+0$ $0 ., 4.0 e-7,0.0 e+0$ $0.4 .0 e-6,0.0 e+0$ 


\begin{tabular}{|c|c|c|c|c|c|c|}
\hline hlorometh & .25 & 1. $1 e+1$ & $1.1 e+0$ & $6.4 e-8$ & $0 ., 2.0 \mathrm{e}-7$ & $0 \mathrm{e}+0$ \\
\hline 0 -ChloroTu & 25, & 4.1E-1, & 4.1e-2, & $2.0 e-5$ & $0 ., 6.3 e-5$, & $0.0 e+0$ \\
\hline Cumene & 25, & $3.5 E-1$ & 3. $5 e-2$, & $2.5 e-5$ & $7.9 e-5$, & $0.0 e+0$ \\
\hline ibromochloro & 25, & $2.0 E+0$ & $2.0 \mathrm{e}-1$ & 1. $3 e-6$, & -6 & $0.0 e+0$ \\
\hline rodiflo & 25, & 2. $0 \mathrm{E}+0$ & $2.0 e-1$ & 1. $3 e-6$, & $4.0 e-6$ & $0.0 e+0$ \\
\hline ichloro & .25, & $3.0 e+0$ & $3.0 e-1$ & $6.3 e-7$ & $2.0 e-6$ & $0.0 e+0$ \\
\hline Dichl & .25 & $2.0 e+1$ & $2.0 e+0$ & $2.4 e-8$ & $0 ., 7.5 e-8$, & $0.0 e+0$ \\
\hline lprop & 25, & $2.6 \mathrm{e}+0$ & $2.6 e-1$ & $7.9 e-7$ & $0 ., 2.5 e-6$, & $e+0$ \\
\hline enz & .25, & $6.1 \mathrm{e}-1$ & $6.1 e-2$, & $9.9 e-6$ & $0 ., 3.1 e-5$ & $0.0 e+0$ \\
\hline e & .25, & $2.1 \mathrm{e}-1$ & $2.1 e-2$, & $6.3 e-5$ & $2.0 e-4$ & $0.0 e+0$ \\
\hline so & .25, & $7.7 e+0$ & 7. $7 e-1$, & 1. $3 e-7$, & $0 ., 4.0 e-7$ & $0.0 e+0$ \\
\hline 1 & .25, & $3.7 e+2$ & $3.7 e+1$ & $1.6 e-10$ & $5.0 e-10$ & $0.0 e+$ \\
\hline ty & .25, & $7.9 e-1$ & $7.9 e-2$ & $6.3 e-6$ & $2.0 e-5$ & $0.0 e+0$ \\
\hline ra & .25, & $6.9 e-1$ & $6.9 e-2$ & $7.9 e-6$, & $2.5 e-5$ & $0.0 e+0$ \\
\hline ra & .25, & $1.5 e+0$ & 1. $5 e-1$ & $2.0 e-6$ & $0 ., 6.3 e-6$, & $0.0 e+0$ \\
\hline Flo & .25, & $1.3 e+0$ & $1.3 e-1$ & $2.5 e-6$, & $0 ., 7.9 e-6$ & $e+0$ \\
\hline lopr & .25, & $8.2 e-2$ & $8.2 e-3$ & 3. $1 e-4$, & 1. 0 e -3 & $0.0 e+0$ \\
\hline abenz & .25, & $2.4 \mathrm{e}-1$ & $2.4 e-2$ & $4.8 e-5$ & 1. $5 e-4$ & $0.0 e+0$ \\
\hline eth & $.25 ，$ & $3.9 e-1$ & $3.9 e-2$ & $2.1 e-5$ & $6.6 e-5$ & $0.0 e+0$ \\
\hline oxy & .25, & $5.3 e-1$ & $5.3 e-2$ & 1. $3 e-5$, & $4.0 e-5$ & $0.0 e+0$ \\
\hline lenone & .25 & $3.9 e+0$ & $3.9 e-1$ & $4.0 e-7$ & $1.3 e-6$ & $e+0$ \\
\hline orid & .25, & $5.9 e+0$ & $5.9 e-1$ & $2.0 e-7$ & $6.3 e-7$ & $0.0 e+0$ \\
\hline ne & .25, & $5.9 e+0$ & $5.9 e-1$ & $2.0 e-7$ & $6.3 e-7$ & $0.0 e+0$ \\
\hline 1 & .25 & 1. $1 \mathrm{E}+2$ & $1.1 e+1$ & 1. $3 E-9$, & 4. $2 \mathrm{E}-9$ & $0.0 e+0$ \\
\hline ylate & .25 & $6.7 e+0$ & $6.7 e-1$ & 1. $6 e-7$, & $0 ., 5.0 e-7$ & $0.0 e+0$ \\
\hline benzene & .25, & $9.0 e-1$ & $9.0 e-2$, & $5.0 e-6$ & $\Rightarrow 1.6 e-5$ & 3. $1 e+1$ \\
\hline Cya & .25, & $8.7 e+0$ & $8.7 e-1$, & $9.9 e-8$ & $0 ., 3.1 e-7$, & $3.5 e+0$ \\
\hline$[a, b]$ & .25, & $4.3 e-3$ & $4.3 e-4$ & $5.0 e-2$ & $0 ., 1.6 \mathrm{e}-1$, & 6.3 \\
\hline rotoluene & .25, & $3.9 e+0$ & $3.9 e-1$ & $4.0 e-7$ & $0 ., 1.3 e-6$, & $6.2 e+0$ \\
\hline Iphtalate & .25, & $1.8 e-4$ & 1. $8 e-5$ & $1.3 e+1$ & $0 ., 4.0 \mathrm{e}+1$, & $8.9 e+1$ \\
\hline $1 \mathrm{fan}$ & .25 & $3.3 e-1$ & $3.3 e-2$ & $2.8 e-5$ & $0 ., 8.9 e-5$, & $5.2 €$ \\
\hline enzenamin & .25 & $6.8 e+0$ & $6.8 e-1$ & $2.0 e-7$ & $0 ., 6.2 e-7$ & $9.6 e+2$ \\
\hline phenol & .25, & $3.0 e-0$ & $3.0 \mathrm{e}-1$ & $6.3 e-7$ & $0 ., 2.0 e-6$, & $3.1 e+2$ \\
\hline sodiphen & .25 & $6.1 e-1$ & $6.1 e-2$ & $9.9 e-6$ & $0 ., 3.0 e-5$, & $5.3 e+0$ \\
\hline $\mathrm{XYle}$ & .25, & $4.6 e-1$ & $4.6 e-2$ & 1. $6 e-5$, & $0 ., 5.0 e-5$, & $5.5 e+1$ \\
\hline $12 \mathrm{D}$ & .25 & 4. $1 \mathrm{e}-1$ & $4.1 e-2$ & $2.0 e-5$ & $0 ., 6.3 e-5$ & $8.7 e+1$ \\
\hline oro & .25 & $3.1 e-1$ & 3.1e-2, & $3.1 e-5$ & $0 ., 1.0 \mathrm{e}-4$ & $1.0 e+2$ \\
\hline Loro & .25, & 4.1e-1, & $4.1 e-2$ & $2.0 e-5$ & $0.6 .3 e-5$, & $8 \cdot 9 e+1$ \\
\hline
\end{tabular}




\begin{tabular}{|c|c|c|c|c|c|c|c|}
\hline Methylcyclo & .25 & 8. $3 e-1$ & 8. $3 e-2$, & $5.7 e-6$ & $0 .$, & $1.8 e-5$ & $1.2 e+2$ \\
\hline Benzo $[g, h, i]$ & .25, & $5.6 e-3$ & $5.6 e-4$ & $3.1 e-2$, & $0 .$, & 1.0e-1, & 0 . \\
\hline Methylchlor & .25, & 1. $1 e+1$ & $1.1 e+0$, & $6.4 e-8$ & $0 .$, & $2.0 \mathrm{e}-7$ & 0 \\
\hline 2 MethylNap & .25, & $2.1 e-1$ & $2.1 e-2$, & $6.3 e-5$ & 0. & $2.0 e-4$ & 0 . \\
\hline Aroclor 1268 & .25, & $2.9 e-3$ & $2.9 e-4$ & $9.9 e-2$ & $0 .$, & 3. $1 e-1$ & $0.0 e+0$ \\
\hline EndosulfanII & .25, & $3.3 e-1$ & $3.3 e-2$, & $2.8 e-5$ & $0 .$, & $8.9 e-5$ & $0.0 e+0$ \\
\hline Aldehyde & .25, & $8.2 e-2$, & $8.2 e-3$ & 3.1e-4, & $0 .$, & 1.0e-3, & $0.0 e+0$ \\
\hline Ketone & .25, & $8.2 e-2$, & $8.2 e-3$ & $3.1 e-4$ & $0 .$, & 1.0e-3, & $0.0 e+0$ \\
\hline Chlorobenzene & .25, & $9.0 e-1$ & $9.0 e-2$, & $5.0 e-6$, & $0 .$, & $1.6 e-5$ & $0.0 e+0$ \\
\hline Vinyl Chloride & .25, & $5.9 e+0$ & $5.9 e-1$ & $2.0 e-7$ & $0 .$, & $6.3 e-7$ & $0.0 e+0$ \\
\hline o-cresol & .25 & $3.0 e+0$ & $3.0 e-1$, & $6.3 e-7$ & $0 .$, & $2.0 e-6$ & $0.0 e+0$ \\
\hline m-cresol & .25, & $2.6 e+0$ & $2.6 e-1$ & $7.9 e-7$ & 0. & $2.5 e-6$ & $0.0 e+0$ \\
\hline p-cresol & .25 & $3.0 e+0$ & 3. $0 e-1$ & $6.3 e-7$ & 0. & $2.0 e-6$ & $0.0 e+0$ \\
\hline 14Dichlorobenzen & .25, & 4.1e-1, & 4.1e-2, & $2.0 e-5$ & 0. & $6.3 e-5$ & $0.0 e+0$ \\
\hline Hexachlorobenzen & .25 & $3.2 e-2$, & $3.2 e-3$ & 1. $6 e-3$, & 0. & $5.0 e-3$ & $0.0 e+0$ \\
\hline Hexachlorobutadn & .25 , & $6.2 e-2$, & $6.2 e-3$ & $5.0 e-4$ & 0. & 1. $6 e-3$, & $0.0 e+0$ \\
\hline Hexachloroethane & .25, & $2 \cdot 1 e-1$, & $2 \cdot 1 e-2$, & $6.3 e-5$ & 0. & $2.0 e-4$ & $0.0 e+0$ \\
\hline Nitrobenzene & .25, & $3.4 e+0$ & $3.4 e-1$ & $5.0 e-7$ & 0. & 1. $6 e-6$, & $0.0 e+0$ \\
\hline 246-TrichlorphnI & .25, & $2.7 e-1$ & $2.7 e-2$, & $4.0 e-5$ & 0. & 1. $3 e-4$ & $0.0 e+0$ \\
\hline Pyridine & .25 , & $6.7 e+0$, & $6.7 e-1$ & 1.6e-7, & 0. & $5.0 e-7$ & $0.0 e+0$ \\
\hline $24-D$ & .25, & 1. $3 e+0$, & 1. $3 e-1$, & $2.5 e-6$ & 0. & $7.9 e-6$ & $0.0 e+0$ \\
\hline 245-TP (Silvex) & .25, & $2.1 e-1$ & 2. 1e-2, & $6.3 e-5$ & 0. & $2.0 e-4$ & $0.0 e+0$ \\
\hline Chlordane & .25 & $2.5 e-2$, & $2.5 e-3$ & $2.5 e-3$ & 0. & $7.9 e-3$ & $0.0 e+0$ \\
\hline Endrin & .25, & $8.2 e-2$, & $8.2 e-3$ & 3. 1e-4, & 0. & $1.0 e-3$ & $0.0 e+0$ \\
\hline Heptachlor & .25, & 1.2e-1, & 1.2e-2, & 1. $6 e-4$ & 0. & $5.0 e-4$ & $0.0 e+0$ \\
\hline Heptachlor epoxd & .25 & $2.8 e-2$ & $2.8 e-3$ & $2.0 \mathrm{e}-3$ & 0. & $6.3 e-3$ & $0.0 e+0$ \\
\hline Lindane & .25 , & $2.7 e-1$ & $2.7 e-2$, & $4.0 e-5$ & 0. & $1.3 e-4$ & $0.0 e+0$ \\
\hline Methoxychlor & .25 , & 1.1e-1, & 1.1e-2, & $2.0 e-4$ & 0. & $6.3 e-4$ & $0.0 e+0$ \\
\hline Toxaphene & .25, & $6.2 e-2$, & $6.2 e-3$ & $5.0 e-4$ & 0. & 1.6e-3, & $0.0 e+0$ \\
\hline Lithium & .25 , & $2.5 e-2$ & $2.5 e-3$ & $2.0 e-2$ & 0. & $1.0 e-2$, & $0.0 e+0$ \\
\hline 124 trimethylb & .25 & $2.4 e-1$, & $2.4 e-2$ & $4.8 e-5$ & 0. & 1. $5 e-4$, & $0.0 e+0$ \\
\hline 1hexanol & .25 & $5.9 e+0$ & $5.9 e-1$ & $2.0 e-7$ & 0. & $6.3 e-7$ & $0.0 e+0$ \\
\hline 2hexanone & .25, & $5.9 e+0$ & $5.9 e-1$ & $2.0 e-7$ & 0. & $6.3 e-7$ & $0.0 e+0$ \\
\hline butylbenze & 25, & $3.5 e-1$ & $3.5 e-2$ & $2.5 e-5$ & $0 .$, & $7.9 e-5$ & $0.0 e+0$ \\
\hline
\end{tabular}


THERE ARE 202 CONTAMINANTS IN THE RISK FACTOR LIBRARY NUMBER OF TIMES FOR CALCULATION IS 2

YEARS TO BE CALCULATED ARE ...

1000.00100000 .00

THERE ARE 47 CONTAMINANTS IN THE INVENTORY FILE THE VALUE OF IFLAG IS 0

NUMBER OF PATHWAYS IS 5

PATHWAY

GROUNDWATER TO RIVER

$0 \quad 3 \mathrm{X}, \mathrm{I} 2,2 \mathrm{X}, \mathrm{A} 22,6 \mathrm{X}, \mathrm{I} 2)) \square \square$

$0 \quad 3 X, I 2,2 X, A 22,6 X, I 2)) \square \square$

$0 \quad 3 X, I 2,2 X, A 22,6 X, I 2)) \square \square$

$0 \quad 3 \mathrm{X}, \mathrm{I} 2,2 \mathrm{X}, \mathrm{A} 22,6 \mathrm{X}, \mathrm{I} 2)) \square \square$

TIME OF OPERATION OF WASTE FACILITY IN YEARS

LENGTH OF REPOSITORY (M)

WIDTH OF REPOSITORY (M)

RIVER FLOW RATE $(M * * 3 / Y R)$

STREAM FLOW RATE $(M * * 3 / Y R)$

DISTANCE TO RIVER (M)

OPERATIONAL SPILLAGE FRACTION

DENSTTY OF AQUIFER (KG/M**3)

LONGITUDINAL DISPERSIVITY (M)

LATERAL DISPERSION COEFFICIENT - - Y AXIS $(M * * 2 / Y R)$

NUMBER OF MESH POINTS FOR DISPERSION CALCULATION

FLAG FOR ATMOSPHERIC PATHWAY

COVER THICKNESS OVER WASTE (M)

THICKNESS OF WASTE IN PITS (M)

TOTAL WASTE VOLUME $(M * * 3)$
TYPE OF USAGE

FOR UPTAKE FACTORS

2
0
0
0
0

0

166

525.

$2.23 E+05$

1. $00 \mathrm{E}+00$

74 .

$0.00 E+00$ 1800 .

$6.00 \mathrm{E}+00$

$0.00 E+00$

20

0

4.00

15.30

1. $324 \mathrm{E}+00$ 
DISTANCE TO WELL - - X COORDINATE (M)

DISTANCE TO WELL - - Y COORDINATE (M)

DENSITY OF WASTE $(\mathrm{KG} / \mathrm{M} * * 3)$

0

1600 .

FRACTION OF FOOD CONSUMED THAT IS GROWN ON SITE

.400

.705

.0100

.900

1.000

1. $00 \mathrm{E}-07$

8000

.705

0 .

1. $00 \mathrm{E}+00$

240 .

$5.56 \mathrm{E}-04$

4

6.30

.2300

0

1. $10 \mathrm{E}-06$

.0100

.0

.00

.0

$0.00 \mathrm{E}+00$

HEAT EMISSION RATE FROM BURNING (CAL/S)

FLAGS FOR DEGRADATION SERIES

FLAG FOR INPUT SUMMARY PRINTOUT

FLAG FOR DIRECTION OF TRENCH FILLING

FLAG FOR GROUNDWATER PATHWAY OPTIONS

AMOUNT OF WATER PERCOLATING THROUGH WASTE ANNUALLY (M)

DEGREE OF SOIL SATURATION

RESIDUAL SOIL SATURATION

$$
\begin{aligned}
& 1 \\
& 0 \\
& 1 \\
& 9.10 \mathrm{E}-03 \\
& 1.000 \\
& .000
\end{aligned}
$$


PERMEABILITY OF VERTICAL ZONE (M/YR)

SOIL NUMBER

POROSITY OF AQUIFER

POROSITY OF UNSATURATED ZONE

DISTANCE FROM AQUIFER TO WASTE (M)

AVERAGE VERTICAL GROUNDWATER VELOCITY (M/YR)

.32

.000

.04

.25

4.6

$2.50 \mathrm{E}-02$

4. $60 \mathrm{E}+00$

10.000

1. $000 \mathrm{E}-05$

1. $000 \mathrm{E}+00$

$0.00 \mathrm{E}+00$ 


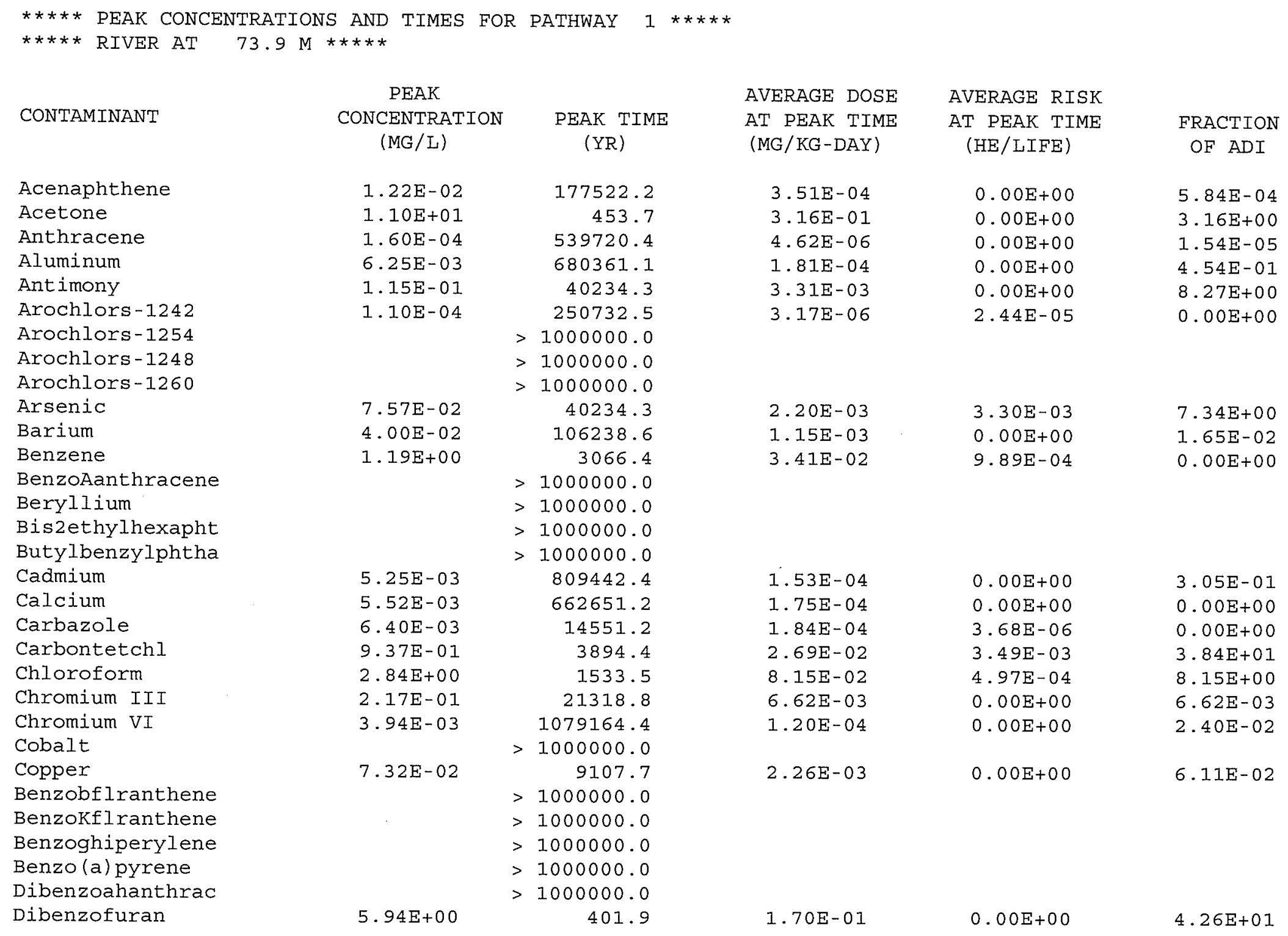


Dieldrin

Dinbutylphthalat

Dinoctylphthalat

24-dinitrotoluen

Fluoranthene

Flourene

2-Hexanone

Indeno123cdpyren

Iron

Isophorone

Lead

Lithium

Magnesium

Manganese

Mercury

MethChoride

2Methylnaphthale

Naphthalene

Nickel

NnitroNpropyl

Pentachloropheno

Phenanthrene

Phenol

Potassium

Pyrene

selenium

silver

sodium

Strontium

Tetrachloroethen

Thallium

Tin

Toluene

Trichloroethene

$\mathrm{U}-233$

$\mathrm{J}-234$

$\mathrm{U}-235$

$\mathrm{U}-236$
6. 46E-02
1. $41 \mathrm{E}+01$
$8.54 E-01$
$4.84 \mathrm{E}+00$
1. $19 \mathrm{E}+00$
2. 20E-02
2. $31 \mathrm{E}+00$
5. $52 \mathrm{E}-03$
1. $10 \mathrm{E}-02$
3. $80 \mathrm{E}-03$
1. $25 \mathrm{E}+01$
2. $45 \mathrm{E}-02$
1. $15 \mathrm{E}-01$
4. $84 E+00$
3. $56 E-03$
$5.06 E+00$
5. 34E-01
1. $46 \mathrm{E}-01$
5. 25E-03
2. 17E-01
1. $62 \mathrm{E}-01$
3. $00 \mathrm{E}-01$
1. $59 \mathrm{E}+02$
8. 31E-01
3. $59 \mathrm{E}-01$
8. $01 E-01$
4. $83 E-02$
5. $05 \mathrm{E}-02$
5. 50E-02

56555.2

401.9

$>1000000.0$

2193.0

$>1000000.0$

289264.2

897.6

5. $49 \mathrm{E}-02$
2. 10E-03
$4.46 E-01$
$2.45 E-02$
1. $73 E-04$
1. $39 E-01$
3. $41 \mathrm{E}-02$
$6.36 \mathrm{E}-04$
6. $62 \mathrm{E}-02$
1. $75 \mathrm{E}-04$
3. $17 \mathrm{E}-04$
1. $17 \mathrm{E}-04$
3. $59 \mathrm{E}-01$
7. $02 \mathrm{E}-04$
$3.31 E-03$
1. 39E-01
1. $03 \mathrm{E}-04$
1. $45 \mathrm{E}-01$
1. $81 \mathrm{E}-02$
7. $48 \mathrm{E}-03$
1. $54 \mathrm{E}-04$
1. $07 \mathrm{E}-02$
$5.02 E-03$
8. $61 z-03$
4. $56 \mathrm{E}+00$
2. 57E-02
1. 03E-02
2. 30E-02
1. $39 \mathrm{E}-03$
1. $46 \mathrm{E}-03$
1. $59 \mathrm{E}-03$
1. $58 \mathrm{E}-03$

$\begin{array}{ll}2.72 \mathrm{E}-02 & 4.19 \mathrm{E}+01 \\ 0.00 \mathrm{E}+00 & 4.46 \mathrm{E}+00 \\ 1.66 \mathrm{E}-05 & 1.22 \mathrm{E}+01 \\ 0.00 \mathrm{E}+00 & 4.34 \mathrm{E}-03 \\ 0.00 \mathrm{E}+00 & 0.00 \mathrm{E}+00\end{array}$

3. $24 \mathrm{E}-05$

1. $71 \mathrm{E}-01$

$0.00 E+00$

$0.00 E+00$

$0.00 E+00$

$0.00 \mathrm{E}+00$

$0.00 \mathrm{E}+00$

2. $69 \mathrm{E}-03$

1. $00 \mathrm{E}-05$

$0.00 \mathrm{E}+00$

$9.71 E-01$

1. 19E-03

$0.00 E+00$

$0.00 \mathrm{E}+00$

$0.00 E+00$

$0.00 \mathrm{E}+00$

$.00 E+00$

$0.00 E+00$

4. $48 E-04$

$0.00 E+00$

$0.00 \mathrm{E}+00$

$0.00 \mathrm{E}+00$

2. 53E-04

$0.00 \mathrm{E}+00$

$0.00 E+00$

$0.00 \mathrm{E}+00$

$0.00 \mathrm{E}+00$
4. 54E-01

3. $31 E+00$

$0.00 \mathrm{E}+00$

6. 75E-03

3. $91 \mathrm{E}-01$

5. $99 E+00$

1. $17 \mathrm{E}-02$

9. 18E-02

$0.00 E+00$

$0.00 E+00$

2. $42 \mathrm{E}-01$

$0.00 \mathrm{E}+00$

1. $50 \mathrm{E}+00$

3. $08 \mathrm{E}-02$

$0.00 E+00$

8. $37 \mathrm{E}-03$

8. $61 \mathrm{E}-01$

$5.69 \mathrm{E}+04$

5. 14E-02

$0.00 E+00$

4. 64 E-01

4. $86 \mathrm{E}-01$

5. $28 \mathrm{E}-01$
4. $29 \mathrm{E}-02$

5. $29 E-01$ 


U-238
Vanadium
Zinc
Zirconium
12378 PeCDF
23478 PeCDF
OCDD
OCDF
1234678HpCDD
1234678HpCDF
123478HxCD
12378HxCDD
123678HxCDD
123789HxCDD
$2378 T C D D$
2378TCDF
Hexachloroethene
124Trichlorb
Molybdenum
Strontium
Acenaphthylene
benzidine
Chlordane
Cumene
MethylPropylB
124TriMethylB
135TriMethylB
MethylEthylB
ButylB
12DiMethylB
PropylB
Aldrin
Aroclor1016
Aroclor1221
Aroclorl232
DDD
DDE
DDT

5. 50E-0
$2.20 \mathrm{E}-0$
$4.40 \mathrm{E}-0$
$1.59 \mathrm{E}+0$
$1.59 \mathrm{E}+0$
$1.59 \mathrm{E}+0$
$1.59 \mathrm{E}+0$
$1.59 \mathrm{E}+0$
$1.59 \mathrm{E}+0$

1. $59 \mathrm{E}+02$

3. $06 \mathrm{E}-01$

2. $03 \mathrm{E}-01$

1. $09 \mathrm{E}-01$

1. $62 \mathrm{E}-01$

1. $81 \mathrm{E}-02$

3. $92 \mathrm{E}-01$

1. $99 \mathrm{E}-04$

1. $22 \mathrm{E}-03$

1. $22 \mathrm{E}-03$

1. $42 \mathrm{E}-03$

6. 31E-04

1. $22 \mathrm{E}-03$

1. $22 \mathrm{E}-03$

3. $47 E-03$

1. $22 \mathrm{E}-03$

$6.05 E-05$

1. $70 \mathrm{E}-02$

1. $72 \mathrm{E}-02$

3. $20 E-04$

1. $42 \mathrm{E}-04$
34039.3

192934.9
1000000.0

83051.2

214.6

214.6

214.6

214.6

214.6

214.6

$>1000000.0$

$>1000000.0$

$>1000000.0$

$>1000000.0$

$>1000000.0$

$$
214.6
$$

626.1

3327.9

42336.1

22607.2

235319.8

11818.9

333575.7

3769.3

3769.3

3243.9

7328.1

3769.3

3769.3

1310.3

3769.3

187925.8

$>1000000.0$

543728.5

110340.9

176751.6

3937.4

$>1000000.0$
1. $59 \mathrm{E}-03$

6. $43 E-04$

1. $26 E-03$

4. $56 \mathrm{E}+00$

$4.56 \mathrm{E}+00$

$6.88 \mathrm{E}+02$

$6.88 \mathrm{E}+02$

$4.56 \mathrm{E}+00$

4. $56 \mathrm{E}+00$

$4.56 \mathrm{E}+00$

8. $79 \mathrm{E}-03$

$5.82 \mathrm{E}-03$

3. 21E-03

$5.02 \mathrm{E}-03$

5. 19E-04

1. $12 \mathrm{E}-02$

6. $16 \mathrm{E}-06$

3. 51E-05

3. $51 \mathrm{E}-05$

$4.07 \mathrm{E}-05$

1. $81 \mathrm{E}-05$

3. $51 \mathrm{E}-05$

3. $51 \mathrm{E}-05$

9. $95 \mathrm{E}-05$

3. $51 \mathrm{E}-05$

1. $73 E-06$

4. $88 \mathrm{E}-04$

4. $93 E-04$

1. $06 \mathrm{E}-05$

4. $60 \mathrm{E}-06$
$0.00 E+00$

0. $00 \mathrm{E}+00$

$0.00 E+00$

$6.83 \mathrm{E}+03$

$6.83 E+02$

$2.07 \mathrm{E}+03$

$2.07 \mathrm{E}+03$

1. $37 \mathrm{E}+02$

1. $37 \mathrm{E}+02$

1. $37 \mathrm{E}+03$

1. $23 \mathrm{E}-04$

$0.00 \mathrm{E}+00$

$0.00 \mathrm{E}+00$

$0.00 E+00$

$0.00 E+00$

2. $58 \mathrm{E}+00$

2. 16E-06

$0.00 \mathrm{E}+00$

$0.00 \mathrm{E}+00$

$0.00 E+00$

$0.00 E+00$

$0.00 E+00$

$0.00 E+00$

$0.00 E+00$

$0.00 E+00$

2. $95 E-05$

1. $95 E-04$

1. $97 \mathrm{E}-04$

2. $55 \mathrm{E}-06$

1. 57E-06
5. 29E-01

9. $19 E-02$

3. $61 z-02$

$0.00 E+00$

$0.00 \mathrm{E}+00$

$0.00 E+00$

$0.00 \mathrm{E}+00$

$0.00 E+00$

$0.00 E+00$
$0.00 E+00$

8. $79 \mathrm{E}+00$

1. 16E-01

6. $43 \mathrm{E}-01$

8. $37 \mathrm{E}-03$

$0.00 \mathrm{E}+00$

3. $74 \mathrm{E}+00$

1. $23 \mathrm{E}-02$

3. $51 \mathrm{E}-04$

9. $48 \mathrm{E}-04$

8. $14 \mathrm{E}-04$

3. $62 \mathrm{E}-04$

9. $48 \mathrm{E}-04$

9. $48 \mathrm{E}-04$

4. $98 \mathrm{E}-05$

9. $48 \mathrm{E}-04$

5. $78 \mathrm{E}-02$

$0.00 \mathrm{E}+00$

$0.00 \mathrm{E}+00$

$0.00 \mathrm{E}+00$

$0.00 \mathrm{E}+00$ 


\begin{tabular}{|c|c|}
\hline Alpha-BHC & $2.85 E-02$ \\
\hline Beta-BHC & $2.85 E-02$ \\
\hline Delta-BHC & $2.85 E-02$ \\
\hline Acenaphthylene & $5.73 E-02$ \\
\hline Benzoic Acid & $1.21 \mathrm{E}+01$ \\
\hline Dibenzofuran & $9.76 \mathrm{E}-03$ \\
\hline Diethylphth & $3.84 E+00$ \\
\hline 24-Dimethylphe & $8.25 E-01$ \\
\hline Dimethylphth & $9.58 \mathrm{E}+00$ \\
\hline 2Methylnaptha & $8.75 E-02$ \\
\hline 2346 Tetrachlor & $8.86 E-03$ \\
\hline Benzyl Alcohol & $1.18 \mathrm{E}+01$ \\
\hline Acentonitrile & $1.40 E+01$ \\
\hline Acrolien & $1.39 \mathrm{E}+01$ \\
\hline Acylonitrle & $1.37 \mathrm{E}+01$ \\
\hline Bromodichloro & $1.08 \mathrm{E}+01$ \\
\hline Bromoform & $5.41 \mathrm{E}+00$ \\
\hline Bromometh & $1.20 E+01$ \\
\hline Carbondis & 4. $20 \mathrm{E}+00$ \\
\hline Chlorometh & $1.19 \mathrm{E}+01$ \\
\hline 0 -ChloroTu & $1.33 \mathrm{E}+00$ \\
\hline Dibromochloro & $7.42 \mathrm{E}+00$ \\
\hline Dichlorodiflo & $9.96 \mathrm{E}-01$ \\
\hline 12cisDichloro & $1.92 \mathrm{E}+00$ \\
\hline 12transDichl & $9.50 \mathrm{E}+00$ \\
\hline 12Dichlprop & $8.82 E+00$ \\
\hline Ethylbenz & $6.01 E-01$ \\
\hline Nhexane & $3.38 \mathrm{E}-02$ \\
\hline MethylIso & 1.37E+01 \\
\hline MethChloride & $1.25 \mathrm{E}+01$ \\
\hline PropGlycol & $1.39 E+01$ \\
\hline styrene & $1.10 E+00$ \\
\hline 1112 Tetra & $3.81 \mathrm{E}+00$ \\
\hline 1122 Tetra & $7.02 \mathrm{E}+00$ \\
\hline Trichloflo & $3.91 E+00$ \\
\hline 123Trichlopr & $6.22 \mathrm{E}+00$ \\
\hline Trimethbenz & $2.03 E-01$ \\
\hline 135Trimeth & $1.71 \mathrm{E}-01$ \\
\hline
\end{tabular}

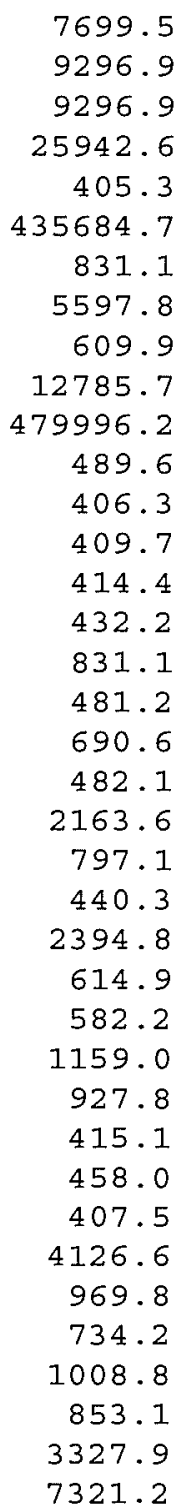

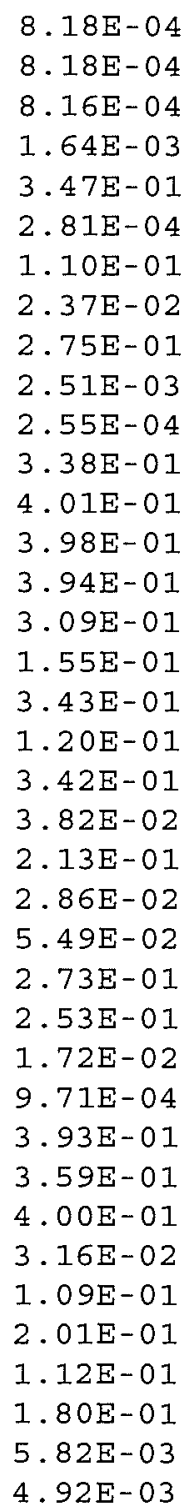

$\begin{array}{ll}5.15 E-03 & 0.00 E+00 \\ 1.47 E-03 & 0.00 E+00 \\ 1.47 E-03 & 0.00 E+00 \\ 0.00 E+00 & 2.74 E-02 \\ 0.00 E+00 & 8.67 E-02 \\ 0.00 E+00 & 7.02 E-02 \\ 0.00 E+00 & 1.38 E-01 \\ 0.00 E+00 & 1.18 E+00 \\ 0.00 E+00 & 2.75 E-02 \\ 0.00 E+00 & 6.29 E-01 \\ 0.00 E+00 & 8.49 E-03 \\ 0.00 E+00 & 1.13 E+00 \\ 0.00 E+00 & 6.69 E+01 \\ 0.00 E+00 & 7.96 E+02 \\ 2.13 E-01 & 3.94 E+02 \\ 1.92 E-02 & 1.55 E+01 \\ 1.22 E-03 & 7.75 E+00 \\ 0.00 E+00 & 2.45 E+02 \\ 0.00 E+00 & 1.20 E+00 \\ 4.45 E-03 & 0.00 E+00 \\ 0.00 E+00 & 1.91 E+00 \\ 1.79 E-02 & 1.06 E+01 \\ 0.00 E+00 & 1.43 E-01 \\ 0.00 E+00 & 5.49 E+00 \\ 0.00 E+00 & 1.36 E+01 \\ 1.72 E-02 & 0.00 E+00 \\ 0.00 E+00 & 1.72 E-01 \\ 0.00 E+00 & 1.62 E-02 \\ 0.00 E+00 & 4.92 E+00 \\ 2.69 E-03 & 5.99 E+00 \\ 0.00 E+00 & 8.01 E-01 \\ 0.00 E+00 & 1.58 E-01 \\ 2.84 E-03 & 3.64 E+00 \\ 4.03 E-02 & 3.36 E+00 \\ 0.00 E+00 & 3.74 E-01 \\ 1.26 E+00 & 3.00 E+01 \\ 0.00 E+00 & 1.16 E-01 \\ 0.00 E+00 & 9.84 E-02 \\ & \\ 0.00\end{array}$


oxylene

acetophenone

Ethylchlorid

2 Hexanone

Methonal

Metacrylate

Chlorobenzene

Cyanide

Dibenz $[\mathrm{a}, \mathrm{h}]$

26Dinitrotoluene

DiNoctylphtalate

Endosulfan

$4 \mathrm{Ni}$ trobenzenamin

$4 \mathrm{Nitrophenol}$

NNitrosodiphen

Xylene

12Dichloro

13Dichloro

14Dichloro

Methylcyclo

Benzo $[g, h, i]$

MethylChlor

2MethylNap

$2 \mathrm{Nitrophenol}$

Aroclor 1268

EndosulfanII

Aldehyde

Ketone

Chlorobenzene

vinyl Chloride

o-cresol

m-cresol

$\mathrm{p}$-cresol

14Dichlorobenzen

Hexachlorobenzen

Hexachlorobutadn

Hexachloroethane

Nitrobenzene
3. $77 \mathrm{E}-01$
$8.88 \mathrm{E}+00$
1. $08 \mathrm{E}+01$
1. $21 \mathrm{E}+01$
1. $39 \mathrm{E}+01$
1. 25E+01
1. $77 \mathrm{E}+00$
2. 19E-01
1. $25 \mathrm{E}+00$
1. $60 \mathrm{E}-03$
3. $81 \mathrm{E}-08$
2. $14 \mathrm{E}+00$
1. $24 \mathrm{E}-01$
3. $77 \mathrm{E}-01$
2. $85 \mathrm{E}-01$
1. $36 \mathrm{E}-01$
2. 89E-01
4. $98 \mathrm{E}-02$
1. $20 E+01$
8. 89E-02
$2.55 \mathrm{E}+00$
1. $60 \mathrm{E}-03$
8. 89E-04
8. 89E-04
1. $77 \mathrm{E}+00$
4. $18 \mathrm{E}+00$
$6.52 \mathrm{E}+00$
8. $76 \mathrm{E}+00$
8. $88 \mathrm{E}+00$
2. $89 \mathrm{E}-01$
2. $20 \mathrm{E}-05$
$1.14 \mathrm{E}-02$
1. $78 \mathrm{E}-01$

7. $43 \mathrm{E}+00$

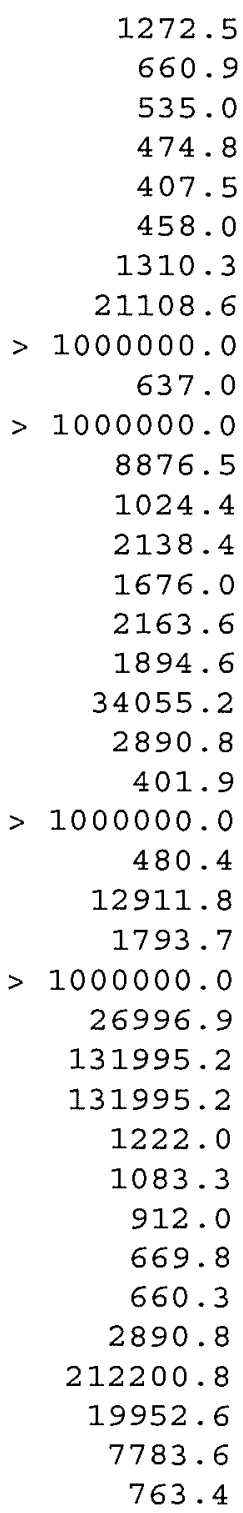

763.4

$\begin{array}{lll}1.08 E-02 & 0.00 E+00 & 5.41 E-03 \\ 2.55 E-01 & 0.00 E+00 & 2.55 E+00 \\ 3.11 E-01 & 9.01 E-04 & 7.77 E-01 \\ 3.47 E-01 & 0.00 E+00 & 8.68 E+00 \\ 4.00 E-01 & 0.00 E+00 & 8.00 E-01 \\ 3.59 E-01 & 0.00 E+00 & 2.57 E-01 \\ 5.08 E-02 & 0.00 E+00 & 2.54 E+00 \\ 6.29 E-03 & 0.00 E+00 & 3.15 E-01 \\ & & \\ 3.59 E-02 & 2.44 E-02 & 3.59 E+01 \\ 4.59 E-05 & 0.00 E+00 & 7.66 E-03 \\ 1.09 E-09 & 2.29 E-11 & 3.64 E-07 \\ 6.14 E-02 & 0.00 E+00 & 9.91 E-01 \\ 3.57 E-03 & 1.75 E-05 & 1.79 E-01 \\ 1.08 E-02 & 0.00 E+00 & 5.41 E-02 \\ 8.16 E-03 & 0.00 E+00 & 9.07 E-02 \\ 3.91 E-03 & 0.00 E+00 & 4.39 E-02 \\ 8.30 E-03 & 1.99 E-04 & 3.61 E-02 \\ 1.43 E-03 & 0.00 E+00 & 2.38 E-02 \\ 3.44 E-01 & 4.47 E-03 & \\ 2.56 E-03 & 0.00 E+00 & 0.00 E+00 \\ 7.31 E-02 & 0.00 E+00 & 6.39 E-01 \\ 4.59 E-05 & 0.00 E+00 & 1.18 E+00 \\ 2.58 E-05 & 0.00 E+00 & 7.66 E-03 \\ 2.58 E-05 & 0.00 E+00 & 8.58 E-02 \\ 5.08 E-02 & 0.00 E+00 & 8.58 E-02 \\ 1.20 E-01 & 1.68 E-01 & 2.54 E+00 \\ 1.87 E-01 & 0.00 E+00 & 3.99 E+01 \\ 2.51 E-01 & 0.00 E+00 & 3.74 E+00 \\ 2.55 E-01 & 0.00 E+00 & 5.03 E+00 \\ 8.30 E-03 & 1.99 E-04 & 5.10 E+01 \\ 6.64 E-07 & 1.06 E-06 & 3.61 E-02 \\ 3.32 E-04 & 2.59 E-05 & 8.30 E-04 \\ 5.11 E-03 & 7.15 E-05 & 5.11 E+00 \\ 2.13 E-01 & 0.00 E+00 & \\ 1.26 E+02\end{array}$


246-Trichlorphnl

Pyridine

24-D

245-TP (Silvex)

Chlordane

Endrin

Heptachlor

Heptachlor epoxd

Lindane

Methoxychlor

Toxaphene

Lithium

124 trimethylb

1hexanol

2hexanone

butylbenzene
2. $79 E+00$
1. $30 E+01$
2. $43 \mathrm{E}+00$
7. $11 \mathrm{E}-01$
1. $99 \mathrm{E}-04$
8. 89E-04
6. $40 \mathrm{E}-04$
7. $11 \mathrm{E}-04$
2. $85 \mathrm{E}-02$
3. $56 \mathrm{E}-04$
1. $96 \mathrm{E}-03$
7. $35 E-03$
2. $03 \mathrm{E}-01$
1. $21 \mathrm{E}+01$
1. $21 \mathrm{E}+01$
2. 18E-01

\begin{abstract}
8. $00 \mathrm{E}-02$
3. $72 \mathrm{E}-01$

6. $96 \mathrm{E}-02$

2. 04E-02

6. 16E-06

2. 58E-05

1. 85E-05

2. 17E-05

8.17E-04

1. $03 E-05$

5. 70E-05

2. $66 \mathrm{E}-04$

5. 82E-03

3. $47 E-01$

3. $47 \mathrm{E}-01$

6. 26E-03
\end{abstract}

$\begin{array}{ll}8.80 E-04 & 0.00 E+00 \\ 0.00 E+00 & 3.72 E+02 \\ 0.00 E+00 & 6.96 E+00 \\ 0.00 E+00 & 2.56 E+00 \\ 2.16 E-06 & 1.23 E-02 \\ 0.00 E+00 & 8.58 E-02 \\ 8.30 E-05 & 3.69 E-02 \\ 1.97 E-04 & 1.67 E+00 \\ 1.06 E-03 & 2.72 E+00 \\ 0.00 E+00 & 2.05 E-03 \\ 6.27 E-05 & 0.00 E+00 \\ 0.00 E+00 & 1.33 E-02 \\ 0.00 E+00 & 1.16 E-01 \\ 0.00 E+00 & 8.68 E+00 \\ 0.00 E+00 & 8.68 E+00 \\ 0.00 E+00 & 1.65 E-01\end{array}$


$* * * * * * * * *$ CONTAMINANT DOSES (mg $/ \mathrm{kg}$-day) $* * * * * * * *$

TIMES 1000. 100000.

$\begin{array}{lll}\text { Acenaphthene } & 0.0 \mathrm{E}+00 & 0.0 \mathrm{E}+00 \\ \text { Acetone } & 1.5 \mathrm{E}-14 & 0.0 \mathrm{E}+00 \\ \text { Anthracene } & 0.0 \mathrm{E}+00 & 0.0 \mathrm{E}+00 \\ \text { Aluminum } & 0.0 \mathrm{E}+00 & 0.0 \mathrm{E}+00 \\ \text { Antimony } & 0.0 \mathrm{E}+00 & 1.6 \mathrm{E}-20 \\ \text { Arochlors }-1242 & 0.0 \mathrm{E}+00 & 0.0 \mathrm{E}+00 \\ \text { Arochlors }-1254 & 0.0 \mathrm{E}+00 & 0.0 \mathrm{E}+00 \\ \text { Arochlors }-1248 & 0.0 \mathrm{E}+00 & 0.0 \mathrm{E}+00 \\ \text { Arochlors-1260 } & 0.0 \mathrm{E}+00 & 0.0 \mathrm{E}+00 \\ \text { Arsenic } & 0.0 \mathrm{E}+00 & 2.2 \mathrm{E}-03 \\ \text { Barium } & 1.2 \mathrm{E}-03 & 1.2 \mathrm{E}-03 \\ \text { Benzene } & 0.0 \mathrm{E}+00 & 0.0 \mathrm{E}+00 \\ \text { BenzoAanthracene } & 0.0 \mathrm{E}+00 & 0.0 \mathrm{E}+00 \\ \text { Beryllium } & 0.0 \mathrm{E}+00 & 0.0 \mathrm{E}+00 \\ \text { Bis2ethylhexapht } & 0.0 \mathrm{E}+00 & 0.0 \mathrm{E}+00 \\ \text { Butylbenzylphtha } & 0.0 \mathrm{E}+00 & 0.0 \mathrm{E}+00 \\ \text { Cadmium } & 0.0 \mathrm{E}+00 & 0.0 \mathrm{E}+00 \\ \text { Calcium } & 0.0 \mathrm{E}+00 & 0.0 \mathrm{E}+00 \\ \text { Carbazole } & 0.0 \mathrm{E}+00 & 1.8 \mathrm{E}-04 \\ \text { Carbontetchl } & 0.0 \mathrm{E}+00 & 0.0 \mathrm{E}+00 \\ \text { Chloroform } & 0.0 \mathrm{E}+00 & 0.0 \mathrm{E}+00 \\ \text { Chromium III } & 0.0 \mathrm{E}+00 & 0.0 \mathrm{E}+00 \\ \text { Chromium VI } & 0.0 \mathrm{E}+00 & 0.0 \mathrm{E}+00 \\ \text { Cobalt } & 0.0 \mathrm{E}+00 & 0.0 \mathrm{E}+00 \\ \text { Copper } & 0.0 \mathrm{E}+00 & 0.0 \mathrm{E}+00 \\ \text { Benzobflranthene } & 0.0 \mathrm{E}+00 & 0.0 \mathrm{E}+00 \\ \text { BenzoKflranthene } & 0.0 \mathrm{E}+00 & 0.0 \mathrm{E}+00 \\ \text { Benzoghiperylene } & 0.0 \mathrm{E}+00 & 0.0 \mathrm{E}+00 \\ \text { Benzo(a)pyrene } & 0.0 \mathrm{E}+00 & 0.0 \mathrm{E}+00 \\ \text { Dibenzoahanthrac } & 0.0 \mathrm{E}+00 & 0.0 \mathrm{E}+00 \\ \text { Dibenzofuran } & 1.7 \mathrm{E}-01 & 0.0 \mathrm{E}+00 \\ \text { Dieldrin } & 0.0 \mathrm{E}+00 & 2.1 \mathrm{E}-03 \\ \text { Dinbutylphthalat } & 0.0 \mathrm{E}+00 & 0.0 \mathrm{E}+00 \\ \text { Dinoctylphthalat } & 0.0 \mathrm{E}+00 & 0.0 \mathrm{E}+00\end{array}$




$\begin{array}{lll}\text { 24-dinitrotoluen } & 0.0 \mathrm{E}+00 & 0.0 \mathrm{E}+00 \\ \text { Fluoranthene } & 0.0 \mathrm{E}+00 & 0.0 \mathrm{E}+00 \\ \text { Flourene } & 0.0 \mathrm{E}+00 & 0.0 \mathrm{E}+00 \\ \text { 2-Hexanone } & 1.4 \mathrm{E}-01 & 0.0 \mathrm{E}+00 \\ \text { Indenol23cdpyren } & 0.0 \mathrm{E}+00 & 0.0 \mathrm{E}+00 \\ \text { Iron } & 0.0 \mathrm{E}+00 & 0.0 \mathrm{E}+00 \\ \text { Isophorone } & 0.0 \mathrm{E}+00 & 0.0 \mathrm{E}+00 \\ \text { Lead } & 0.0 \mathrm{E}+00 & 0.0 \mathrm{E}+00 \\ \text { Lithium } & 0.0 \mathrm{E}+00 & 0.0 \mathrm{E}+00 \\ \text { Magnesium } & 0.0 \mathrm{E}+00 & 0.0 \mathrm{E}+00 \\ \text { Manganese } & 0.0 \mathrm{E}+00 & 0.0 \mathrm{E}+00 \\ \text { Mercury } & 0.0 \mathrm{E}+00 & 0.0 \mathrm{E}+00 \\ \text { Mehylenechloride } & 0.0 \mathrm{E}+00 & 0.0 \mathrm{E}+00 \\ \text { 2Methylnaphthale } & 0.0 \mathrm{E}+00 & 0.0 \mathrm{E}+00 \\ \text { Naphthalene } & 0.0 \mathrm{E}+00 & 1.6 \mathrm{E}-20 \\ \text { Nickel } & 0.0 \mathrm{E}+00 & 0.0 \mathrm{E}+00 \\ \text { NnitroNpropyl } & 1.4 \mathrm{E}-01 & 0.0 \mathrm{E}+00 \\ \text { Pentachloropheno } & 0.0 \mathrm{E}+00 & 0.0 \mathrm{E}+00 \\ \text { Phenanthrene } & 0.0 \mathrm{E}+00 & 0.0 \mathrm{E}+00 \\ \text { Phenol } & 1.5 \mathrm{E}-01 & 0.0 \mathrm{E}+00 \\ \text { Potassium } & 0.0 \mathrm{E}+00 & 0.0 \mathrm{E}+00 \\ \text { Pyrene } & 0.0 \mathrm{E}+00 & 0.0 \mathrm{E}+00 \\ \text { Selenium } & 0.0 \mathrm{E}+00 & 0.0 \mathrm{E}+00 \\ \text { Silver } & 0.0 \mathrm{E}+00 & 0.0 \mathrm{E}+00 \\ \text { Sodium } & 0.0 \mathrm{E}+00 & 0.0 \mathrm{E}+00 \\ \text { Strontium } & 0.0 \mathrm{E}+00 & 0.0 \mathrm{E}+00 \\ \text { Tetrachloroethen } & 0.0 \mathrm{E}+00 & 0.0 \mathrm{E}+00 \\ \text { Thallium } & 0.0 \mathrm{E}+00 & 0.0 \mathrm{E}+00 \\ \text { Tin } & 0.0 \mathrm{E}+00 & 0.0 \mathrm{E}+00 \\ \text { Toluene } & 0.0 \mathrm{E}+00 & 0.0 \mathrm{E}+00 \\ \text { Trichloroethene } & 0.0 \mathrm{E}+00 & 0.0 \mathrm{E}+00 \\ \text { U-232 } & 0.0 \mathrm{E}+00 & 0.0 \mathrm{E}+00 \\ \text { U-233 } & 0.0 \mathrm{E}+00 & 1.0 \mathrm{E}-03 \\ \text { U-234 } & 0.0 \mathrm{E}+00 & 1.2 \mathrm{E}-03 \\ \text { U-235 } & 0.0 \mathrm{E}+00 & 1.6 \mathrm{E}-03 \\ \text { U-236 } & 0.0 \mathrm{E}+00 & 1.6 \mathrm{E}-03 \\ \text { U-238 } & 0.0 \mathrm{E}+00 & 1.6 \mathrm{E}-03 \\ \text { Vanadium } & & 0.0 \mathrm{E}+00 \\ & 0.00 & \\ \text { Ma } & & \end{array}$




\begin{tabular}{|c|c|c|}
\hline Zinc & $0.0 E+00$ & $0.0 \mathrm{E}+00$ \\
\hline Zirconium & $0.0 E+00$ & $1.3 E-03$ \\
\hline $12378 \mathrm{PeCDF}$ & $0.0 E+00$ & $0.0 \mathrm{E}+00$ \\
\hline $23478 \mathrm{PeCDF}$ & $0.0 E+00$ & $0.0 \mathrm{E}+00$ \\
\hline OCDD & $0.0 \mathrm{E}+00$ & $0.0 \mathrm{E}+00$ \\
\hline OCDF & $0.0 \mathrm{E}+00$ & $0.0 E+00$ \\
\hline $1234678 \mathrm{HpCDD}$ & $0.0 \mathrm{E}+00$ & $0.0 \mathrm{E}+00$ \\
\hline $1234678 \mathrm{HpCDF}$ & $0.0 E+00$ & $0.0 \mathrm{E}+00$ \\
\hline $123478 \mathrm{HXCDF}$ & $0.0 \mathrm{E}+00$ & $0.0 \mathrm{E}+00$ \\
\hline $12378 \mathrm{HxCDD}$ & $0.0 E+00$ & $0.0 \mathrm{E}+00$ \\
\hline $123678 \mathrm{HxCDD}$ & $0.0 E+00$ & $0.0 \mathrm{E}+00$ \\
\hline $123789 \mathrm{HxCDD}$ & $0.0 E+00$ & $0.0 \mathrm{E}+00$ \\
\hline $2378 \mathrm{TCDD}$ & $0.0 E+00$ & $0.0 E+00$ \\
\hline $2378 \mathrm{TCDF}$ & $0.0 E+00$ & $0.0 E+00$ \\
\hline Hexachloroethene & $8.8 E-03$ & $0.0 E+00$ \\
\hline 124Trichlorb & $0.0 E+00$ & $0.0 \mathrm{E}+00$ \\
\hline Molybdenum & $0.0 \mathrm{E}+00$ & $0.0 \mathrm{E}+00$ \\
\hline Strontium & $0.00 \mathrm{E}+00$ & $0.0 E+00$ \\
\hline Acenaphthylene & $0.0 \mathrm{E}+00$ & $0.0 E+00$ \\
\hline benzidine & $0.0 E+00$ & $0.0 \mathrm{E}+00$ \\
\hline Chlordane & $0.0 E+00$ & $0.0 \mathrm{E}+00$ \\
\hline Cumene & $0.0 E+00$ & $0.0 E+00$ \\
\hline MethylPropylB & $0.0 E+00$ & $0.0 E+00$ \\
\hline 124 TriMethylB & $0.0 E+00$ & $0.0 E+00$ \\
\hline 135TriMethylB & $0.0 E+00$ & $0.0 E+00$ \\
\hline MethylethylB & $0.0 E+00$ & $0.0 E+00$ \\
\hline ButylB & $0.0 \mathrm{E}+00$ & $0.0 E+00$ \\
\hline 12DiMethylB & $9.3 E-05$ & $0.0 E+00$ \\
\hline PropylB & $0.0 \mathrm{E}+00$ & $0.0 \mathrm{E}+00$ \\
\hline Aldrin & $0.0 \mathrm{E}+00$ & $0.0 \mathrm{E}+00$ \\
\hline Aroclor 1016 & $0.0 \mathrm{E}+00$ & $0.0 E+00$ \\
\hline Aroclor 1221 & $0.0 E+00$ & $0.0 E+00$ \\
\hline Aroclor 1232 & $0.0 E+00$ & $4 \cdot 9 E-04$ \\
\hline $\mathrm{DDD}$ & $0.0 E+00$ & $0.0 E+00$ \\
\hline $\mathrm{DDE}$ & $0.0 E+00$ & $4.6 E-06$ \\
\hline DDT & $0.0 \mathrm{E}+00$ & $0.0 \mathrm{E}+00$ \\
\hline Alpha-BHC & $0.0 \mathrm{E}+00$ & 8. $2 E-04$ \\
\hline Beta-BHC & $0.0 E+00$ & 8. $2 E-04$ \\
\hline
\end{tabular}




$\begin{array}{lll}\text { Delta-BHC } & 0.0 \mathrm{E}+00 & 8.2 \mathrm{E}-04 \\ \text { Acenaphthylene } & 0.0 \mathrm{E}+00 & 1.6 \mathrm{E}-03 \\ \text { Benzoic Acid } & 0.0 \mathrm{E}+00 & 0.0 \mathrm{E}+00 \\ \text { Dibenzofuran } & 0.0 \mathrm{E}+00 & 0.0 \mathrm{E}+00 \\ \text { Diethylphth } & 1.1 \mathrm{E}-01 & 0.0 \mathrm{E}+00 \\ \text { 24-Dimethylphe } & 0.0 \mathrm{E}+00 & 0.0 \mathrm{E}+00 \\ \text { Dimethylphth } & 2.1 \mathrm{E}-03 & 0.0 \mathrm{E}+00 \\ \text { 2Methylnaptha } & 0.0 \mathrm{E}+00 & 0.0 \mathrm{E}+00 \\ \text { 2346Tetrachlor } & 0.0 \mathrm{E}+00 & 0.0 \mathrm{E}+00 \\ \text { Benzyl Alcohol } & 5.6 \mathrm{E}-16 & 0.0 \mathrm{E}+00 \\ \text { Acentonitrile } & 0.0 \mathrm{E}+00 & 0.0 \mathrm{E}+00 \\ \text { Acrolien } & 0.0 \mathrm{E}+00 & 0.0 \mathrm{E}+00 \\ \text { Acylonitrle } & 0.0 \mathrm{E}+00 & 0.0 \mathrm{E}+00 \\ \text { Bromodichloro } & 3.2 \mathrm{E}-16 & 0.0 \mathrm{E}+00 \\ \text { Bromoform } & 1.6 \mathrm{E}-01 & 0.0 \mathrm{E}+00 \\ \text { Bromometh } & 4.4 \mathrm{E}-17 & 0.0 \mathrm{E}+00 \\ \text { Carbondis } & 1.2 \mathrm{E}-01 & 0.0 \mathrm{E}+00 \\ \text { Chlorometh } & 5.7 \mathrm{E}-17 & 0.0 \mathrm{E}+00 \\ \text { 0-ChloroTu } & 0.0 \mathrm{E}+00 & 0.0 \mathrm{E}+00 \\ \text { Cumene } & 0.0 \mathrm{E}+00 & 0.0 \mathrm{E}+00 \\ \text { Dibromochloro } & 2.1 \mathrm{E}-01 & 0.0 \mathrm{E}+00 \\ \text { Dichlorodiflo } & 2.9 \mathrm{E}-02 & 0.0 \mathrm{E}+00 \\ \text { 12cisDichloro } & 0.0 \mathrm{E}+00 & 0.0 \mathrm{E}+00 \\ \text { 12transDichl } & 4.7 \mathrm{E}-03 & 0.0 \mathrm{E}+00 \\ \text { 12Dichlprop } & 2.4 \mathrm{E}-01 & 0.0 \mathrm{E}+00 \\ \text { Ethylbenz } & 1.7 \mathrm{E}-02 & 0.0 \mathrm{E}+00 \\ \text { Nhexane } & 9.7 \mathrm{E}-04 & 9.7 \mathrm{E}-04 \\ \text { MethylIso } & 0.0 \mathrm{E}+00 & 0.0 \mathrm{E}+00 \\ \text { MethChloride } & 2.6 \mathrm{E}-20 & 0.0 \mathrm{E}+00 \\ \text { PropGlycol } & 0.0 \mathrm{E}+00 & 0.0 \mathrm{E}+00 \\ \text { Styrene } & 0.0 \mathrm{E}+00 & 0.0 \mathrm{E}+00 \\ \text { 1112Tetra } & 1.1 \mathrm{E}-01 & 0.0 \mathrm{E}+00 \\ \text { 1122Tetra } & 2.0 \mathrm{E}-01 & 0.0 \mathrm{E}+00 \\ \text { TriChloFlo } & 1.1 \mathrm{E}-01 & 0.0 \mathrm{E}+00 \\ \text { 123Trichlopr } & 1.8 \mathrm{E}-01 & 0.0 \mathrm{E}+00 \\ \text { Trimethbenz } & 0.0 \mathrm{E}+00 & 0.0 \mathrm{E}+00 \\ \text { 135Trimeth } & 0.0 \mathrm{E}+00 & 0.0 \mathrm{E}+00 \\ \text { oxylene } & 1.1 \mathrm{E}-02 & 0.0 \mathrm{E}+00 \\ & & \end{array}$




$\begin{array}{lll}\text { acetophenone } & 2.3 \mathrm{E}-01 & 0.0 \mathrm{E}+00 \\ \text { Ethylchlorid } & 1.8 \mathrm{E}-10 & 0.0 \mathrm{E}+00 \\ \text { 2Hexanone } & 5.9 \mathrm{E}-18 & 0.0 \mathrm{E}+00 \\ \text { Methonal } & 0.0 \mathrm{E}+00 & 0.0 \mathrm{E}+00 \\ \text { MMetacrylate } & 2.6 \mathrm{E}-20 & 0.0 \mathrm{E}+00 \\ \text { Chlorobenzene } & 4.8 \mathrm{E}-02 & 0.0 \mathrm{E}+00 \\ \text { Cyanide } & 0.0 \mathrm{E}+00 & 0.0 \mathrm{E}+00 \\ \text { Dibenzla, h] } & 0.0 \mathrm{E}+00 & 0.0 \mathrm{E}+00 \\ \text { 26Dinitrotoluene } & 3.6 \mathrm{E}-02 & 0.0 \mathrm{E}+00 \\ \text { DiNoctylphtalate } & 0.0 \mathrm{E}+00 & 0.0 \mathrm{E}+00 \\ \text { Endosulfan } & 0.0 \mathrm{E}+00 & 4.6 \mathrm{E}-05 \\ \text { 4Nitrobenzenamin } & 1.1 \mathrm{E}-09 & 1.1 \mathrm{E}-09 \\ \text { 4Nitrophenol } & 0.0 \mathrm{E}+00 & 0.0 \mathrm{E}+00 \\ \text { NNitrosodiphen } & 0.0 \mathrm{E}+00 & 0.0 \mathrm{E}+00 \\ \text { XYlene } & 0.0 \mathrm{E}+00 & 0.0 \mathrm{E}+00 \\ \text { 12Dichloro } & 0.0 \mathrm{E}+00 & 0.0 \mathrm{E}+00 \\ \text { 13Dichloro } & 0.0 \mathrm{E}+00 & 0.0 \mathrm{E}+00 \\ \text { 14Dichloro } & 0.0 \mathrm{E}+00 & 0.0 \mathrm{E}+00 \\ \text { Methylcyclo } & 1.4 \mathrm{E}-03 & 1.4 \mathrm{E}-03 \\ \text { Benzo[g,h,i] } & 0.0 \mathrm{E}+00 & 0.0 \mathrm{E}+00 \\ \text { MethylChlor } & 3.4 \mathrm{E}-17 & 0.0 \mathrm{E}+00 \\ \text { 2MethylNap } & 0.0 \mathrm{E}+00 & 0.0 \mathrm{E}+00 \\ \text { 2Nitrophenol } & 0.0 \mathrm{E}+00 & 0.0 \mathrm{E}+00 \\ \text { Aroclorl268 } & 0.0 \mathrm{E}+00 & 0.0 \mathrm{E}+00 \\ \text { EndosulfanII } & 9.3 \mathrm{E}-21 & 4.6 \mathrm{E}-05 \\ \text { Aldehyde } & 0.0 \mathrm{E}+00 & 2.6 \mathrm{E}-05 \\ \text { Ketone } & 0.0 \mathrm{E}+00 & 2.6 \mathrm{E}-05 \\ \text { Chlorobenzene } & 5.1 \mathrm{E}-02 & 0.0 \mathrm{E}+00 \\ \text { Vinyl Chloride } & 1.2 \mathrm{E}-01 & 0.0 \mathrm{E}+00 \\ \text { o-cresol } & 1.9 \mathrm{E}-01 & 0.0 \mathrm{E}+00 \\ \mathrm{~m} \text {-cresol } & 2.5 \mathrm{E}-01 & 0.0 \mathrm{E}+00 \\ \text { p-cresol } & 2.2 \mathrm{E}-01 & 0.0 \mathrm{E}+00 \\ \text { 14Dichlorobenzen } & 0.0 \mathrm{E}+00 & 0.0 \mathrm{E}+00 \\ \text { Hexachlorobenzen } & 0.0 \mathrm{E}+00 & 0.0 \mathrm{E}+00 \\ \text { Hexachlorobutadn } & 0.0 \mathrm{E}+00 & 3.3 \mathrm{E}-04 \\ \text { Hexachloroethane } & 0.0 \mathrm{E}+00 & 0.0 \mathrm{E}+00 \\ \text { Nitrobenzene } & 2.1 \mathrm{E}-01 & 0.0 \mathrm{E}+00 \\ \text { 246-Trichlorphnl } & 0.0 \mathrm{E}+00 & 0.0 \mathrm{E}+00 \\ & & \end{array}$




$\begin{array}{lll}\text { Pyridine } & 0.0 \mathrm{E}+00 & 0.0 \mathrm{E}+00 \\ 24-\mathrm{D} & 7.0 \mathrm{E}-02 & 0.0 \mathrm{E}+00 \\ 245-\mathrm{TP} \text { (Silvex) } & 2.0 \mathrm{E}-02 & 0.0 \mathrm{E}+00 \\ \text { Chlordane } & 0.0 \mathrm{E}+00 & 0.0 \mathrm{E}+00 \\ \text { Endrin } & 0.0 \mathrm{E}+00 & 2.6 \mathrm{E}-05 \\ \text { Heptachlor } & 0.0 \mathrm{E}+00 & 1.8 \mathrm{E}-05 \\ \text { Heptachlor epoxd } & 0.0 \mathrm{E}+00 & 2.2 \mathrm{E}-05 \\ \text { Lindane } & 0.0 \mathrm{E}+00 & 8.2 \mathrm{E}-04 \\ \text { Methoxychlor } & 0.0 \mathrm{E}+00 & 0.0 \mathrm{E}+00 \\ \text { Toxaphene } & 0.0 \mathrm{E}+00 & 0.0 \mathrm{E}+00 \\ \text { Lithium } & 0.0 \mathrm{E}+00 & 0.0 \mathrm{E}+00 \\ \text { l24trimethylb } & 0.0 \mathrm{E}+00 & 0.0 \mathrm{E}+00 \\ \text { lhexanol } & 5.9 \mathrm{E}-18 & 0.0 \mathrm{E}+00 \\ \text { 2hexanone } & 5.9 \mathrm{E}-18 & 0.0 \mathrm{E}+00\end{array}$

CONTAMINANT CONCENTRATIONS IN RIVER (mg/l)
TIMES
1000.
100000.

$\begin{array}{lll}\text { Acenaphthene } & 0.0 \mathrm{E}+00 & 0.0 \mathrm{E}+00 \\ \text { Acetone } & 5.2 \mathrm{E}-13 & 0.0 \mathrm{E}+00 \\ \text { Anthracene } & 0.0 \mathrm{E}+00 & 0.0 \mathrm{E}+00 \\ \text { Aluminum, } & 0.0 \mathrm{E}+00 & 0.0 \mathrm{E}+00 \\ \text { Antimony } & 0.0 \mathrm{E}+00 & 5.7 \mathrm{E}-19 \\ \text { Arochlors-1242 } & 0.0 \mathrm{E}+00 & 0.0 \mathrm{E}+00 \\ \text { Arochlors-1254 } & 0.0 \mathrm{E}+00 & 0.0 \mathrm{E}+00 \\ \text { Arochlors-1248 } & 0.0 \mathrm{E}+00 & 0.0 \mathrm{E}+00 \\ \text { Arochlors-1260 } & 0.0 \mathrm{E}+00 & 0.0 \mathrm{E}+00 \\ \text { Arsenic } & 0.0 \mathrm{E}+00 & 7.6 \mathrm{E}-02 \\ \text { Barium } & 4.0 \mathrm{E}-02 & 4.0 \mathrm{E}-02 \\ \text { Benzene } & 0.0 \mathrm{E}+00 & 0.0 \mathrm{E}+00 \\ \text { BenzoAanthracene } & 0.0 \mathrm{E}+00 & 0.0 \mathrm{E}+00 \\ \text { Beryllium } & 0.0 \mathrm{E}+00 & 0.0 \mathrm{E}+00 \\ \text { Bis2ethylhexapht } & 0.0 \mathrm{E}+00 & 0.0 \mathrm{E}+00 \\ \text { Butylbenzylphtha } & 0.0 \mathrm{E}+00 & 0.0 \mathrm{E}+00 \\ \text { Cadmium } & 0.0 \mathrm{E}+00 & 0.0 \mathrm{E}+00 \\ \text { Calcium } & 0.0 \mathrm{E}+00 & 0.0 \mathrm{E}+00\end{array}$




$\begin{array}{lll}\text { Carbazole } & 0.0 \mathrm{E}+00 & 6.4 \mathrm{E}-03 \\ \text { Carbontetchl } & 0.0 \mathrm{E}+00 & 0.0 \mathrm{E}+00 \\ \text { Chloroform } & 0.0 \mathrm{E}+00 & 0.0 \mathrm{E}+00 \\ \text { Chromium III } & 0.0 \mathrm{E}+00 & 0.0 \mathrm{E}+00 \\ \text { Chromium VI } & 0.0 \mathrm{E}+00 & 0.0 \mathrm{E}+00 \\ \text { Cobalt } & 0.0 \mathrm{E}+00 & 0.0 \mathrm{E}+00 \\ \text { Copper } & 0.0 \mathrm{E}+00 & 0.0 \mathrm{E}+00 \\ \text { Benzobflranthene } & 0.0 \mathrm{E}+00 & 0.0 \mathrm{E}+00 \\ \text { BenzoKflranthene } & 0.0 \mathrm{E}+00 & 0.0 \mathrm{E}+00 \\ \text { Benzoghiperylene } & 0.0 \mathrm{E}+00 & 0.0 \mathrm{E}+00 \\ \text { Benzo(a)pyrene } & 0.0 \mathrm{E}+00 & 0.0 \mathrm{E}+00 \\ \text { Dibenzoahanthrac } & 0.0 \mathrm{E}+00 & 0.0 \mathrm{E}+00 \\ \text { Dibenzofuran } & 5.9 \mathrm{E}+00 & 0.0 \mathrm{E}+00 \\ \text { Dieldrin } & 0.0 \mathrm{E}+00 & 6.5 \mathrm{E}-02 \\ \text { Dinbutylphthalat } & 0.0 \mathrm{E}+00 & 0.0 \mathrm{E}+00 \\ \text { Dinoctylphthalat } & 0.0 \mathrm{E}+00 & 0.0 \mathrm{E}+00 \\ \text { 24-dinitrotoluen } & 0.0 \mathrm{E}+00 & 0.0 \mathrm{E}+00 \\ \text { Fluoranthene } & 0.0 \mathrm{E}+00 & 0.0 \mathrm{E}+00 \\ \text { Flourene } & 0.0 \mathrm{E}+00 & 0.0 \mathrm{E}+00 \\ \text { 2-Hexanone } & 4.8 \mathrm{E}+00 & 0.0 \mathrm{E}+00 \\ \text { Indenoli3cdpyren } & 0.0 \mathrm{E}+00 & 0.0 \mathrm{E}+00 \\ \text { Iron } & 0.0 \mathrm{E}+00 & 0.0 \mathrm{E}+00 \\ \text { Isophorone } & 0.0 \mathrm{E}+00 & 0.0 \mathrm{E}+00 \\ \text { Lead } & 0.0 \mathrm{E}+00 & 0.0 \mathrm{E}+00 \\ \text { Lithium } & 0.0 \mathrm{E}+00 & 0.0 \mathrm{E}+00 \\ \text { Magnesium } & 0.0 \mathrm{E}+00 & 0.0 \mathrm{E}+00 \\ \text { Manganese } & 0.0 \mathrm{E}+00 & 0.0 \mathrm{E}+00 \\ \text { Mercury } & 0.0 \mathrm{E}+00 & 0.0 \mathrm{E}+00 \\ \text { Mehylenechloride } & 0.0 \mathrm{E}+00 & 0.0 \mathrm{E}+00 \\ \text { 2Methylnaphthale } & 0.0 \mathrm{E}+00 & 0.0 \mathrm{E}+00 \\ \text { Naphthalene } & 0.0 \mathrm{E}+00 & 5.7 \mathrm{E}-19 \\ \text { Nickel } & 0.0 \mathrm{E}+00 & 0.0 \mathrm{E}+00 \\ \text { NnitroNpropyl } & 4.8 \mathrm{E}+00 & 0.0 \mathrm{E}+00 \\ \text { Pentachloropheno } & 0.0 \mathrm{E}+00 & 0.0 \mathrm{E}+00 \\ \text { Phenanthrene } & 0.0 \mathrm{E}+00 & 0.0 \mathrm{E}+00 \\ \text { Phenol } & 0.1 \mathrm{E}+00 & 0.0 \mathrm{E}+00 \\ \text { Potassium } & & 0.0 \mathrm{E}+00 \\ \text { Pyrene } & 0.0 \mathrm{E}+00\end{array}$




$\begin{array}{lll}\text { Selenium } & 0.0 \mathrm{E}+00 & 0.0 \mathrm{E}+00 \\ \text { Silver } & 0.0 \mathrm{E}+00 & 0.0 \mathrm{E}+00 \\ \text { Sodium } & 0.0 \mathrm{E}+00 & 0.0 \mathrm{E}+00 \\ \text { Strontium } & 0.0 \mathrm{E}+00 & 0.0 \mathrm{E}+00 \\ \text { Tetrachloroethen } & 0.0 \mathrm{E}+00 & 0.0 \mathrm{E}+00 \\ \text { Thallium } & 0.0 \mathrm{E}+00 & 0.0 \mathrm{E}+00 \\ \text { Tin } & 0.0 \mathrm{E}+00 & 0.0 \mathrm{E}+00 \\ \text { Toluene } & 0.0 \mathrm{E}+00 & 0.0 \mathrm{E}+00 \\ \text { Trichloroethene } & 0.0 \mathrm{E}+00 & 0.0 \mathrm{E}+00 \\ \text { U-232 } & 0.0 \mathrm{E}+00 & 0.0 \mathrm{E}+00 \\ \text { U-233 } & 0.0 \mathrm{E}+00 & 3.6 \mathrm{E}-02 \\ \text { U-234 } & 0.0 \mathrm{E}+00 & 4.1 \mathrm{E}-02 \\ \text { U-235 } & 0.0 \mathrm{E}+00 & 5.5 \mathrm{E}-02 \\ \mathrm{U}-236 & 0.0 \mathrm{E}+00 & 5.5 \mathrm{E}-02 \\ \text { U-238 } & 0.0 \mathrm{E}+00 & 5.5 \mathrm{E}-02 \\ \text { Vanadium } & 0.0 \mathrm{E}+00 & 0.0 \mathrm{E}+00 \\ \text { Zinc } & 0.0 \mathrm{E}+00 & 0.0 \mathrm{E}+00 \\ \text { Zirconium } & 0.0 \mathrm{E}+00 & 4.4 \mathrm{E}-02 \\ \text { 12378PeCDF } & 0.0 \mathrm{E}+00 & 0.0 \mathrm{E}+00 \\ \text { 23478PeCDF } & 0.0 \mathrm{E}+00 & 0.0 \mathrm{E}+00 \\ \text { OCDD } & 0.0 \mathrm{E}+00 & 0.0 \mathrm{E}+00 \\ \text { OCDF } & 0.0 \mathrm{E}+00 & 0.0 \mathrm{E}+00 \\ \text { 1234678HpCDD } & 0.0 \mathrm{E}+00 & 0.0 \mathrm{E}+00 \\ \text { 1234678HpCDF } & 0.0 \mathrm{E}+00 & 0.0 \mathrm{E}+00 \\ \text { 123478HxCDF } & 0.0 \mathrm{E}+00 & 0.0 \mathrm{E}+00 \\ \text { 12378HxCDD } & 0.0 \mathrm{E}+00 & 0.0 \mathrm{E}+00 \\ \text { 123678HxCDD } & 0.0 \mathrm{E}+00 & 0.0 \mathrm{E}+00 \\ \text { 123789HxCDD } & 0.0 \mathrm{E}+00 & 0.0 \mathrm{E}+00 \\ \text { 2378TCDD } & 0.0 \mathrm{E}+00 & 0.0 \mathrm{E}+00 \\ \text { 2378TCDF } & 0.0 \mathrm{E}+00 & 0.0 \mathrm{E}+00 \\ \text { Hexachloroethene } & 3.1 \mathrm{E}-01 & 0.0 \mathrm{E}+00 \\ \text { 124Trichlorb } & 0.0 \mathrm{E}+00 & 0.0 \mathrm{E}+00 \\ \text { Molybdenum } & 0.0 \mathrm{E}+00 & 0.0 \mathrm{E}+00 \\ \text { Strontium } & 0.0 \mathrm{E}+00 & 0.0 \mathrm{E}+00 \\ \text { Acenaphthylene } & 0.0 \mathrm{E}+00 & 0.0 \mathrm{E}+00 \\ \text { benzidine } & 0.0 \mathrm{E}+00 & 0.0 \mathrm{E}+00 \\ \text { Chlordane } & 0.0 \mathrm{E}+00 & 0.0 \mathrm{E}+00 \\ \text { Cumene } & 0.0 \mathrm{E}+00 & 0.0 \mathrm{E}+00\end{array}$




$\begin{array}{lll}\text { MethylpropylB } & 0.0 \mathrm{E}+00 & 0.0 \mathrm{E}+00 \\ \text { 124TrimethylB } & 0.0 \mathrm{E}+00 & 0.0 \mathrm{E}+00 \\ \text { 135TriMethylB } & 0.0 \mathrm{E}+00 & 0.0 \mathrm{E}+00 \\ \text { MethylEthylB } & 0.0 \mathrm{E}+00 & 0.0 \mathrm{E}+00 \\ \text { ButylB } & 0.0 \mathrm{E}+00 & 0.0 \mathrm{E}+00 \\ \text { 12DiMethylB } & 3.2 \mathrm{E}-03 & 0.0 \mathrm{E}+00 \\ \text { PropylB } & 0.0 \mathrm{E}+00 & 0.0 \mathrm{E}+00 \\ \text { Aldrin } & 0.0 \mathrm{E}+00 & 0.0 \mathrm{E}+00 \\ \text { Aroclor1016 } & 0.0 \mathrm{E}+00 & 0.0 \mathrm{E}+00 \\ \text { Aroclorl221 } & 0.0 \mathrm{E}+00 & 0.0 \mathrm{E}+00 \\ \text { Aroclorl232 } & 0.0 \mathrm{E}+00 & 1.7 \mathrm{E}-02 \\ \text { DDD } & 0.0 \mathrm{E}+00 & 0.0 \mathrm{E}+00 \\ \text { DDE } & 0.0 \mathrm{E}+00 & 1.4 \mathrm{E}-04 \\ \text { DDT } & 0.0 \mathrm{E}+00 & 0.0 \mathrm{E}+00 \\ \text { Alpha-BHC } & 0.0 \mathrm{E}+00 & 2.8 \mathrm{E}-02 \\ \text { Beta-BHC } & 0.0 \mathrm{E}+00 & 2.8 \mathrm{E}-02 \\ \text { Delta-BHC } & 0.0 \mathrm{E}+00 & 2.8 \mathrm{E}-02 \\ \text { Acenaphthylene } & 0.0 \mathrm{E}+00 & 5.7 \mathrm{E}-02 \\ \text { Benzoic Acid } & 0.0 \mathrm{E}+00 & 0.0 \mathrm{E}+00 \\ \text { Dibenzofuran } & 0.0 \mathrm{E}+00 & 0.0 \mathrm{E}+00 \\ \text { Diethylphth } & 3.8 \mathrm{E}+00 & 0.0 \mathrm{E}+00 \\ \text { 24-Dimethylphe } & 0.0 \mathrm{E}+00 & 0.0 \mathrm{E}+00 \\ \text { Dimethylphth } & 7.4 \mathrm{E}-02 & 0.0 \mathrm{E}+00 \\ \text { 2Methylnaptha } & 0.0 \mathrm{E}+00 & 0.0 \mathrm{E}+00 \\ \text { 2346Tetrachlor } & 0.0 \mathrm{E}+00 & 0.0 \mathrm{E}+00 \\ \text { Benzyl Alcohol } & 2.0 \mathrm{E}-14 & 0.0 \mathrm{E}+00 \\ \text { Acentonitrile } & 0.0 \mathrm{E}+00 & 0.0 \mathrm{E}+00 \\ \text { Acrolien } & 0.0 \mathrm{E}+00 & 0.0 \mathrm{E}+00 \\ \text { Acylonitrle } & 0.0 \mathrm{E}+00 & 0.0 \mathrm{E}+00 \\ \text { Bromodichloro } & 1.1 \mathrm{E}-14 & 0.0 \mathrm{E}+00 \\ \text { Bromoform } & 5.4 \mathrm{E}+00 & 0.0 \mathrm{E}+00 \\ \text { Bromometh } & 1.5 \mathrm{E}-15 & 0.0 \mathrm{E}+00 \\ \text { CarbonDis } & 4.2 \mathrm{E}+00 & 0.0 \mathrm{E}+00 \\ \text { Chlorometh } & 2.0 \mathrm{E}-15 & 0.0 \mathrm{E}+00 \\ \text { 0-ChloroTu } & 0.0 \mathrm{E}+00 & 0.0 \mathrm{E}+00 \\ \text { Cumene } & 0.0 \mathrm{E}+00 & 0.0 \mathrm{E}+00 \\ \text { Dibromochloro } & 7.4 \mathrm{E}+00 & 0.0 \mathrm{E}+00 \\ \text { Dichlorodiflo } & 1.0 \mathrm{E}+00 & 0.0 \mathrm{E}+00\end{array}$




$\begin{array}{lll}\text { 12cisDichloro } & 0.0 \mathrm{E}+00 & 0.0 \mathrm{E}+00 \\ \text { 12transDichl } & 1.6 \mathrm{E}-01 & 0.0 \mathrm{E}+00 \\ \text { 12Dichlprop } & 8.5 \mathrm{E}+00 & 0.0 \mathrm{E}+00 \\ \text { Ethylbenz } & 6.0 \mathrm{E}-01 & 0.0 \mathrm{E}+00 \\ \text { Nhexane } & 3.4 \mathrm{E}-02 & 3.4 \mathrm{E}-02 \\ \text { MethylIso } & 0.0 \mathrm{E}+00 & 0.0 \mathrm{E}+00 \\ \text { MethChoride } & 9.0 \mathrm{E}-19 & 0.0 \mathrm{E}+00 \\ \text { PropGlycol } & 0.0 \mathrm{E}+00 & 0.0 \mathrm{E}+00 \\ \text { Styrene } & 0.0 \mathrm{E}+00 & 0.0 \mathrm{E}+00 \\ \text { 1112Tetra } & 3.8 \mathrm{E}+00 & 0.0 \mathrm{E}+00 \\ \text { 1122Tetra } & 7.0 \mathrm{E}+00 & 0.0 \mathrm{E}+00 \\ \text { TrichloFlo } & 3.9 \mathrm{E}+00 & 0.0 \mathrm{E}+00 \\ \text { 123Trichlopr } & 6.2 \mathrm{E}+00 & 0.0 \mathrm{E}+00 \\ \text { Trimethbenz } & 0.0 \mathrm{E}+00 & 0.0 \mathrm{E}+00 \\ \text { 135Trimeth } & 0.0 \mathrm{E}+00 & 0.0 \mathrm{E}+00 \\ \text { oXylene } & 3.7 \mathrm{E}-01 & 0.0 \mathrm{E}+00 \\ \text { acetophenone } & 7.9 \mathrm{E}+00 & 0.0 \mathrm{E}+00 \\ \text { Ethylchlorid } & 6.2 \mathrm{E}-09 & 0.0 \mathrm{E}+00 \\ \text { 2Hexanone } & 2.0 \mathrm{E}-16 & 0.0 \mathrm{E}+00 \\ \text { Methonal } & 0.0 \mathrm{E}+00 & 0.0 \mathrm{E}+00 \\ \text { MMetacrylate } & 9.0 \mathrm{E}-19 & 0.0 \mathrm{E}+00 \\ \text { Chlorobenzene } & 1.7 \mathrm{E}+00 & 0.0 \mathrm{E}+00 \\ \text { Cyanide } & 0.0 \mathrm{E}+00 & 0.0 \mathrm{E}+00 \\ \text { Dibenz[a, h] } & 0.0 \mathrm{E}+00 & 0.0 \mathrm{E}+00 \\ \text { 24Dinitrotoluene } & 9.6 \mathrm{E}-01 & 0.0 \mathrm{E}+00 \\ \text { 26Dinitrotoluene } & 1.3 \mathrm{E}+00 & 0.0 \mathrm{E}+00 \\ \text { DiNoctylphtalate } & 0.0 \mathrm{E}+00 & 0.0 \mathrm{E}+00 \\ \text { Endosulfan } & 0.0 \mathrm{E}+00 & 1.6 \mathrm{E}-03 \\ \text { 4Nitrobenzenamin } & 3.8 \mathrm{E}-08 & 3.8 \mathrm{E}-08 \\ \text { 4Nitrophenol } & 0.0 \mathrm{E}+00 & 0.0 \mathrm{E}+00 \\ \text { NNitrosodiphen } & 0.0 \mathrm{E}+00 & 0.0 \mathrm{E}+00 \\ \text { Xylene } & 0.0 \mathrm{E}+00 & 0.0 \mathrm{E}+00 \\ \text { 12Dichloro } & 0.0 \mathrm{E}+00 & 0.0 \mathrm{E}+00 \\ \text { 13Dichloro } & 0.0 \mathrm{E}+00 & 0.0 \mathrm{E}+00 \\ \text { 14Dichloro } & 0.0 \mathrm{E}+00 & 0.0 \mathrm{E}+00 \\ \text { Methylcyclo } & 5.0 \mathrm{E}-02 & 5.0 \mathrm{E}+02 \\ \text { Benzo[g,h, i] } & 0.0 \mathrm{E}+00 & 0.0 \mathrm{E}+00 \\ \text { Methylchlor } & 1.2 \mathrm{E}-15 & 0.0 \mathrm{E}+00 \\ & & \\ & & \end{array}$




\begin{tabular}{|c|c|c|}
\hline 2MethylNap & $0.0 \mathrm{E}+00$ & $0.0 \mathrm{E}+00$ \\
\hline 2Nitrophenol & $0.0 \mathrm{E}+00$ & $0.0 \mathrm{E}+00$ \\
\hline Aroclor 1268 & $0.0 \mathrm{E}+00$ & $0.0 \mathrm{E}+00$ \\
\hline EndosulfanII & $3.2 E-19$ & $1.6 \mathrm{E}-03$ \\
\hline Aldehyde & $0.0 E+00$ & $8.9 E-04$ \\
\hline Ketone & $0.0 \mathrm{E}+00$ & $8.9 E-04$ \\
\hline Chlorobenzene & $1.8 \mathrm{E}+00$ & $0.0 E+00$ \\
\hline Vinyl Chloride & $4.2 E+00$ & $0.0 \Xi+00$ \\
\hline o-cresol & $6.5 \mathrm{E}+00$ & $0.0 \mathrm{E}+00$ \\
\hline m-cresol & $8.7 E+00$ & $0.0 \mathrm{E}+00$ \\
\hline p-cresol & $7.8 \mathrm{E}+00$ & $0.0 \mathrm{E}+00$ \\
\hline 14Dichlorobenzen & $0.0 \mathrm{E}+00$ & $0.0 E+00$ \\
\hline Hexachlorobenzen & $0.0 \mathrm{E}+00$ & $0.0 \mathrm{E}+00$ \\
\hline Hexachlorobutadn & $0.0 E+00$ & 1. $1 \mathrm{E}-02$ \\
\hline Hexachloroethane & $0.0 \mathrm{E}+00$ & $0.0 \mathrm{E}+00$ \\
\hline Nitrobenzene & $7.4 \mathrm{E}+00$ & $0.0 \mathrm{E}+00$ \\
\hline 246-Trichlorphnl & $0.0 E+00$ & $0.0 \mathrm{E}+00$ \\
\hline Pyridine & $0.0 \mathrm{E}+00$ & $0.0 \mathrm{E}+00$ \\
\hline $24-D$ & $2.4 E+00$ & $0.0 \mathrm{E}+00$ \\
\hline 245-TP (Silvex) & $7.1 \mathrm{E}-01$ & $0.0 \mathrm{E}+00$ \\
\hline Chlordane & $0.0 \mathrm{E}+00$ & $0.0 \mathrm{E}+00$ \\
\hline Endrin & $0.0 E+00$ & $8.9 E-04$ \\
\hline Heptachlor & $0.0 \mathrm{E}+00$ & $6.4 E-04$ \\
\hline Heptachlor epoxd & $0.0 \mathrm{E}+00$ & $7.1 E-04$ \\
\hline Lindane & $0.0 E+00$ & $2.8 E-02$ \\
\hline Methoxychlor & $0.0 E+00$ & $0.0 E+00$ \\
\hline Toxaphene & $0.0 E+00$ & $0.0 \mathrm{E}+00$ \\
\hline Lithium & $0.0 E+00$ & $0.0 \mathrm{E}+00$ \\
\hline 124 trimethylb & $0.0 E+00$ & $0.0 \mathrm{E}+00$ \\
\hline 1hexanol & $5.9 E-18$ & $0.0 \mathrm{E}+00$ \\
\hline 2 hexanone & 5. $9 E-18$ & $0.0 \mathrm{E}+00$ \\
\hline butylbenzene & $0.0 \mathrm{E}+00$ & $0.0 \mathrm{E}+00$ \\
\hline
\end{tabular}

